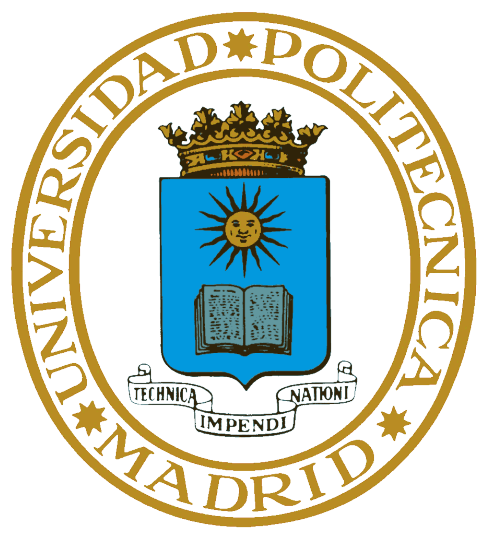

ESCUELA TÉCNICA SUPERIOR DE INGENIEROS INFORMÁTICOS

\title{
A multi-language and multi-platform framework for resource consumption analysis and its application to energy-efficient software development
}

\author{
PHD THESIS
}

Umer Liqat 



\section{A multi-language and multi-platform framework for resource consumption analysis and its application to energy-efficient software development}

SUBMITTED IN PARTIAL FULFILLMENT OF THE REQUIREMENTS FOR THE DEGREE OF:

Doctor of Philosophy in Computer Science

July, 2018

\section{Author: Umer Liqat}

M.Sc. Computational Logic (double degree),

Dresden University of Technology and Universidad Politécnica de Madrid

Advisor:

Prof. Pedro López García

Thesis Committee:

Prof. Manuel de Hermenegildo Salinas Universidad Politécnica de Madrid (Chair)

Prof. Julio Mariño Carballo (Secretary) Universidad Politécnica de Madrid

Prof. Ricardo Peña Marí

Universidad Complutense de Madrid

Prof. Antonio Fernández Anta

Instituto IMDEA Redes

Prof. María del Mar Gallardo Melgarejo

Universidad de Málaga 



\section{Abstract}

Reducing and controlling the energy consumption and the environmental impact of computing technologies have become a challenging problem worldwide. It is a significant issue in systems ranging from small Internet of Things devices, sensors, smart watches, smart phones and portable/implantable medical devices, to large data centers and high-performance computing systems. In spite of the recent rapid advances in energy-efficient hardware, including battery and energy harvesting technology, it is software that controls the hardware, so that the greatest savings are expected from developing software applications that perform a better management of the energy-saving features and resources provided by the hardware. For a given system, the potential for energy savings is likely to be much greater at the higher levels of abstraction in the system stack. Thus, promoting energy efficiency to a first class software design goal has become an important research challenge. This thesis addresses this challenge and provides tools and techniques for energy-aware software development.

First, the thesis focuses on the development of techniques for the estimation of lower and upper bounds on the energy consumed by software applications, as well as the verification that such applications meet some energy budgets (given as specifications). Addressing a challenging objective, such techniques perform the estimations statically (i.e., at compile-time, without running the program with concrete data), and give such information in the form of functions on the input data sizes of programs (and possibly other parameters, such as clock frequency, voltage, etc.). The proposed approach performs a combination of techniques for static program analysis with techniques for modeling hardware platforms. The energy models are used to represent the effect, regarding energy consumption on the hardware, of running basic software elements (e.g., low-level assembly instructions or blocks). The static analysis propagates such information through code segments, conditionals, loops, recursions, etc., in order to infer the energy consumption of the whole program.

One of the main contributions of the thesis is a multi-language general resource consumption analysis, and a specialization that infers both lower- and upper-bound energy functions at two levels, the Instruction Set Architecture (ISA) and the intermediate code (LLVM IR) levels, and reflects them upwards to the higher source code level. To achieve this, we develop translations from both ISA and the LLVM IR to an intermediate representation (Horn clauses) on which the analysis operates on. Another contribution is the experimental assessment of such analysis, which provides insights into the trade-off of accuracy versus analyzability at these levels, and concludes that the LLVM IR level analysis is a good compromise: it is reasonably powerful and accurate (less than $6.4 \%$ average deviation vs. hardware measurements).

The analysis estimates safe lower and upper bounds on the use of resources that are independent from the hardware, such as execution steps. However, in order to infer safe bounds for hardware-dependent resources, such as energy, the analysis needs to be fed with energy models that provide safe bounds too. To this end, the thesis proposes a novel modeling approach that consists in dividing a program into basic 
(branchless) blocks, establishing the maximal (resp. minimal) energy consumption for each block using an evolutionary algorithm. Then, such block-level model is used by the static analysis to infer tight energy bounds that are practical for energy verification and optimization applications. The approach has been tested on XC programs running on XMOS chips, but it is general enough to be applied to any microprocessor and programming language. The experimental results show that the bounds obtained by our prototype tool can be tight while remaining on the safe side of actual energy consumption in practice.

Traditional static resource analyses estimate the total resource usage of a call to a program. The thesis presents a novel resource analysis whose aim is instead the static profiling of accumulated cost, i.e., to discover, for selected parts of the program (named cost centers), bounds on the resource usage accumulated in each of those parts, which express how the total cost of a call to the main program is distributed among the different cost centers. Traditional resource analyses are parametric in the sense that the results can be functions on input data sizes. Our static profiling is also parametric, i.e., our accumulated cost estimates are also parameterized by input data sizes of the main program. Our proposal is based on the concept of cost centers and a program transformation that instruments programs to compute accumulated costs for each cost center. A size analysis is then applied to the transformed program to infer functions that return bounds on accumulated costs depending on input data sizes of the main program. Accumulated cost information is much more useful to the software developer than the standard (traditional) resource usage functions, as it allows identifying the parts of a program that should be optimized first, because of their greater impact on the total cost of program executions. We also report on our implementation and integration of the proposed techniques in the CiaoPP system, and provide some experimental results.

Finally, using the energy estimations provided by the aforementioned multi-level and multi-language energy consumption analysis, different types of optimisations, both static and dynamic, can be performed at different levels of the system stack. The thesis explores some of them and makes original contributions. In particular, the thesis proposes new methods based on evolutionary algorithms to improve energy-efficient task allocation and scheduling for DVFS-enabled multicore environments. These algorithms are able to deal with task migration and preemption. For applications that allow certain levels of variability in the accuracy of their results, the thesis also proposes algorithms to exploit the trade-off between accuracy and energy consumption. 


\section{Resumen}

La reducción y el control del consumo de energía de las tecnologías de la información, así como de su impacto medioambiental, se han convertido en todo un desafío a nivel mundial. Es un problema importante en sistemas que van desde pequeños dispositivos de Internet de las Cosas, sensores, relojes y teléfonos inteligentes, dispositivos médicos portables o implantables, hasta grandes centros de cálculo y sistemas informáticos de altas prestaciones. A pesar de los rápidos avances en hardware eficiente en consumo de energía, incluyendo nuevas baterías y tecnologías de recolección de energía, es el software el que controla el hardware, por lo que se consiguen mayores ahorros de energía al desarrollar aplicaciones software que realicen una mejor gestión de las características de ahorro de energía y recursos proporcionados por el hardware. El ahorro de energía será potencialmente mucho mayor trabajando en los niveles de abstracción más altos en la pila del sistema. Por ello, existe un creciente interés en considerar la eficiencia energética como un objetivo prioritario de diseño de software, lo cual plantea importantes desafíos de investigación. Esta tesis aborda dichos desafíos, proporcionando técnicas y herramientas para el desarrollo de software consciente del consumo de energía.

En primer lugar, la tesis se centra en la estimación de cotas inferiores y superiores de la energía consumida por las aplicaciones software, mediante un enfoque que realiza una combinación de técnicas de análisis estático de programas con técnicas de modelado de plataformas hardware. Los modelos de energía se utilizan para expresar el efecto, en términos del consumo de energía en el hardware, de la ejecución de elementos software básicos, como por ejemplo, instrucciones ensamblador o bloques de bajo nivel, y hacen que el sistema desarrollado sea paramétrico respecto a la plataforma de ejecución. El análisis estático propaga dicha información (en tiempo de compilación, sin ejecutar el programa con datos concretos, sino realizando una interpretación abstracta del mismo) a través de segmentos de código, estructuras de control condicionales, bucles, recursiones, etc., para inferir el consumo de energía de todo el programa. Dicha información se obtiene en forma de funciones que dependen de los tamaños de datos de entrada de los programas y posiblemente de otros parámetros, como la frecuencia de reloj, voltaje, etc.

El marco para la estimación del consumo de recursos propuesto es muy general, multi-lenguaje y paramétrico respecto a recursos y plataformas de ejecución, y se especializa para la inferencia de funciones que calculan cotas del consumo de energía a dos niveles de abstracción, lenguaje ensamblador y código intermedio (LLVM IR), las cuales se reflejan luego a nivel de código fuente. Para ello la tesis desarrolla traducciones de estos dos niveles a una representación intermedia (cláusulas de Horn) en la que opera el análisis. Además, realiza un estudio experimental de dicho análisis, proporcionando información útil sobre el compromiso entre la precisión de las estimaciones frente a la analizabilidad a estos dos niveles, y concluye que el análisis a nivel LLVM IR es un buen compromiso: es razonablemente potente y preciso (menos de un $6.4 \%$ de desviación media con respecto a los valores reales medidos en el hardware). 
Para recursos que son independientes del hardware (como los pasos de ejecución), el análisis estima cotas inferiores y superiores seguras. Para que las cotas sean también seguras para recursos dependientes del hardware, como la energía, se necesita incorporar modelos de energía que proporcionen también cotas seguras. En este sentido, la tesis propone un enfoque de modelado novedoso que consiste en dividir un programa en bloques básicos (sin ramificaciones), estableciendo el consumo de energía máximo (o mínimo) para cada bloque usando un algoritmo evolutivo. Dicho modelo a nivel de bloques es utilizado por el análisis estático para inferir cotas del consumo de energía más ajustadas, que son prácticas para su apliación en verificación y optimización del consumo de energía. El enfoque ha sido evaluado con programas XC ejecutando en chips XMOS, pero es lo suficientemente general como para ser aplicado a cualquier microprocesador y lenguaje de programación. Los resultados experimentales muestran que, en la práctica, las cotas obtenidas por el prototipo desarrollado son precisas, a la vez que se mantienen en el lado seguro del consumo real de energía.

Tradicionalmente, los análisis de recursos estáticos estiman el uso total de los recursos consumidos por una llamada a un programa. En esta tesis se desarrolla también un novedoso análisis de recursos cuyo objetivo es, en cambio, el perfilado estático del coste acumulado, es decir, la estimación, para partes seleccionadas del programa, denominadas centros de coste, de cotas del uso de recursos acumulados en cada uno de esos centros, que indican como se distribuye el coste total entre los mismos. El perfilado estático aquí propuesto también es paramétrico, produciendo estimaciones de coste acumulado que dependen de los tamaños de datos de entrada del programa principal. El método se basa en el concepto de centros de coste y una transformación de programa que instrumenta el mismo para calcular el coste acumulado en cada uno de dichos centros. Al programa transformado se le aplica luego un análisis de tamaños y se inferen los costes acumulados en función de los tamaños de datos de entrada del programa principal. La información de coste acumulado es mucho más útil para el desarrollador de software que el coste estándar (tradicional), ya que permite identificar las partes de un programa que deben optimizarse en primer lugar, debido a su mayor impacto en el coste total de la ejecución de una llamada al mismo. La técnica propuesta también se ha implementado e integrado en el sistema CiaoPP, y se ha realizado una evaluación experimental.

Finalmente, utilizando las estimaciones del consumo de energía obtenidas mediante las técnicas mencionadas se pueden realizar diferentes tipos de optimizaciones, tanto estáticas como dinámicas, en los correspondientes niveles de la pila del sistema. La tesis explora algunas de ellas, en particular, propone nuevos métodos basados en algorítmos evolutivos para mejorar la planificación y asignación de tareas a procesadores en arquitecturas multi-núcleo que ofrecen la posibilidad de modificar la frecuencia de reloj y el voltaje (DVFS). Dichos métodos son capaces de gestionar la migración y apropiación de tareas. Para las aplicaciones que permiten ciertos niveles de variabilidad en la precisión de sus resultados, la tesis también propone algoritmos que explotan el compromiso entre la precisión y el consumo de energía. 


\section{Acknowledgments}

First and foremost I would like to thank my supervisor Pedro Lopez Garcia for a number of reasons. First, for giving me an amazing opportunity to work with him in a very exciting research project at IMDEA Software Institute. Second, for all his time and invaluable input and supervision in development of this thesis. And third, for being more of a friend than a boss and his patience and faith in me.

I would like to express my sincere gratitude to the IMDEA Software Institute and its then Director, Manuel Hermenegildo, for financially funding my Ph.D. and providing me with a great environment for work, to its researchers for an inspiring atmosphere and to its amazing support staff for making my stay at the institute extremely smooth.

I would also like to acknowledge all the sources that have funded the different research activities throughout the development of the thesis, i.e., the Spanish MINECO TIN2012-39391 StrongSoft, TIN2015-67522-C3-1-R TRACES projects, the Madrid M141047003 N-GREENS program, and in particular the Whole Systems Energy Transparency (ENTRA) project, funded by the EU 7th Framework Programme for Future and Emerging Technologies (FET).

For all the fruitful discussions and invaluable feedback on my work, I am grateful to Manuel Hermenegildo, Maximiliano Klemen, Zorana Bankovic, John Gallagher, Remy Haemmerle, Luthfi Darmawan and others. I was happy to make a lot of good friends while being in IMDEA: Maximiliano, Raul, Zorana, Irfan, Miguel, Srdjan, et al.; thanks for all the positive energy during my stay at IMDEA, for the countless discussions, jokes, drinks, lunches, laughter.

Last, but not the least, I want to thank my wife Sahrish for all the support she has given through all these years as well as my parents for their unconditional support. 

Abstract iii

Resumen $\mathbf{v}$

1 Introduction $\quad 1$

1.1 State of the Art . . . . . . . . . . . . . 5

1.2 Thesis Objectives . . . . . . . . . . . . . . . . . . . . . . 7

1.3 Main Contributions . . . . . . . . . . . . . . . 8

1.4 Thesis Organization . . . . . . . . . . . . . . . . . . . . 9

2 Multi-language Energy Consumption Analysis and Modeling Framework 11

2.1 Introduction . . . . . . . . . . . . . . . . 11

2.1.1 Hypothesis: Analysis and Modeling Trade-off . . . . . . . . . 12

2.2 Analysis and Modeling Framework Architecture . . . . . . . . . . . 14

2.3 Static Resource Analysis . . . . . . . . . . . . . . . . . . . . 17

2.3.1 CiaoPP Resource Analysis . . . . . . . . . . . . . . 17

2.4 Energy Characterization and Modeling . . . . . . . . . . . . . . 19

2.4.1 Energy Profiling Framework and Strategy . . . . . . . . . . . 19

2.4 .2 ISA-level Model . . . . . . . . . . . . . . . . . . . . 19

2.5 Analysis at the ISA level . . . . . . . . . . . . . . . 21

2.5.1 Transforming ISA Programs into Horn Clauses . . . . . . . 23

2.5.2 Instantiating the CiaoPP Analysis Framework . . . . . . . 25

2.5.3 Performing the Analysis . . . . . . . . . . . . . . 27

2.5.4 Benchmarks, Results and Evaluation . . . . . . . . . . 29

2.6 Analysis at the LLVM IR Level . . . . . . . . . . . . . . . . . . 32

2.6.1 LLVM IR to HC IR Transformation . . . . . . . . . . . . 33

2.6.2 Obtaining the Energy Consumption of LLVM IR Blocks . . . 37

2.6.3 Resource Analysis with CiaoPP . . . . . . . . . . . . 38 
2.7 Experimental Evaluation . . . . . . . . . . . . . . . . 38

2.8 Energy Consumption Verification via Static Analysis . . . . . . . . . 42

2.8 .1 Introduction . . . . . . . . . . . . . . . . 42

2.8.2 Overview of the Energy Verification Tool . . . . . . . . . . 43

2.8 .3 The Assertion Language . . . . . . . . . . . . . . 45

2.8.4 The General Resource Usage Verification Framework . . . . . 48

2.8.5 Using the Tool: Example . . . . . . . . . . . . . . . . . 49

2.9 Related Work . . . . . . . . . . . . . . . . . . . . 51

2.10 Chapter Conclusions and Future Work . . . . . . . . . . . . . . 53

3 Evolutionary Modeling of Basic Blocks for Static Analysis of Energy 55

3.1 Introduction . . . . . . . . . . . . . . . 55

3.2 Energy Consumption Verification/Certification . . . . . . . . . 58

3.3 Modeling the Energy Consumption of Blocks . . . . . . . . . . . . 58

3.3.1 Identifying the Basic Blocks to be Modeled . . . . . . . . . 58

3.3.2 Evolutionary Algorithm for finding Energy Bounds for Basic

Blocks ................... 60

3.4 Energy Consumption of the Program . . . . . . . . . . . . . 62

3.5 Experimental Evaluation . . . . . . . . . . . . . . . 63

3.5.1 The Platform Modeled . . . . . . . . . . . . . 63

3.5.2 The Measurement Harness . . . . . . . . . . . . . . 63

3.5.3 Results and Discussion . . . . . . . . . . . . . . . 64

3.6 Related Work . . . . . . . . . . . . . . . . . . . . . 68

3.7 Chapter Conclusions and Future Work . . . . . . . . . . . . . 70

4 Transformational Approach to Parametric Accumulated-Cost Static Profiling $\quad 71$

4.1 Introduction and Motivation $\ldots \ldots \ldots 71$

4.2 From Dynamic Profiling to Static Profiling . . . . . . . . . . . . . 73

4.3 The Classical Cost Relations-based Parametric Static Analysis . . . . 75

4.4 Parametric Accumulated-Cost Static Profiling . . . . . . . . . . . . 76

4.5 Inferring Accumulated Cost via Transformation . . . . . . . . . . . 79

4.5.1 The Transformation . . . . . . . . . . . . . . 79

4.5.2 Performing the Resource Usage Analysis . . . . . . . . . . . . 81

4.6 Experimental Results . . . . . . . . . . . . . . . . 82

4.7 Related Work . . . . . . . . . . . . . . . . . . . . . . . . . . 84

4.8 Chapter Conclusions . . . . . . . . . . . . . . . . . . . 85

$\begin{array}{llr}5 & \text { Energy Efficient Scheduling } & 87\end{array}$

5.1 Introduction . . . . . . . . . . . . . . . . . 87

5.2 Energy Efficient Scheduling using Evolutionary and YDS Algorithms with Faster Energy Estimations . . . . . . . . . . . . . . . 88

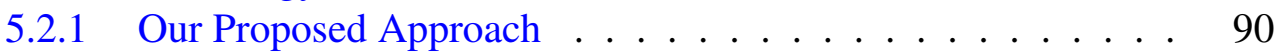

5.2.2 Experimental Evaluation . . . . . . . . . . . . . 97 
5.2.3 Section Conclusions and Future Work . . . . . . . . . . . . 100

5.3 Trade-off Accuracy vs. Energy for Energy Efficient Scheduling . . . . 102

5.3 .1 Proposed Approach . . . . . . . . . . . . . . . . 103

5.3 .2 Experimental Evaluation . . . . . . . . . . . . . 105

5.3 .3 Related Work . . . . . . . . . . . . . . . . . 110

5.3 .4 Section Conclusions . . . . . . . . . . . . . . 111

6 Conclusions and Future Work 113

$\begin{array}{ll}\text { Bibliography } & 117\end{array}$ 



\section{List of Figures}

1.1 Analysis/modeling level trade-off and potential choices. . . . . . . . . 3

1.2 Energy-aware software engineering main components. . . . . . . . 10

2.1 High-level view of the general analysis framework architecture. . . . . 15

2.2 Detailed view of the analysis/modeling layers within the analysis framework. . . . . . . . . . . . . . . . 16

2.3 Overview of test harness hardware and software structure, with a slave processor executing test kernels and a master processor collecting power samples. . . . . . . . . . . . . . . . 20

2.4 A realization of the general analysis framework using models and performing the analysis at the ISA layer. . . . . . . . . . . . . . . . . . . 22

2.5 An XC source (factorial) function. . . . . . . . . . . . . . . 22

2.6 An ISA (factorial) program (left) and its Horn-clause representation

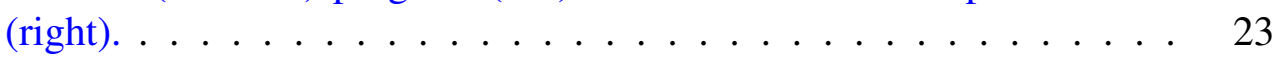

2.7 Hardware energy, estimations and relative errors for (starting top-left, moving clock-wise) fact, fibonacci, poweroftwo and power. . . . 31

2.8 An overview of the analysis at the LLVM IR level using ISA models. . 32

2.9 An XC program and its type transformation into HC IR. . . . . . . . 35

2.10 LLVM IR Array traversal example (left) and its HC IR representation (right) .......................... 36

2.11 Energy consumption verification tool using CiaoPP. . . . . . . . . 43

2.12 Graphical User Interface of the prototype with the XC biquad program. 50

3.1 Example: Basic block modifications. . . . . . . . . . . . . . 59

3.2 Example of even-odd crossover. . . . . . . . . . . . . . 61

3.3 Mutation. . . . . . . . . . . . . . . . . 61

3.4 Estimated energy upper/lower bounds vs. actual measurements. . . . . 66 
5.1 Overview of our scheduling approach. . . . . . . . . . . . 90 90

5.2 An example of (part of) a solution (i.e., individual) representation. . . 92

5.3 An example of a crossover operation. . . . . . . . . . . . . . . 93

5.4 Examples of mutation operations. . . . . . . . . . . . . . 93

5.5 Representation of an individual . . . . . . . . . . . . . . 103

5.6 Different possibilities for mutation . . . . . . . . . . . . . 105

5.7 Pareto front for Energy/Accuracy trade-off EA (Case1) . . . . . . . . 108

5.8 Pareto front for Energy/Time trade-off EA (Case 2) . . . . . . . . . . 109

5.9 Energy savings for different accuracy levels . . . . . . . . . . . . . . 109 


\section{List of Tables}

2.1 Description of benchmark functions used in experiments and their corresponding energy functions. . . . . . . . . . . . .

2.2 Actual and estimated energy consumption for the fact (N) function over a range of $N \ldots \ldots \ldots \ldots \ldots \ldots$. . . . . . . . . . . . . 30

2.3 Comparison of the accuracy of energy analyses at the LLVM IR and ISA levels. . . . . . . . . . . . . . . . . . . . . 40

2.4 LLVM IR-vs. ISA-level analysis accuracy. . . . . . . . . . . . . . 41

3.1 Upper and lower bounds accuracy. . . . . . . . . . . . . . . 64

3.2 Source of inaccuracies in findMax prediction: analysis vs. modeling. 68

3.3 Source of inaccuracies in reverse prediction: analysis vs. modeling. . 69

4.1 Experimental results. . . . . . . . . . . . . . 83

5.1 Viable $(V, f)$ pairs for XMOS chips. . . . . . . . . . . . 97

5.2 Energy functions inferred by static analysis for 3 different pairs of volt-

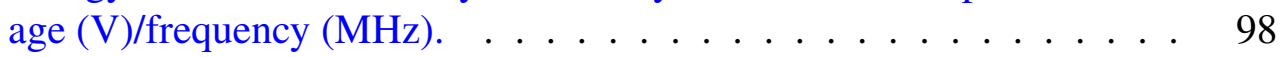

5.3 Energy savings obtained by the modified YDS vs. the original YDS (\%). 99

5.4 EA vs. improved YDS in different scenarios. . . . . . . . . . . 101

5.5 Viable $(V, f)$ pairs for XMOS chips. . . . . . . . . . . 106

5.6 Energy functions for 3 different pairs of voltage $(\mathrm{V}) /$ frequency $(\mathrm{F}$, in $\mathrm{MHz}) \ldots \ldots \ldots \ldots \ldots$. . . . . . . . . . . . . 107

5.7 Obtained savings with different levels of minimal acceptable accuracy. 108

5.8 Result of an experiment: tasks whose final number of loop iterations has been changed. . . . . . . . . . . . . . . . 110 



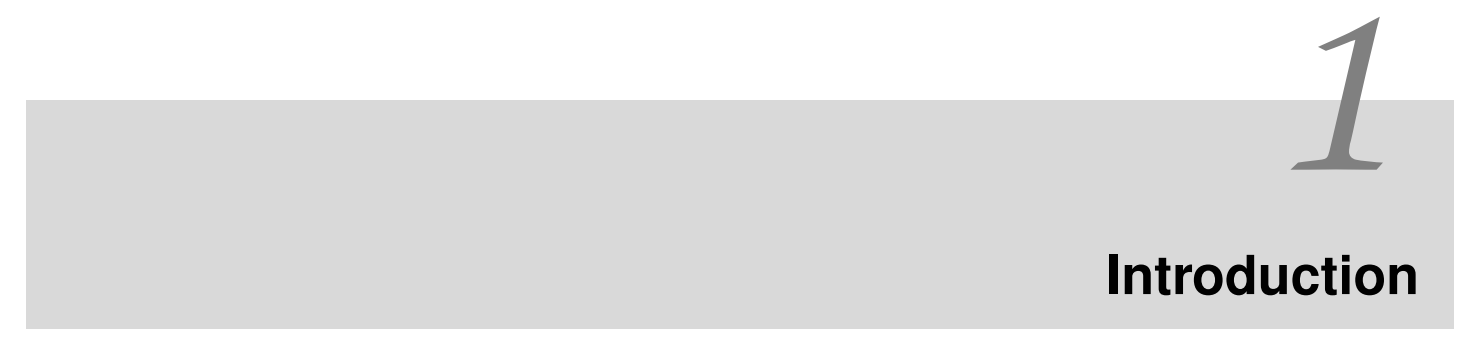

The execution of software consumes resources, such as time, memory or energy. In particular, reducing and controlling the energy consumption and the environmental impact of computing technologies have become a major worldwide concern. It is an important issue in high-performance computing, distributed applications, and data centers. There is also increased demand for small complex computing systems which have to operate on batteries, such as implantable/portable medical devices, mobile phones, Internet of Things devices, sensors or smart watches. Despite advances in powerefficient hardware, including battery and energy harvesting technology, more energy savings can be achieved by improving the way current software technologies make use of such hardware. An estimate from Intel [29] is that energy-efficient software can realize savings of a factor of three to five beyond what can be achieved through energy efficient hardware. Hence the greatest savings are expected from developing software applications that perform a better management of the energy-saving features and resources provided by the hardware. This gives rise to the important research challenge of promoting energy efficiency to a first class software design goal, and making real the vision of energy-aware software development. This thesis addresses this challenge, providing tools and techniques for energy-aware software development.

Energy transparency and the role of energy modeling and analysis: in order for the software developer to determine the effect, in terms of energy consumption, of a high-level software segment (e.g., a source code procedure), the energy consumption information at the machine level needs to be leveraged up to the higher-level in the system stack. Hence, achieving energy transparency through the system layers. This is in contrast to the modern software engineering principles where the goal is to abstract away from machine-level details by introducing different layers in the system stack (e.g., high-level languages, abstract data types, layers of interpretation/compilation, etc.) in order to achieve better understanding and reusability of the software. More concretely, this thesis contributes to the two main cornerstones for energy transparency and, hence, energy-aware software development: modeling and analysis techniques. Energy modeling deals with constructing representations (models) of how energy is consumed during the computation of basic elements of a program (e.g., assembly in- 
structions), whereas energy analysis uses these models, propagating the information they provide, to estimate the energy consumption of the whole program, as well as individual higher-level segments (e.g., procedures). Addressing a challenging objective, such (automatic) analysis techniques perform the estimations statically (i.e., at compile-time, without running the program with concrete data), and give such information in the form of functions on the input data sizes of programs (and possibly other parameters, such as clock frequency, voltage, etc.). Previous work on resource analysis, mainly for inferring asymptotic time complexity bounds, goes back to the 1970s. In this thesis, we adapt and extend existing techniques for inferring other resources, in particular energy.

Platform dependent vs. platform independent resources: our notion of the platform where the software is executed includes the hardware, the operating system, and the running environment in general. We can classify resources as platform dependent or platform independent. For example, energy or execution time are platform-dependent resources, since they depend on characteristics such as clock frequency, voltage, etc., whereas execution steps or bits-sent over a socket are platform-independent resources, since they can be derived by considering the program only, independently of what platform it is executed on. In other words, the program resource usage is the same in all platforms. The problem of automatic resource consumption analysis is rather more complex for the resources that are platform dependent compared to the resources that are platform independent. In order to infer platform-dependent resource consumption, the analysis needs to be fed with information that models the effect, in terms of resource usage, of running basic elements of the program (such as assembly, LLVM IR or bytecode instructions, or blocks of instructions) on a given platform. The closer (on the levels of the system stack) those basic elements are from the hardware, the easier it is to produce more accurate models. Examples of energy models are [54], at the Java bytecode level, or [51], at the assembly level. The analysis then mimics the program semantics (e.g., by using abstract interpretation techniques), and propagates the basic information provided by the lower-level resource models through code segments, conditionals, loops, recursions, etc., in order to infer resource usage information (e.g., energy consumption functions) for higher-level entities such as procedures, functions, or loops in the program.

Multi-language energy consumption analysis: in order to support different programming languages and different program representations at different levels of compilation, e.g., LLVM IR, Instruction Set Architecture (ISA) or bytecode, in the same analysis framework, we differentiate between the input language (which can be source code, LLVM IR, ISA or any other language) and the intermediate semantic program representation that the resource analysis actually operates on. The latter is a series of connected code blocks, represented by Horn Clauses, that we refer to as "HC IR". A transformation from each input language into the HC IR can be performed and passed to a resource analyzer, as illustrated in Figure 1.1. In particular, in this thesis we have developed transformations from both ISA and LLVM IR to HC IR. 
Analysis/modeling level trade-off: the fact that the analysis and modeling can be performed at different levels of the software stack, e.g., at the source code, LLVM IR or ISA levels, gives rise to our hypothesis that the choice of level will affect the accuracy of the energy models and the precision of the analysis in opposite ways: energy models at lower levels (e.g., at the ISA level) will be more precise than at higher levels (e.g., source code), since the closer to the hardware, the easier it is to determine the effect of the execution on the hardware. However at lower levels, more program structure and data type/shape information is lost due to lower-level representations, and we expect a corresponding loss of analysis accuracy. In this sense, we study such hypothesis and perform experiments to shed light on the existence of the trade-off of precision versus analyzability mentioned above. This hypothesis about the analysis/modeling level trade-off (and potential choices) is illustrated in Figure 1.1. In this thesis, we have explored different points in this space of combinations of analysis and modeling.

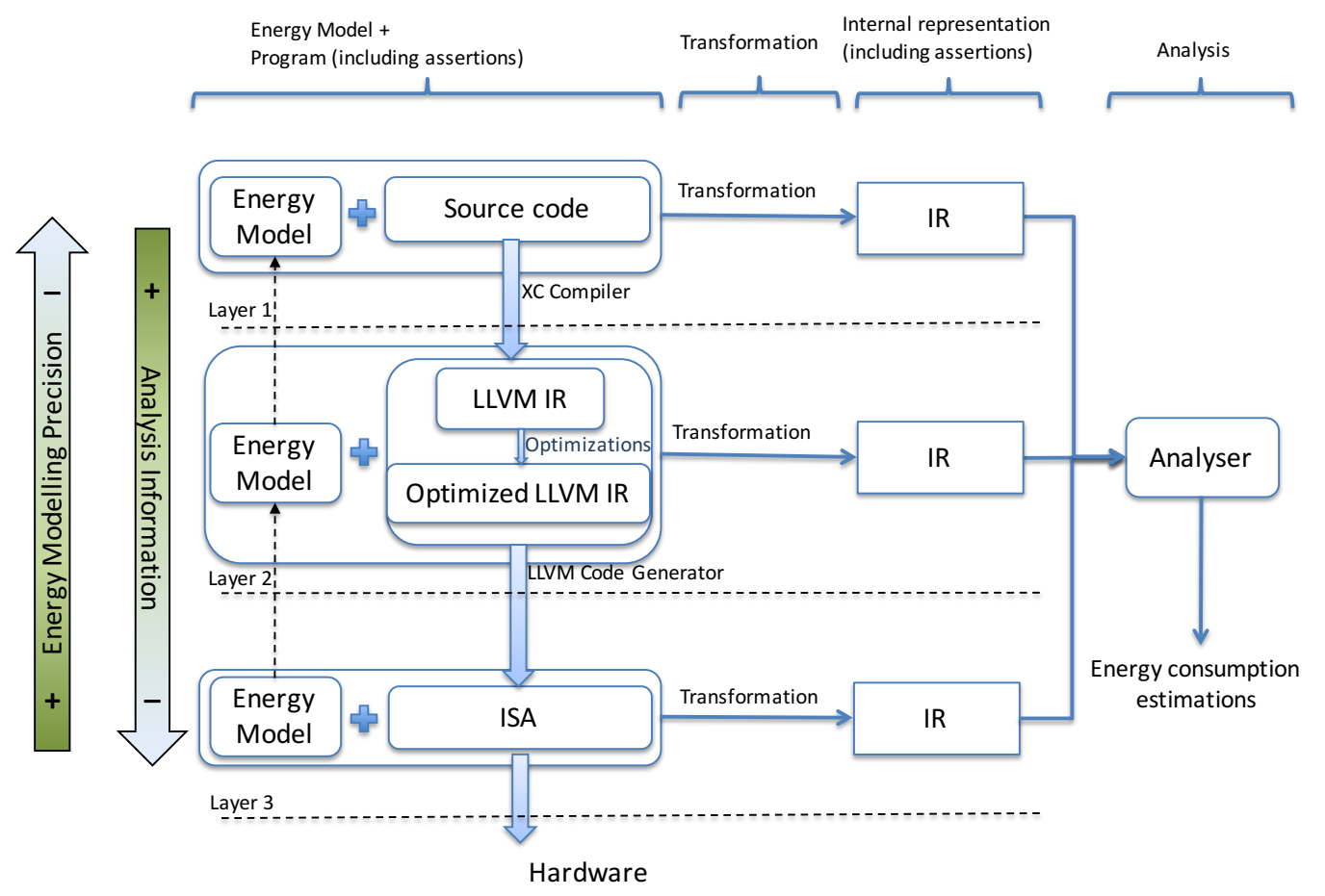

Figure 1.1: Analysis/modeling level trade-off and potential choices.

Trading-off safety and accuracy of energy estimations: clearly, the accuracy of the bounds on the energy estimations inferred by analysis depends on the nature and accuracy of the low-level models. Existing energy models such as $[54,51]$ provide average energy consumption values or functions, which are not really suitable for upper- or lower-bounds analysis. Furthermore, trying to obtain instruction-level models that provide strict safe energy bounds would compromise their accuracy with overly conservative bounds. Although when fed with such models the analysis would infer high-level energy consumption functions providing strictly safe bounds, these bounds 
would not be useful in general because of their large inaccuracy. For this reason, the analysis could use instead the already mentioned instruction level average energy models $[54,51]$. However, this would mean that the energy functions inferred for the whole program will not be strict bounds, but rather approximations of the actual bounds, and could possibly be below or above. With this safety/accuracy trade-off in mind, we propose an approach that combines a best effort modeling with the safe static analysis, to infer accurate bounds that are on the safe side in most cases, in order to be practical for verification applications, in addition to energy optimization.

Static profiling: we also investigate an extension of the static resource analysis problem which, although based on the same essential techniques, has a different range of applications. Rather than estimating the total resource usage of a call to a program, we wish to perform a static profiling of it. This means that we intend to discover, for selected parts of the program, named cost centers, bounds on such resource usage that can be attributed to each of these cost centers. We refer to such bounds as the accumulated costs on each cost center, and express how the total cost of a call to the main program is distributed among such cost centers. As before, the estimates will be parameterised by input sizes. However, these input sizes will be of the main procedure/function, unlike the input sizes of the selected parts, as in the traditional resource analysis. Our proposal is based on a program transformation that instruments programs to compute accumulated costs for each cost center. A size analysis is then applied to the transformed program to infer functions that return bounds on accumulated costs depending on input data sizes of the main program. Accumulated cost information is much more useful to the software developer than the standard (traditional) resource usage functions, as it allows identifying the parts of a program that are the most resource critical, and should be optimized first, because of their greater impact on the total cost of program executions.

Energy optimisations using estimations: different types of optimisations at different levels of the software stack can be performed by taking advantage of the energy estimations provided by the multi-language energy consumption analysis already discussed. Both static and dynamic energy optimisations are enabled by such estimations, which have been investigated in this thesis. In particular new techniques based on Evolutionary Algorithms (EAs) have been proposed to improve energy-efficient task allocation and scheduling for DVFS (Dynamic Voltage and Frequency Scalling) enabled multicore environments. Energy efficient scheduling and allocation in multicore environments is a well-known $N P$-hard problem. Nevertheless, approximated solutions can be efficiently found by heuristic algorithms, such as EAs. However, these algorithms have some drawbacks that hinder their applicability: typically they are very slow, and if the space of the feasible solutions is too restricted, they often fail to provide a viable solution. The thesis propose an approach that overcomes these issues. The approach is based on a custom EA that is fed with predicted information provided by the static analysis about the energy consumed by tasks. This solves the time inefficiency problem. In addition, when this algorithm fails to produce a feasible solution, we resort to a modification of the well-known YDS algorithm that we have performed, well adapted 
to the multicore environment and to the situations when the static power becomes the predominant part. This way, we propose a combined approach that produces an energy efficient scheduling in a reasonable time, and always finds a viable solution. For applications that allow certain levels of variability in the accuracy of their computations, the trade-off between quality of service (QoS) and energy is investigated as well. In particular, this is performed by applying loop perforation techniques [89]. The experiments conducted on a case study in different scenarios show that the new scheduler enhanced with such trade-off capability improves the previous one, achieving significant energy savings (31\% on average) for acceptable levels of accuracy loss. Other optimisations include the use of energy analysis to choose software parameters in order to transform programs to ensure that an energy target is met while minimizing the loss in quality of service. These approaches have been tested on multicore XMOS chips, but they can easily be adapted to other multicore environments as well.

\subsection{State of the Art}

Few papers can be found in the literature focusing on the static analysis of energy consumption. A similar approach was proposed for upper-bound energy analysis of Java bytecode programs in [75], which is an application of the analysis of the Java bytecode (object oriented) programs proposed in $[67,76]$ and the general resource analysis in [77]. In this approach the Jimple (a typed three-address code) representation of Java bytecode was transformed into Horn Clauses, and a simple energy model at the Java bytecode level [54] was used. However, this work did not compare the results with actual, measured energy consumption. In this approach as well as ours, instantiations for energy consumption of general resource analyzers are used, namely [77] in [75, 76], and [87] in our approach described in Chapter 2. Such resource analyzers are based on setting up and solving recurrence equations, an approach proposed by Wegbreit [96] that has been developed significantly in subsequent work [84, 26, 27, 92, 77, 3, 87]. Other approaches to static analysis based on the transformation of the analyzed code into another (intermediate) representation have been proposed for analyzing low-level languages [39] and Java (by means of a transformation into Java bytecode) [4]. In [4], cost relations are inferred directly for these bytecode programs, whereas in $[67,76]$ the bytecode is first transformed into Horn Clauses. The general resource analyzer in [77] was also instantiated in [68] for the estimation of execution times of logic programs running on a bytecode-based abstract machine. The approach used timing models at the bytecode instruction level, for each particular platform, and program-specific mappings to lift such models up to the Horn Clause level, at which the analysis was performed. The timing model was automatically produced in a one-time, programindependent profiling stage by using a set of synthetic calibration programs and setting up a system of linear equations. There exist other approaches to cost analysis such as those using dependent types [44], SMT solvers [6], or size change abstraction [106].

A number of static analyses are also aimed at worst case execution time (WCET), usually for imperative languages in different application domains (see e.g., [97] and 
its references). The worst-case analysis presented in [47], which is not based on recurrence equation solving, distinguishes instruction-specific (not proportional to time, but to data) from pipeline-specific (roughly proportional to time) energy consumption. However, in contrast to our approach and the one in [68], these worst case analysis methods do not infer cost functions on input data sizes but rather absolute maximum values, and they generally require the manual annotation of loops to express an upperbound on the number of iterations. An alternative approach to WCET was presented in [43]. It is based on the idea of amortisation, which allows to infer more accurate yet safe upper bounds by averaging the worst execution time of operations over time. It was applied to a functional language, but the approach is in principle generally applicable. A timing analysis based on game-theoretic learning was presented in [88]. The approach combines static analysis to find a set of basic paths which are then tested. In principle, such approach could be adapted to infer energy usage. Its main advantage is that this analysis can infer distributions on time, not only average values.

Static profiling in the context of Worst Case Execution Time (WCET) Analysis of real-time programs is presented in [15]. It proposes an approach to computing worstcase timing information for all code parts of a program using a complementary metric, called criticality. Every statement of a real-time program is assigned a criticality value, expressing how critical the respective code is for the global WCET. Our approach is not limited to WCET, since it is able to obtain results for a general class of user-defined resources. Furthermore, our inferred metrics are parametric on the input data sizes of the main program, in contrast to the criticality metric, which is a numeric value in the range $[0,1]$. In addition, our approach is modular and compositional, able to compute accumulated costs with respect to calls originating from different procedures of the program, and not only the main program entry point. In [13] the authors present static profiling techniques to estimate the execution likelihood and frequency of program points in order to assess whether the cost of certain compile-time optimizations would pay off. To this end, they explore the use of some static analysis techniques for predicting the result of conditional branches, such as assuming a uniform distribution over all branches, making heuristic based predictions, and performing value range propagation. In this context, our approach can be used to infer bounds on the number of times a certain program point will be called from a given entry point, as functions on input data sizes, in contrast with a single value representing the execution likelihood or frequency.

In the context of energy efficient scheduling, a great number of publications [32] try to present it as a mixed integer linear optimisation problem, which can be solved using mixed integer linear programming or using a heuristic approach. However, these algorithms become impractical or fail to deliver a solution as the problem size grows. Since DVFS can provide significant energy savings, its optimal usage has been extensively studied. Some examples divide scheduling and allocation into two separate steps, such as the one given in [81], wherein the first step the allocation problem is solved using Linear Programming, while in the second one the scheduling problem is solved for separate processors using Bin Packing. Another approach [18] solves the 
scheduling problem using a Genetic Algorithms (GAs) that integrates DVFS in the fitness function. However, such a division of the problem reduces the search space, since it becomes limited by the optimal solution of the first part of the problem, which does not always correspond to the global optimum. Our approach solves the scheduling and allocation problem at the same time while accounting for DVFS. In [52] scheduling, allocation, and power management are combined in one process, however, it only deals with voltage scaling.

An approach to the problem of optimal scheduling and allocation in multiprocessor systems that allow DVFS, presented in [70], aims to minimize both energy and makespan as a bi-objective problem. The same problem is solved in another work [71], but using the island model of parallel GA populations. Another approach [53] treats the problem from two opposite points of view: in the first one, it optimizes the energy given the scheduler length, while in the other one it optimizes the scheduling length given the energy bound. However, none of the solutions include the possibility of two levels of parallelism as in our work, where each processor can have a number of different threads executing in parallel. Moreover, as far as we know, none of the existing approaches introduce the possibility of task migration. Furthermore, they do not deal with the issue that the EAs cannot always find a viable solution, while we introduce an additional stage implemented as a modified YDS algorithm, which can always find a viable solution. Finally, using static analysis for estimating energy consumption to guide the EA is a relevant novelty of our methodology, which, as our experimental results show, achieves a significant speed-up and provides evidence about the practical applicability of such methodology.

Regarding the particular case of energy efficient scheduling where trade-off between QoS and energy is enabled, the techniques in the existing literature that include QoS as an objective in scheduling are mainly designed for Grid or Cloud Computing environments where QoS is measured as either execution time, cost, etc., which has to be provided according to the signed Service Level Agreement (SLA) between the provider and the customer [104, 103, 105]. Multiobjective genetic algorithms were used in [105] to minimize cost and execution time since they can be in conflict. A similar approach is presented in [104]. In [103] the authors try to minimize energy and maximize QoS at the same time in a Cloud Computing environment. The multiobjective optimisation problem is solved using particle swarm optimisation. However, as far as we know, none of the approaches in the literature propose to trade off QoS (accuracy in our case) with energy or performance in a scheduling problem by transforming the code, in our case by using loop perforation [89].

\subsection{Thesis Objectives}

The general objective of the thesis is the development of techniques and tools for energy-aware software engineering, promoting energy efficiency into a first-class design goal. In order to contribute to this general objective, we will focus on the following particular objectives: 
- Developing combined analysis/modeling techniques and tools for estimating the energy consumed by programs, as well as developing energy optimizations techniques that exploit such information.

- Produce the kind of information that can be effectively used for:

- Helping developers make energy-related design decisions (e.g., hotspot detection).

- Verifying that programs meet energy consumption specifications.

- Performing automatic energy optimizations, in particular, by developing task scheduling and allocation algorithms for (DVFS-enabled) multicore environments to improve energy efficiency, and exploiting the trade-off between quality of service (QoS) and energy consumption.

- Make such tools general enough to be applied in a broad range of real environments, i.e., with different programming languages and architectures.

\subsection{Main Contributions}

The main contributions of the thesis, that achieve the objectives above, are enumerated and summarized below, along with the international fora where the thesis results have been presented and/or published. Such international fora include first class conferences and JCR indexed journals. The thesis publications are co-authored with other researchers, and in all of them, the contribution of the candidate has been relevant.

- Development of a transformation based multi-language resource consumption analysis, specialized for inferring both lower and upper bounds on program energy consumption statically (i.e., at compile-time, without running the program with concrete data), that gives such information in the form of functions on the input data sizes of programs (and possibly other parameters, such as clock frequency, voltage, etc.). Such estimations are inferred at two levels, the instruction set architecture (ISA) and the intermediate code (LLVM IR) levels, and reflected upwards to the higher source code level. In particular, this required the development of a transformation from ISA and LLVM IR representations into HC IR, which enables a multi-level/multi-language analysis. It also allows a developer to study the trade-off of precision versus analyzability at different levels of the system stack, as it is done in the thesis.

The results are published in the proceedings of the 23rd International Symposium on Logic-Based Program Synthesis and Transformation (LOPSTR'13) [62] and in the proceedings of the Foundational and Practical Aspects of Resource Analysis (FOPARA'15) [61], and a summary of them in the JCR indexed journal of Microprocessors and Microsystems (MICPRO'16) [28], coauthored with other partnering institutions/companies of the ENTRA project. 
- A novel energy modeling technique that approximates both upper and lower bounds on the energy of "branchless" blocks of instructions, in order to take into account the inter-instruction switching costs within a block using an Evolutionary Algorithm. Then, such block-level energy model is fed into our static analysis, which takes into account the program control-flow, and infers energy information for the whole program and its procedures. This approach is general enough to be applied to any microprocessor and programming language, hence making our overall framework multi-platform and multi-language.

The results were presented in the International Workshop on High Performance Energy Efficient Embedded Systems (HIP3ES'16) [58] and presented and accepted for publication in the post-conference proceedings of the 27th International Symposium on Logic-Based Program Synthesis and Transformation (LOPSTR'17) [60].

- A novel resource analysis whose aim, compared to traditional static resource analysis, is the static profiling of accumulated cost, i.e., to discover, for selected parts of the program, bounds on the resource usage accumulated in each of those parts. Such information is much more useful to the software developer than the traditional resource usage functions, as it allows identifying the parts of a program that should be optimized first, because of their greater impact on the total cost of program executions.

The results are published in the proceedings of the Thirteenth International Symposium on Functional and Logic Programming (FLOPS'16) [38].

- An energy-efficient scheduling and task allocation algorithm for multicore systems based on an Evolutionary Algorithm that uses static analysis to estimate the energy consumption of tasks to schedule. The resulting schedules are shown to obtain significant energy savings.

The results are published in the JCR indexed Journal of Multiple-Valued Logic and Soft Computing (JMVLSC'16) [9] and in the 11th International Conference on Artificial Intelligence Applications and Innovations (AIAI'15) [7].

- An energy-efficient scheduling algorithm to trade off accuracy and energy, for applications that allow certain levels of variability in the accuracy of their computations, and have to meet energy budgets.

The results are published in the JCR indexed journal Logic Journal of the IGPL (IGPL'17) [59] and in the 10th International Conference on Hybrid Artificial Intelligence Systems (HAIS'15) [8].

\subsection{Thesis Organization}

In the rest of the thesis, each chapter describes one (or a combination) of the papers mentioned in the contributions. Each chapter falls in one or more of the main software- 
engineering components in Figure 1.2.

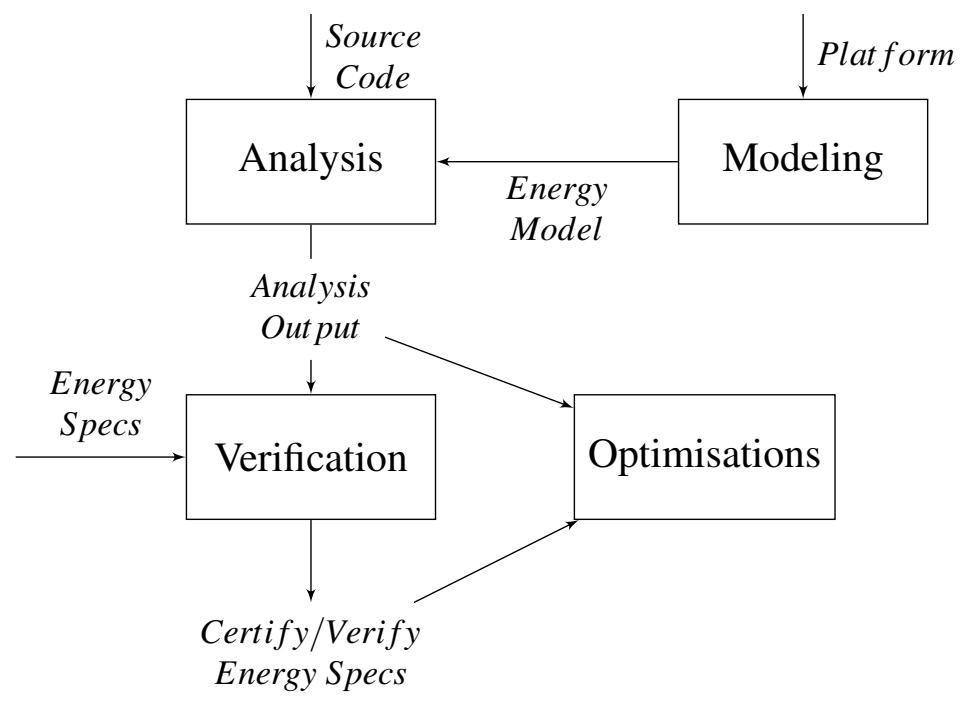

Figure 1.2: Energy-aware software engineering main components.

The multi-language energy consumption analysis and modeling framework is proposed in Chapter 2, by instantiating an existing static resource analysis framework. An average energy model is used that is fed to the framework via assertions. In Chapter 3 , the thesis describes a novel energy modeling technique that approximates both upper and lower bounds on the energy of branchless blocks of instructions. Then, such block-level model is used by the static analysis described in Chapter 2 to infer tight energy bounds that are practical for energy verification and optimization applications. Chapter 4 presents a novel resource analysis whose aim, compared to traditional static resource analysis, is the static profiling of accumulated cost, i.e., to discover, for selected parts of the program, an estimate or bound of the resource usage accumulated in each of those parts. In Chapter 5, energy-efficient scheduling and task allocation algorithms based on Evolutionary Algorithms (EAs) have been proposed that use the static analysis of tasks to obtain significant energy savings as well as to speed up the scheduling. A variation of such EA algorithm is also used to trade off accuracy versus energy for applications that permit certain levels of variability in the accuracy of their computations. Finally, some conclusions are drawn, and directions for future work are outlined in Chapter 6. 


\section{Multi-language Energy Consumption Analysis and Modeling Framework}

\subsection{Introduction}

In this chapter we propose a static analysis approach that infers energy consumption information at the ISA and LLVM IR levels, and reflects it up to the source code level. Such information is provided in the form of functions on input data sizes, and is expressed by means of assertions that are inserted in the program representation at each of these levels. The user (i.e., the "energy-efficient software developer") can customize the system by selecting the level at which the analysis will be performed (ISA or LLVM IR) and the level at which energy information will be output (ISA, LLVM IR or source code). As we will show later, the selection of analysis level has an impact on the analysis accuracy and on the class of programs that can be analyzed.

One of the main goals of this chapter is to study the feasibility and practicability of the proposed analysis approach and perform an initial experimental assessment to shed light on the trade-offs implied by performing the analysis at the ISA or LLVM levels. In our experiments we focus on the energy analysis of programs written in XC [95] running on the XMOS XS1-L architecture. However, the concepts presented here are neither language nor architecture dependent and thus can be applied to the analysis of other programming languages (and associated lower level program representations) and architectures as well. XC is a high-level C-based programming language that includes extensions for concurrency, communication, input/output operations, and realtime behavior. In order to potentially support different programming languages and different program representations at different levels of compilation (e.g., LLVM IR and ISA) in the same analysis framework we differentiate between the input language (which can be XC source, LLVM IR, or ISA) and the intermediate semantic program representation that the resource analysis operates on. The latter is a series of connected code blocks, represented by Horn Clauses, that we will refer to as "HC IR" from now on. We then propose a transformation from each input language into the HC IR and passing it to a resource analyzer. The HC IR representation as well as a transforma- 
tion from both ISA and LLVM IR into HC IR will be explained in Sections 2.2, 2.5.1 and 2.6.1 respectively. In our implementation we use an extension of the CiaoPP [42] resource analyzer. This analyzer always deals with the HC IR in the same way, independent of its origin, inferring energy consumption functions for all procedures in the HC IR program. The main reason for choosing Horn Clauses as the intermediate representation is that it offers a good number of features that make it very convenient for the analysis [67]. For instance, it supports naturally Static Single Assignment (SSA) and recursive forms, as will be explained later. In fact, there is a current trend favoring the use of Horn Clause programs as intermediate representations in analysis and verification tools $[34,45,22,12]$.

Although our experiments are based on single-threaded XC programs (which do not use pointers, since XC does not support them), our claim about the generality and feasibility of our proposed approach for static resource analysis is supported by existing tools based on the Horn Clause representation that can successfully deal with $\mathrm{C}$ source programs that exhibit interesting features such as the use of pointers, arrays, shared-memory, or concurrency in order to analyze and verify a wide range of properties [34, 45, 37]. For example [37] is a tool for the verification of safety properties of $\mathrm{C}$ programs which can reason about scalars and pointer addresses, as well as memory contents. It represents the bytecode corresponding to a $\mathrm{C}$ program by using (constraint) Horn clauses.

We also leverage an existing general framework for resource usage verification and specialize it for verifying energy consumption specifications. Such specifications can include both lower and upper bounds on energy usage, and they can express intervals within which energy usage is to be certified to be within such bounds. The bounds of the intervals can be given in general as functions on input data sizes. The verification system can prove whether such energy usage specifications are met or not by comparing the specifications with the approximated energy consumption information inferred by the static analysis.

Both static analysis and energy models can potentially relate to any language level (such as XC source, LLVM IR, or ISA). Performing the analysis at a given level means that the representation of the program at that level is transformed into the HC IR, and the analyzer "mimics" the semantics of instructions at that level. The energy model at a given level provides basic information on the energy cost of instructions at that level. The analysis results at a given level can be mapped upwards to a higher level, e.g. from ISA or LLVM IR to XC. Furthermore, it is possible to perform analysis at a given level with an energy model for a lower level. In this case the energy model must be reflected up to the analysis level.

\subsubsection{Hypothesis: Analysis and Modeling Trade-off}

Our hypothesis is that the choice of level will affect the accuracy of the energy models and the precision of the analysis in opposite ways: energy models at lower levels (e.g. at the ISA level) will be more precise than at higher levels (e.g. XC source code), since 
the closer to the hardware, the easier it is to determine the effect of the execution on the hardware. However, at lower levels more program structure and data type/shape information is lost due to lower-level representations, and we expect a corresponding loss of analysis accuracy. We could devise mechanisms to represent such higher-level information and pass it down to the lower-level ISA, or to recover it by analysing the ISA. However, our goal is to compare the analysis at the LLVM IR and ISA levels without introducing such mechanisms, which might be complex or not effective in some cases (e.g., in abstracting memory operations or recovering type information).

This hypothesis about the analysis/modeling level trade-off (and potential choices) was illustrated in Figure 1.1. The possible choices are classified into two groups: those that analyze and model at the same level, and those that operate at different levels. For the latter, the problem is finding good mappings between software segments from the level at which the model is defined up to the level at which the analysis is performed, in a way that does not lose accuracy in the energy information.

In this chapter we concentrate on two of these choices and their comparison, to see if our hypothesis holds. In particular, the first approach (choice 1) is represented by analysing the generated ISA-level code using models defined at the ISA level that express the energy consumed by the execution of individual ISA instructions. We explored this approach first in [62] that is detailed in Section 2.5. It used the precise ISA-level energy models presented in [51], which when used in the static analysis for a number of small numerical programs resulted in the inference of functions that provide reasonably accurate energy consumption estimations for any input data size $(3.9 \%$ average error vs. hardware measurements). However, when dealing with programs involving structured types such as arrays, it also pointed out that, due to the loss of information related to program structure and types of arguments at the ISA level (since it is compiled away and no longer relates cleanly to source code), the power of the analysis was limited. In Section 2.6, we also explore an alternative approach: the analysis of the generated LLVM IR (which retains much more of such information, enabling more direct analysis as well as mapping of the analysis information back to source level) together with techniques that map segments of ISA instructions to LLVM IR blocks [31] (choice 2). This mapping is used to propagate the energy model information defined at the ISA level up to the level at which the analysis is performed, the LLVM IR level. In order to complete the LLVM IR-level analysis, we have also developed and implemented a transformation from LLVM IR into HC IR and used the CiaoPP resource analyzer. This results in a parametric analysis that similarly to ISA analysis (Section 2.5) infers energy consumption functions, but operating on the LLVM IR level rather than the ISA level.

We have performed an experimental comparison of the two choices for generating energy consumption functions. Our results support our intuitions about the trade-offs involved. They also provide evidence that the LLVM IR-level analysis (choice 2) offers a good compromise within the level hierarchy, since it broadens the class of programs that can be analyzed without the need for developing complex techniques for recovering type information and abstracting memory operations, and without significant loss 
of accuracy.

In summary, the original contributions of this chapter are:

1. Translations from ISA and LLVM IR to HC IR (Section 2.5.1 and 2.6.1).

2. The integration of all components into an experimental tool architecture, enabling the static inference of energy consumption information in the form of functions on input data sizes and the experimentation with the trade-offs described above (Section 2.6). The components are: LLVM IR and ISA translations, ISA-level energy model and mapping technique (Section 2.6.2 and [51, $31]$ ), and analysis tools (Section 2.6.3 and [77, 87]).

3. The experimental results and evidence of trade-off of precision versus analyzability (Section 2.7).

4. A sketch of how the static analysis system can be integrated in a source-level Integrated Development Environment (IDE) (Section 2.5 and 2.6).

5. Leverage an existing general framework for resource usage verification and specialize it for verifying energy consumption specifications (Section 2.8).

Finally, Section 2.9 discusses some related work and Section 2.10 summarises our conclusions and comments on ongoing and future work.

\subsection{Analysis and Modeling Framework Architecture}

Figure 2.1 shows a high-level view of the general analysis framework that we propose, illustrating the ideas described above. The process starts with a source program that may contain assertions (expressed in the front end part of the common assertion language, and used to provide useful hints and information to the analyser), from which the Transformation tool (red box) generates its associated HC IR (green box). We assume that the Transformation box includes the compilation process. Thus, the idea is that the transformation box takes the source code as input and produces the program to be analysed. The transformation process is also in charge of translating the (front end) assertions (or annotations) present in the source code into assertions written in the Ciao assertion language. Moreover, the transformation process takes an energy model as input, and translates it into assertions expressed in the Ciao assertion language. The role of the energy models is to express the effect, in terms of energy consumption or other resources that depend on the underlying hardware, of the execution of a software segment (e.g., an assembly instruction) on the hardware. Such information is required by the analyser, which propagates it during the static analysis of a program (expressed in the HC IR) in order to infer information (the analysis results) for higher-level entities such as functions or procedures in the program. This is also illustrated in Figure 2.1, where the Analyser (blue box) takes the HC IR, together with the Ciao assertions, expressing the energy models and possibly useful (trusted) information, and processes 


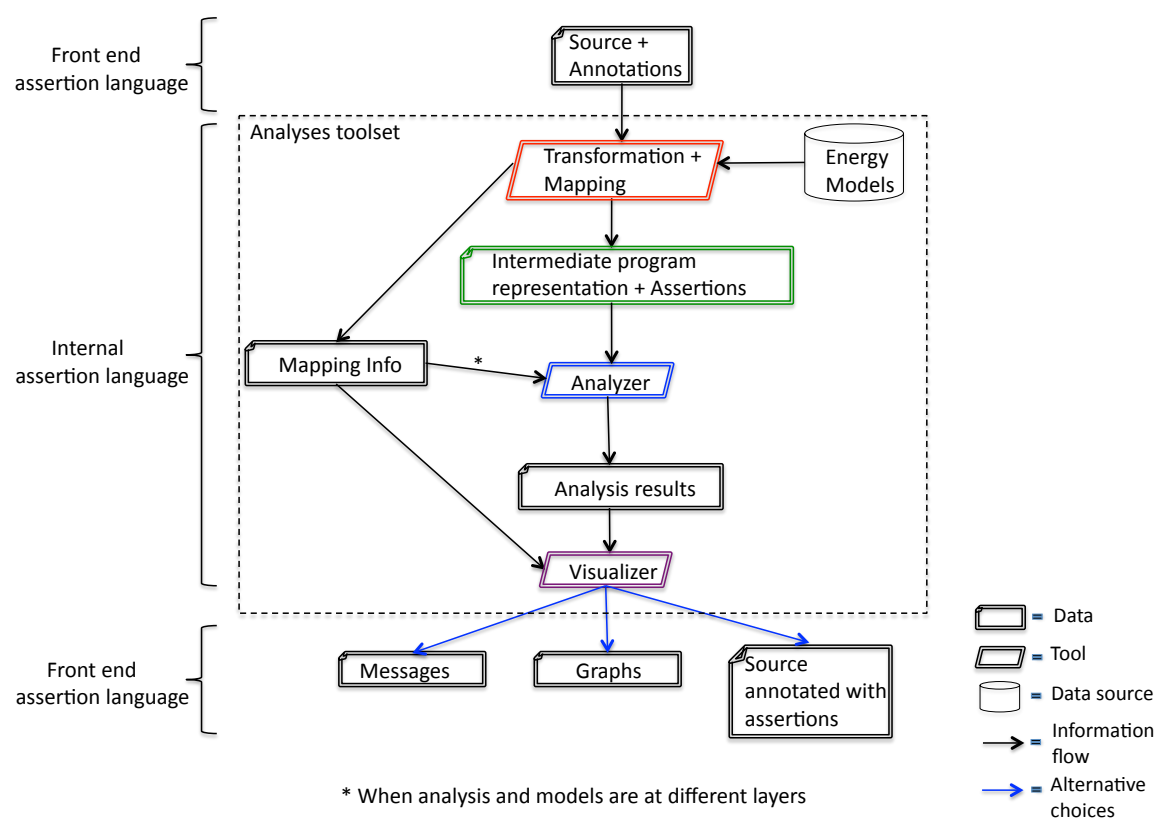

Figure 2.1: High-level view of the general analysis framework architecture.

them, producing the analysis results, expressed also in the Ciao assertion language. The analysis results include energy consumption (or, in general, resource usage) information expressed as functions on data sizes for the whole program and parts of it, such as procedures and functions, as mentioned before. Such results, are then processed by a Visualiser (pink box) which is in charge of showing the information to the users (system developers) in an appropriate format, in order to help them to better understand the effect of their designs on the energy consumption early on during the software development process, and make more informed design decisions (e.g., using the appropriate data structures), even when there are parts not developed yet.

Figures 1.1 and 2.2 illustrate the idea that the analysis can be performed at the three layers mentioned before. Figure 2.2 provides a more detailed view of the analysis/modeling layers within the analysis framework shown in Figure 2.1. For simplicity, Figure 2.2 shows the models defined at the same layer the analysis is performed. At any of these layers, a transformation into the HC IR is carried out, and the HC IR is then passed to the analyser. These alternative choices for the "Transformation box" are represented in the figure by using blue arrows. Note that in order to map the analysis information inferred at one layer to an upper layer (typically to the source code layer) the (program dependent) mapping information, produced also by the transformation process, is used. However, this mapping process does not perform any analysis in principle. Such mapping information is different from the (program dependent) mapping information that can be used by the analyser to propagate the energy model 
information defined at one layer up to the (different) layer at which the analysis is performed.

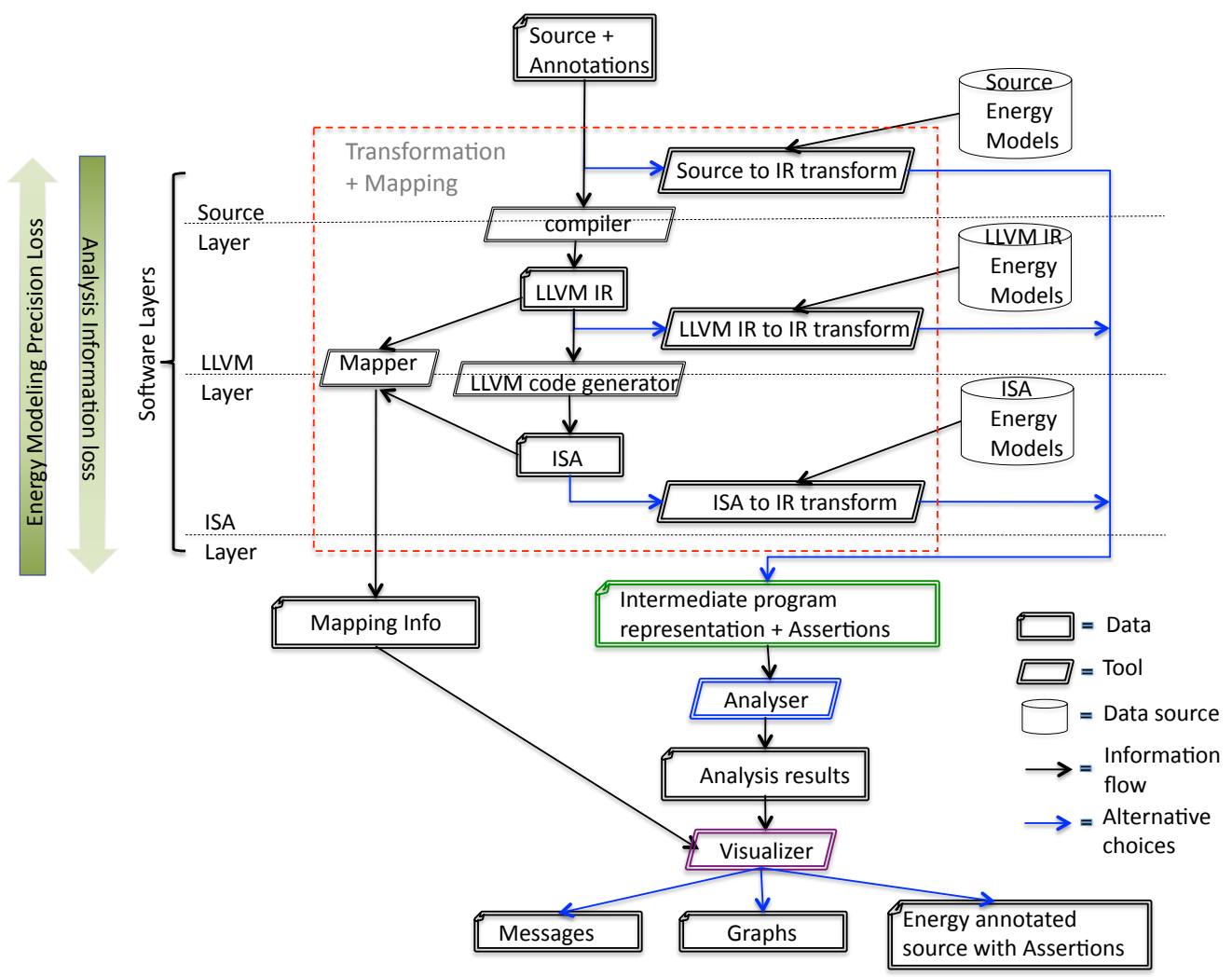

Figure 2.2: Detailed view of the analysis/modeling layers within the analysis framework.

Intermediate Semantic Program Representation for Analysis (HC IR). As already said, the key to handle different languages in the same framework is to use an Intermediate Semantic Program Representation for Analysis based on Horn clauses, $H C I R$, and perform a transformation from each source code into the HCIR.

Definition 1. A Horn clause (HC) is a first-order predicate logic formula of the form $\forall\left(S_{1} \wedge \ldots \wedge S_{n} \rightarrow S_{0}\right)$ where all variables in the clause are universally quantified over the whole formula, and $S_{0}, S_{1}, \ldots, S_{n}$ are atomic formulas, also called literals. It is usually written $S_{0}:-S_{1}, \ldots, S_{n}$.

The HC IR representation consists of a sequence of blocks. Each block is represented as a Horn clause:

$$
<\text { block_id }>(<\text { params }>):-S_{1}, \ldots, S_{n} \text {. }
$$


which has an entry point, that we call the head of the block (to the left of the :symbol), including a number of parameters $<$ params $>$, and a sequence of steps (the body, to the right of the :- symbol), each of which is either, (the representation of) an ISA or LLVM IR instruction (depending on the layer at which the program is analysed), or a call to another (or the same) block. Be it LLVM IR, ISA, or any other program representation, the analyser deals with the HC IR always in the same way, independently of its origin.

\subsection{Static Resource Analysis}

Resource usage analysis infers the aggregation of some numerical properties (named resources), like memory usage, time spent in computation, energy consumption or bytes sent over a socket, throughout the execution of a piece of code. This information is returned in the form of functions of the sizes of some input arguments.

The starting point of this approach is the methodology outlined by [26, 25, 24], characterized by the setting up of recurrence equations. This methodology basically performs, previous to the resource usage analysis, a size and cardinality analysis for inferring size relations and bounds on the number of solutions computed by a predicate $p$, respectively. This information is ultimately used to obtain the resource usage bound functions.

Definition 2. A resource usage bound function for $p$ is a monotonic arithmetic function, $\Psi_{p}: S \mapsto \mathcal{R}_{\infty}$, for a given subset $S \subseteq \mathcal{R}^{k}$, where $\mathcal{R}$ is the set of real numbers, $k$ is the number of input arguments to predicate $p$, and $\mathcal{R}_{\infty}$ is the set of real numbers augmented with the special symbols $\infty$ and $-\infty$. We use such functions to express lower and upper bounds on the resource usage of predicate $p$ depending on its input data sizes.

\subsubsection{CiaoPP Resource Analysis}

In order to perform the global energy consumption analysis, our approach leverages the CiaoPP tool [42], the preprocessor of the Ciao programming environment [40]. CiaoPP includes a global static analyzer which is parametric with respect to resources and type of approximation (lower and upper bounds) [77, 87]. The framework can be instantiated to infer bounds on a very general notion of resources, which we adapt in our case to the inference of energy consumption. The basic approach is as follows. Given a predicate call $p$, let $\Phi(p, r, \bar{n})$ denote the exact units of resource $r$ consumed or produced during the computation of $p$ for a tuple of argument sizes $\bar{n}$. Note that, in general, the computation of $\Phi(p, r, \bar{n})$ will be undecidable or very complex. Therefore, an expression $\operatorname{Cost}(p, a p, r, \bar{n})$ is determined at compile-time that approximates $\Phi(p, r, \bar{n})$ with approximation ap (i.e., upper-bound or lower-bound). For assuring the correctness the analysis always generate resource usage bounds functions such as $\operatorname{Cost}(p, a p, r, \bar{n})$ that hold the following conditions: 
- If the analysis computes an upper-bound approximation, i.e., $a p=\mathrm{ub}$, then:

$$
\Phi(p, r, \bar{n}) \leq \operatorname{Cost}(p, \mathrm{ub}, r, \bar{n})
$$

- Conversely, if the analysis computes a lower-bound $a p=1 \mathrm{~b}$, then:

$$
\operatorname{Cost}(p, 1 \mathrm{~b}, r, \bar{n}) \leq \Phi(p, r, \bar{n})
$$

Note that the analysis can always generate trivial upper and lower bounds, $\infty$ and $-\infty$, in those cases where it cannot infer resource equations or find a closed form. Of course, the analysis should infer bounds as precise as possible.

Certain program information is first automatically inferred by other abstract interpretationbased analyzers included in CiaoPP and then provided as input to the size and resource analysis:

1. Inference of modes, i.e., determine which arguments are input or output.

2. Inference of types for each predicate argument.

3. Inference of size metrics for predicate arguments based on the type information.

4. Inference of non-failure information, i.e., determine which predicates should fail.

The resource analysis in CiaoPP works on the intermediate block-based representation language, which we have called HC IR in this chapter. Each block is represented as a Horn Clause, so that, in essence, the HC IR is a pure Horn clause subset (pure logic programming subset) of the Ciao programming language.

In CiaoPP, a resource is a user-defined counter representing a (numerical) nonfunctional global property, such as execution time, execution steps, number of bits sent or received by an application over a socket, number of calls to a predicate, number of accesses to a database, etc. The instantiation of the framework for any particular resource is done by means of an assertion language that allows the user to define resources and other parameters of the analysis by means of assertions. Such assertions are used to assign basic resource usage functions to elementary operations and certain program constructs of the base language, thus expressing how the execution of such operations and constructs affects the usage of a particular resource. The resource consumption provided can be a constant or a function of some input data values or sizes. The same mechanism is used as well to provide resource consumption information for procedures from libraries or external code when code is not available or to increase the precision of the analysis. 


\subsection{Energy Characterization and Modeling}

The assertion-based model uses power consumption data collected during hardware measurement. We have used an ISA-level model that provides software energy consumption estimates based on Instruction Set Simulation (ISS) statistics. The hardware, the measurement process, as well as the construction of the ISS-driven model, are detailed in Kerrison et al. [51], with the key components relevant to this chapter explained in the rest of this section as well as in the published collaboration [62].

The practicality and accuracy of this approach to energy consumption analysis relies on a good characterisation of energy consumption and generating good energy consumption models. A trade-off needs to be found between the simplicity of the models, which improves the efficiency of the analysis, and the accuracy of the models, which improves the accuracy of the global analysis. Although we analyse singlethreaded code, the energy profiling must consider the hardware multi-threading of the architecture, which has an energy impact even when only a single thread is executed.

Further, the nature of the architecture requires specific approaches in order to gather energy profiling data, but these same characteristics preclude certain energy effects from static analysis. For example, the effects of interleaving instructions or re-use of operands from the previous instruction become less relevant in a hardware multithreaded pipeline, and impossible to determine statically. Although manifested in a specific way in this particular processor architecture, such traits also exist in other processors, such as super-scalar designs. In this chapter we describe an initial proposal that offers a good compromise between the above issues, and also eliminates factors that are determined to be insignificant.

\subsubsection{Energy Profiling Framework and Strategy}

An energy profiling framework, xmprofile, is used to generate sequences of instructions under various constraints in order to profile the energy characteristics of the hardware. This data is essential for the accurate application of models at any analysis level. The hardware used is shown in Figure 2.3. A master processor issues test programs to and measures the power used by a slave processor, the Device Under Test (DUT).

Currently, a subset of the ISA, including arithmetic operations, logic operations, and condition tests, has been characterised. Other instructions are at the moment approximated using a single average value, based on typical observed behaviour.

\subsubsection{ISA-level Model}

An ISA-level model, xmmodel, gives an energy estimate for a program based on ISS output. Data from the measurement framework feeds this model.

Kerrison et al.'s model is based on that devised by Tiwari [91]. Tiwari's approach is shown in Equation (2.3). The energy of an ISA program, $E_{p}$, is characterised as the sum of base energy cost, $B_{i}$, for all ISA instructions, $i$, multiplied by the number of 
executions of each instruction, $N_{i}$. An inter-instruction overhead energy, $O_{i, j}$, is then accounted for by enumerating for all instruction combinations $i, j$ and their frequency, $N_{i, j}$. Finally, additional contributions to program energy can be accounted for by $k$ external effects, $E_{k}$, which may include externally modelled behaviours such as cache memory.

$$
E_{p}=\sum_{i \in \mathrm{ISA}}\left(B_{i} \times N_{i}\right)+\sum_{i, j \in \mathrm{ISA}}\left(O_{i, j} \times N_{i, j}\right)+\sum_{k \in \mathrm{ext}} E_{k}
$$

The XS1 architecture is hardware multi-threaded. This necessitates a fundamental revision of the model equation. In addition, for performance reasons, the ISS collects instruction statistics rather than a full trace. This reduces the execution time by an order of magnitude, such that it is approximately 100 times slower than the hardware when simulation is run on a modern computer.

Equation (2.4) describes the energy of a program, $E_{p}$, using a similar method to Equation (2.3), but with several key differences. Time is an explicit component, multiplied by power terms in order to calculate energy. This separation enables future exploration of idle periods, external event timing, and variable operating frequencies. Inter-instruction overhead is represented as a single component, rather than considering it for all possible pairs of instructions, on account of a statistics-based approach rather than cycle-by-cycle instruction tracing. Finally, the level of concurrency must be accounted for, something that was not necessary for the architecture targeted by Equation (2.3). The concurrency level is the number of threads that are active at a given time. In the case of the XS1-L, the concurrency level represents how full the pipeline is and therefore how much activity is generated within it as each stage switches between instructions from the active threads.

$$
E_{\mathrm{p}}=P_{\text {base }} N_{\text {idle }} T_{\text {clk }}+\sum_{t=1}^{N_{t}} \sum_{i \in \mathrm{ISA}}\left(\left(M_{t} P_{i} O+P_{\text {base }}\right) N_{i, t} T_{\text {clk }}\right)
$$

The base power, $P_{\text {base }}$, is present in both active and idle periods. The number of idle periods, $N_{\text {idle }}$, is counted and multiplied by the clock period, $T_{c l k}$, to account for the energy consumed when no threads are active. For each number of concurrent threads,

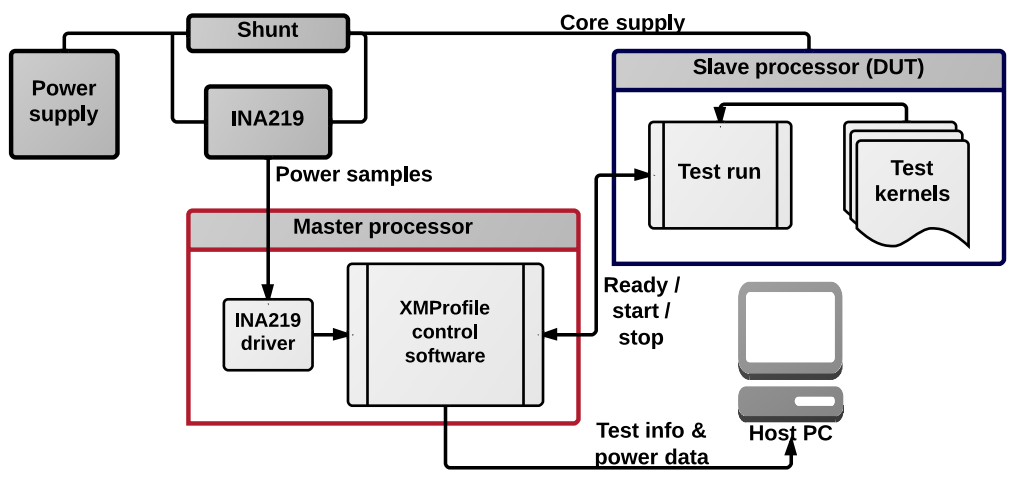

Figure 2.3: Overview of test harness hardware and software structure, with a slave processor executing test kernels and a master processor collecting power samples. 
$t$, (based on the proportion of time each thread is active), and for each instruction, $i$, in the ISA, the instruction power, $P_{i}$, is multiplied by a constant inter-instruction power overhead, $O$, and a concurrency cost for the level of concurrency at which the processor is operating, $M_{t}$. These are all multiplied by the number of times this instruction occurs at this concurrency level, $N_{i, t}$, and the clock period. Combined with the idle energy, this gives a total energy estimate for the program run.

In the case where a single thread is running, with no idle periods, then the above can be simplified to Equation (2.5). The result is very similar to the single-threaded Tiwari equation, but with only a single, generic inter-instruction power overhead component, $O$, and with no external " $k$ " components as the memory of the XS1-L is single-cycle with no cache, with no other effects that need to be considered at this point. There is only ever one active thread, so we use the concurrency cost for one thread, $M_{1}$. Again, in Equation (2.5), time is an explicit component. The overhead, $O$, is a constant because the inter-instruction effect cannot be known statically in the XS1 architecture, and during profiling the variation in inter-instruction effect was shown to be an order of magnitude less than the instruction cost and would average out over program runs.

$$
E_{\mathrm{p}}=\sum_{i \in \mathrm{ISA}}\left(\left(M_{1} P_{i} O+P_{\text {base }}\right) \times\left(N_{i} T_{\mathrm{clk}}\right)\right)
$$

Kerrison et al.'s ISS-based model, using the same energy data as the static analysis, will be used as an additional comparison point between actual hardware energy measurements and the static analysis results.

\subsection{Analysis at the ISA level}

We first have explored the choice of both analysing and modeling at the ISA level and have realized the general analysis framework in Figure 2.1. This is illustrated in Figure 2.4, as a realization of Figure 2.1.

The system takes as input an XC source program that can (optionally) contain assertions (used to provide useful hints and information to the analyzer), from which a Transformation and Mapping process (dotted red box) generates first its associated ISA using the xcc compiler. Then, a transformation from ISA into HC IR is performed (explained in Section 2.5.1) obtaining the intermediate representation (green box) that is supplied to the CiaoPP analyzer. This representation includes assertions that express the energy consumed by the ISA instructions. The CiaoPP analyzer (blue box, described in Section 2.6.3) takes the HC IR, together with the assertions which express the energy consumed by ISA instructions, and possibly some additional (trusted) information, and processes them, producing the analysis results, which are expressed also using assertions. Based on the procedural interpretation of these HC IR programs and the resource-related information contained in the assertions, the resource analysis can infer static bounds on the energy consumption of the HC IR programs that are applicable to the original ISA and, hence, to their corresponding XC programs. The analysis results include energy consumption information expressed as functions on data sizes 


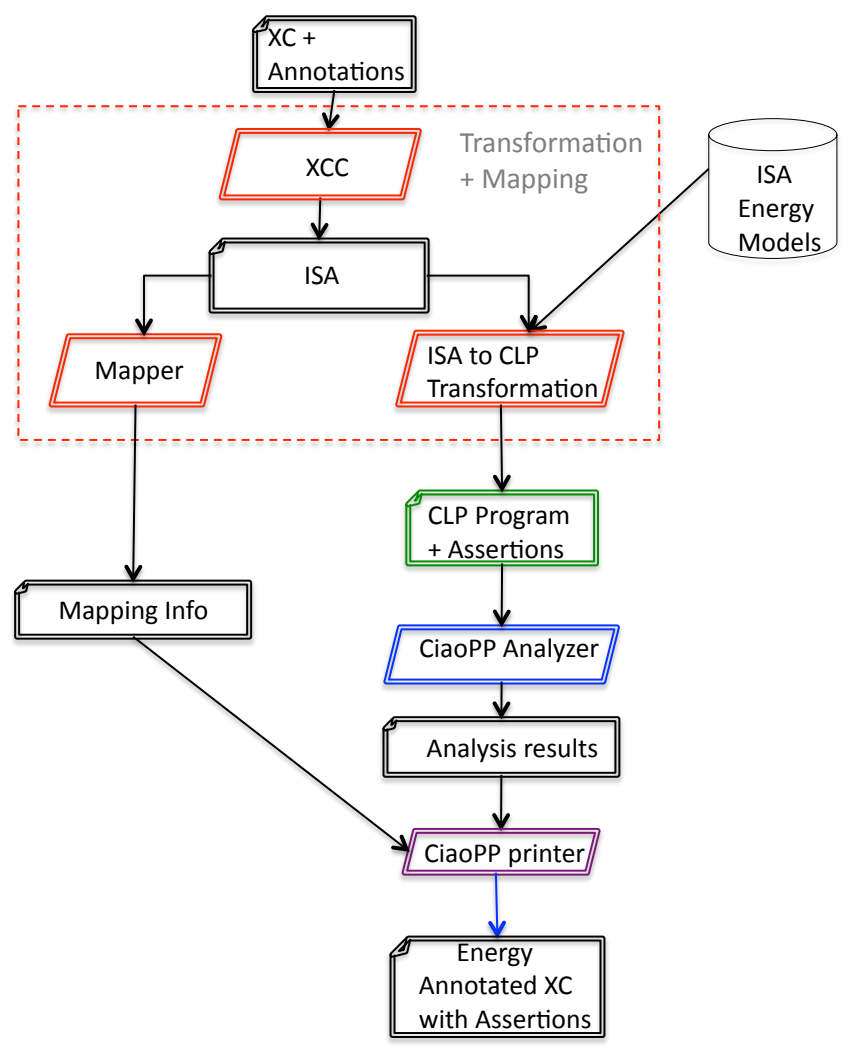

Figure 2.4: A realization of the general analysis framework using models and performing the analysis at the ISA layer.

for the whole program and for all the procedures and functions in it. Such results are then processed by the CiaoPP printer (purple box) which presents the information to the program developer in a user-friendly format.

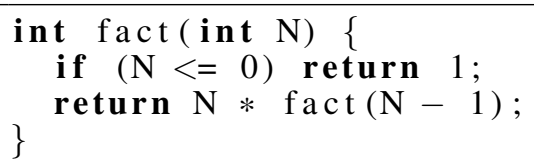

Figure 2.5: An XC source (factorial) function.

Consider the fact function in Figure 2.5 as the XC program for the energy consumption analysis using the framework. The ISA program corresponding to it is generated using the XC compiler tool XCC (left hand side of Figure 2.6). The resulting ISA program is passed to a translator which generates the associated Horn clauses (right hand side of Figure 2.6). Such program, together with the information contained in the energy models at the ISA level (represented using the mentioned assertion language), is passed to the resource analysis which outputs the energy consumption for all procedures in the HC IR program. In our example, the resource analysis infers an estimation 
control flow graph is constructed by the analyser, where each node represents a block. Edges between the blocks are derived from calls/jumps between blocks. This process involves iterating through the CFG of the ISA program and marking block boundaries, which are instructions that either begin or end a basic block.

\subsubsection{Inferring Block Input/Output Parameters.}

In order to treat each block as a Horn clause, the block's input and output arguments need to be inferred. For the entry block, the input and output arguments are derived from the original function's signature. We define the functions params in and params $_{\text {out }}$, which infer input and output parameters of a block respectively. These perform a backwards analysis of the program, and are recomputed until a least fixpoint is reached on these functions.

$$
\begin{aligned}
\operatorname{params}_{\text {out }}(b) & =\operatorname{kill}(b) \cup \bigcup_{b^{\prime} \in \text { next }(b)} \operatorname{params}_{\text {out }}\left(b^{\prime}\right) \\
\operatorname{params}_{\text {in }}(b) & =\operatorname{gen}(b) \cup \bigcup_{b^{\prime} \in \operatorname{next}(b)} \operatorname{params}_{\text {in }}\left(b^{\prime}\right)
\end{aligned}
$$

where next $(b)$ denotes the set of immediate target blocks that can be reached from $b$ with a call or jump, while $g e n(k)$ and $k i l l(k)$ are the read and written variables in a block respectively, which we define as:

$$
\operatorname{kill}(b)=\bigcup_{k=1}^{n} \operatorname{def}(k), \quad \operatorname{gen}(b)=\bigcup_{k=1}^{n}\{v \mid v \in \operatorname{ref}(k) \wedge \forall(j<k) \cdot v \notin \operatorname{def}(j)\}
$$

and $\operatorname{def}(k)$ and $r e f(k)$ denote the variables written or referred to at a node in the block respectively.

Our approach here is closely related to that of the live variable analysis (LVA) [78] used in compilers, and in dead code elimination in particular. A variable is live at a program point if it may get referenced later in the program (which is decided by considering the whole CFG of the program). In LVA, for each program point, a set of live variables is computed using functions similar to our kill and gen functions with data flow equations. In our approach however, instead of computing liveness information for each program point, we compute a least fixpoint of our params out $_{\text {and }}$ and params $_{\text {in }}$ functions over the program's block control flow graph. This is an efficient solution that safely over-approximates the set of input/output arguments to each block, so that the extra arguments inferred for block heads due to such over-approximation do not affect the energy consumption estimations, since they are not used in the analysis of procedures corresponding to the original $\mathrm{XC}$ code.

\subsubsection{Resolving Branching to Multiple Blocks.}

In the XS1 instruction set, conditional branch instructions (e.g., bt, bf) jump to one of the two target blocks based on the value of the branching variable. For example, in Figure 2.6, at line 7 the bf instruction (branch if fail) will jump to address 008 if 
$r 0=0$, otherwise to address 007 . In the HC IR this branch needs to be a call to one of the two blocks.

We use a similar approach to the one described in [67] to resolve branches to multiple blocks. The multiple target blocks of a jump instruction are assigned the same head, which essentially are clauses of the same HC IR predicate. This is achieved by merging the heads of the target clauses so that each clause has the same head. The algorithm is trivial, since we have already inferred the input/output parameters to each block's head. The input/output parameters to the new head of the clauses are the union of the input/output parameters of all the clauses along with the branching variable. This enables preservation of the branching semantics of the original ISA program in the HC IR form.

For example in Figure 2.6, the bf instruction at line 7 of the ISA program is changed to a dummy literal at line $7 \mathrm{a}$ in the HC IR, plus a predicate call to fact_aux on line $7 \mathrm{~b}$. The predicate fact_aux has two clauses, each representing one of the target blocks of the bf instruction. The dummy literal for the bf instruction is created so that the resource usage analysis can take it into account when inferring energy usage functions.

\subsubsection{Static Single Assignment form (SSA).}

The last step is to convert the block representation into static single assignment (SSA) form, where each variable is assigned exactly once and multiple assignments to the same variable create new versions of that variable.

In compilers, the SSA form is generated at the function level (e.g., at LLVM [55] level) where a function might consist of multiple basic blocks. However, we follow the approach of generating the SSA form at the block level, and therefore we do not need to generate $\phi$ nodes. A $\phi$ node is an instruction used to select a version of the variable depending on the predecessor of the current block. Since each block is already annotated with input/output arguments, any predecessor block will pass the appropriate values as input parameters when making a call to the target block.

In Figure 2.6, the HC IR (right hand side) is already in SSA form, where each variable is defined exactly once and stack references are transformed to local variables. Each instruction is transformed into a HC IR literal with input/output variables.

Analysis on low level (ISA) representations, in general, suffers from the problem of extracting a precise control flow graph in the presence of indirect jumps and calls. The current implementation of our transformation is restricted to direct jumps and calls. We plan to integrate other techniques into the transformation tool to resolve such problems including recognizing code patterns used by compilers and performing static program analysis (see [101] and its references).

\subsubsection{Instantiating the CiaoPP Analysis Framework}

In this section we describe how to instantiate the CiaoPP analysis framework for the analysis of the HC IR programs resulting from the translation of ISA programs. we use 
a running example to illustrate the main concepts and steps of the analysis framework. In particular, and for simplicity, assume that we are interested in estimating upper bounds on the energy consumed by the HC IR program in Figure 2.6 (right hand side) generated from its XC

\subsubsection{Defining Resources.}

We start by defining the identifier ("counter") associated to the energy consumption resource, through a declaration:

:- resource energy.

\subsubsection{Expressing the Energy Model.}

In CiaoPP, the resource usage of primitive operations can be provided using "trust" assertions (see [40] and its references for a description of the assertion language). For example, we can write assertions for each predicate that represents an ISA instruction; these constitute the energy models. The following assertions (for the add and sub instructions) are part of the simple energy model that we used in the static analysis, which assigns a constant energy consumption to these ISA instructions (values 1215439 and 1210759 respectively):

$:-$ trust pred add $(X, Y, Z)+$ resource(avg, energy, 1215439).

$:-$ trust pred sub $(X, Y, Z)+$ resource (avg, energy, 1210759).

Note that the first argument (avg) of the resource property (in the global computational properties field "+" of the assertions) expresses that the given energy consumption for the ISA instructions is an average value. This model is obtained using the measurement process described in Section 2.4, based on Equation (2.5), so that the energy cost for an ISA instruction $i$ is $c_{i}=\left(M_{1} P_{i} O+P_{\text {base }}\right) T_{\text {clk }}$, expressed in the third argument of the resource property in femto-Joules (fJ, $10^{-15}$ Joules).

Assertions are also used to express other information that is instrumental in the resource usage analysis. For example, the assertion:

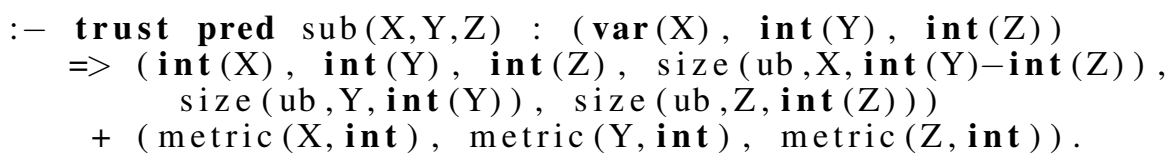

indicates that if the sub $(X, Y, Z)$ predicate (representing the "subtraction" ISA instruction) is called with $X$ and $Y$ bound to integer numbers and $Z$ an unbound variable (precondition field “:”), after the successful completion of the call (postcondition field " $=>$ "), $X$ is an integer number whose size is the size of $Y$ minus the size of $\mathrm{Z}$. It also expresses that the size metric used for the three arguments is "int", the actual value of the integer numbers. 


\subsubsection{Performing the Analysis}

Once the parameters of the general resource analysis framework have been defined, and assertions for primitives (representing the energy models) and library calls have been provided, the CiaoPP global static analysis can infer the resource usage of all the procedures/blocks in the program (as functions of input data sizes). A full description of how this is done can be found in [77].

\subsubsection{Calling Mode Information.}

The resource analysis needs information referred to each argument in each predicate in the block representation (HC IR) that expresses whether it acts as an input or an output argument (its "mode"). In our approach no mode analysis is performed in order to obtain such information. The modes of the main blocks are extracted from the $\mathrm{XC}$ source code that the HC IR is originated from. This is possible because mode information is statically known at the $\mathrm{XC}$ language level and is propagated to the $\mathrm{HC}$ IR using (trust) assertions. There are also new intermediate predicates generated by the transformation from ISA programs into HC IR (described in Section 2.5.1), originated from conditional branching, which cannot be directly related to the $\mathrm{XC}$ source code. However, for such predicates information from the transformation phase, where the input/output arguments are determined for each predicate, is used, so that no mode analysis needs to be performed by CiaoPP.

\subsubsection{Size Measure Analysis.}

CiaoPP uses type information to decide which metric to use to infer and express data sizes, from a set of predefined metrics (see [77] for details). As already said, our resource analysis is performed on a block-based representation (HC IR) of the ISA code generated by the XC compiler. Although $\mathrm{XC}$ is a typed language, most of the type information is lost in the ISA code generated by the compiler. There are a number of static and dynamic techniques developed by the reverse engineering community to reconstruct types/shape information from binaries (see [56] and its references). In our approach, we can recover and transfer types from the ISA code into some blocks (predicates) in the HC IR that are directly related to the ISA code, so that no type analysis is performed in those cases. However, we still need to perform some propagation of such types to any new intermediate blocks created by the transformation from ISA programs into Horn clauses. For example, our approach can determine that in the HC IR program in Figure 2.6 (right hand side) fact will be called with $R 0$ bound to an integer and $R 0 \_3$ a free variable, and will succeed with $R 0 \_3$ bound to an integer. Also, fact_aux will be called with the first two arguments bound to integers, and the rest free, and, upon success, all of them will be bound to integers. Given that information, the chosen metric for all the arguments will be int, i.e., the integer value of the argument. 


\subsubsection{Size Analysis.}

It determines the relative sizes of variable bindings at different program points. For each clause, size relations are propagated to express each output data size as a function of input data sizes. For recursive functions this is done symbolically, creating a set of recurrence relations that will be solved to get a closed form function.

For our running example, the recurrence relations set up for the size of the output argument $R 0 \_3$ of fact as a function of the size of the input argument $R 0$ (denoted $\left.\mathrm{fact}_{R 0 \_3}(R 0)\right)$ as well as the corresponding one for fact_aux are:

$$
\begin{aligned}
& \operatorname{fact}_{R 0 \_3}(R 0)=\text { fact_aux } R_{R 0 \_4}(0 \leq R 0, R 0) \\
& \text { fact_aux }{ }_{R 0 \_4}(B, R 0)=\left\{\begin{array}{lr}
R 0 * \operatorname{fact}_{R 0_{-} 3}(R 0-1) & \text { if } B \text { is true (i.e., } 0 \leq R 0) \\
1 & \text { if } B \text { is false (i.e., } 0>R 0)
\end{array}\right.
\end{aligned}
$$

These inferred recurrence relations/equations are then fed into a computer algebra system (e.g., CiaoPP's internal solver or an external solver such as Mathematica, used for the results presented in this chapter) that gives the following closed form function for it: $\operatorname{fact}_{R 0}{ }_{3}(R 0)=R 0$ !

\subsubsection{Resource Usage Analysis.}

It uses the size information inferred by the size analysis to set up recurrence equations representing the resource usage of predicates (blocks), and computes bounds to their solutions. Remember that $c_{i}$ represents the energy cost of each instruction, taken from the energy model. Let $b_{e}$ denote the energy consumption function for a predicate (block) b. Then, the inferred equations for fact are:

$$
\begin{gathered}
\operatorname{fact}_{e}(R 0)=\text { fact_aux }_{e}(0 \leq R 0, R 0)+c_{\text {entsp }}+c_{s t w}+c_{l d w}+c_{l d c}+c_{l s s}+c_{b f} \\
f_{a c t} \operatorname{aux}_{e}(B, R 0)=\left\{\begin{array}{cr}
\operatorname{fact}_{e}(R 0-1)+c_{b u}+2 c_{l d w}+c_{s u b}+ & \text { if } B \text { is true } \\
+c_{b l}+c_{m u l}+c_{r e t s p} & \text { if } B \text { is false } \\
c_{\text {mkmsk }}+c_{\text {retsp }}
\end{array}\right.
\end{gathered}
$$

If we assume (for simplicity of exposition) that each instruction has unitary cost, i.e., $c_{i}=1$ for all $i$, we obtain (using the mentioned computer algebra system) the energy consumed by fact as a function of its input data size $(R 0):$ fact $_{e}(R 0)=13 R 0+$ 8.

Note that our approach based on setting up recurrence equations and solving them using a computer algebra system allows inferring different types of (resource usage) functions, such as polynomial, factorial, exponential, logarithmic, and summatory.

Note also that using average energy values in the model implies that the energy function for the whole program inferred by the upper-bound resource analysis is an approximation of the actual upper bound (possibly below it). Thus, theoretically, to ensure that the analysis infers an upper bound, we need to use upper bounds as well in the energy models. This is not a trivial task as the worst case energy consumption depends on the data processed, is likely to be different for different instructions, and 
Table 2.1: Description of benchmark functions used in experiments and their corresponding energy functions.

\begin{tabular}{|c|l|c|}
\hline Function name & Description & Energy function \\
\hline fact (N) & Calculates $N !$ & $26.0 N+19.4$ \\
\hline fibonacci (N) & Nth Fibonacci no. & $30.1+35.6 \phi^{N}+11.0(1-\phi)^{N}$ \\
\hline sqr (N) & Computes $N^{2}$ & $103.0 N^{2}+205.8 N+188.32$ \\
\hline poweroftwo (N) & Calculates $2^{N}$ & $62.4 \cdot 2^{N}-312.3$ \\
\hline power (base, exp) & Calculates base exp $^{2}$ & $6.3\left(\log _{2} \exp +1\right)+6.5$ \\
\hline
\end{tabular}

unlikely to occur frequently in subsequent instructions. We discuss this further in Chapter 3 and propose a novel approach of modeling blocks of instructions as a unit of modeling instead of the instruction.

\subsubsection{Benchmarks, Results and Evaluation}

The aim of the experimental evaluation is to perform a first comparison of actual hardware energy measurements, in terms of accuracy, with the values obtained from both the low-level Instruction Set Simulation (ISS) model and the Static Resource Analysis (SRA) implemented within the CiaoPP framework, to obtain an early estimation of the feasibility of the approach. To this end, we describe a selection of currently analysable benchmarks, the method by which data was collected, and an evaluation of the analysis framework accuracy vs. the low-level ISS model and hardware measurements.

\subsubsection{Benchmarks.}

For this type of evaluation we use as benchmarks mainly small mathematical functions. The structure of these programs is either iterative or recursive, with their cost depending on the function argument. For such programs state of the art solvers can easily provide the cost functions, by solving the system of recurrence relations provided by the SRA framework. Table 2.1 shows the benchmarks used in this comparison, their execution behaviour in relation to each function's parameters, and the cost function inferred.

\subsubsection{Experimental method.}

Hardware energy readings were obtained by repeatedly executing a benchmark function over a 0.5 second period, $T$, collecting a set of power samples, $P$, whilst counting the number of executions, $N_{\mathrm{fn}}$. From this, the energy of a single function call, 
Table 2.2: Actual and estimated energy consumption for the fact $(\mathrm{N})$ function over a range of $N$.

\begin{tabular}{|c|c|c|c|c|c|c|}
\hline \multirow{2}{*}{$\begin{array}{c}\text { SRA cost } \\
\text { function }(n J)\end{array}$} & \multirow{2}{*}{$N$} & \multirow{2}{*}{$\begin{array}{r}\text { HW } \\
\text { measured } \\
\text { energy }(n J)\end{array}$} & \multicolumn{2}{|c|}{ Model energy (nJ) } & \multicolumn{2}{|c|}{ Error vs. HW } \\
\hline & & & ISS & SRA & ISS & SRA \\
\hline \multirow{7}{*}{$26.0 N+19.4$} & 1 & 53.1 & 62.8 & 45.3 & 1.18 & 0.85 \\
\hline & 2 & 78.0 & 83.8 & 71.3 & 1.07 & 0.91 \\
\hline & 4 & 127.7 & 125.7 & 123.1 & 0.98 & 0.96 \\
\hline & 8 & 227.1 & 209.6 & 226.8 & 0.92 & 1.00 \\
\hline & 16 & 426.0 & 377.4 & 434.2 & 0.89 & 1.02 \\
\hline & 32 & 823.8 & 713.4 & 849.0 & 0.87 & 1.03 \\
\hline & 64 & 1690.5 & 1387.0 & 1678.4 & 0.82 & 0.99 \\
\hline
\end{tabular}

$E_{\mathrm{fn}}=\frac{\operatorname{mean}(P) \times T}{N_{\mathrm{fn}}}$ is calculated. This was performed using a similar method to the collection of energy model data described in Section 2.4, but was performed on separate hardware so as to de-couple modeling from testing.

ISS modeling involved simulating the same function a smaller number of times than on the hardware in order to keep simulation time adequately low. The instruction statistics were then processed in order to produce an energy figure, and then that figure divided by $N_{\text {fn }}$ was used during ISS in order to extract the energy of a single call. The ISS modeling framework currently has a less efficient test loop than the hardware, potentially reducing accuracy for very short function calls. Similarly, if too few function calls are made during the simulation due to a long-executing function, overrun in the test time may skew low-level energy figures.

Static resource usage analysis was performed by evaluating the produced cost function for a given benchmark with respect to the input arguments, immediately providing the energy cost of a single function call.

\subsubsection{Results.}

Table 2.2 provides an example of test data for the fact (factorial) function. The hardware (HW), low-level Instruction Set Simulation model (ISS), and Static Resource Analysis (SRA) model energy figures are compared. The relative errors of ISS and SRA are compared with respect to the HW energy and normalised as such. The cost function provided for this particular example demonstrates the relationship between the input parameter, $N$, and the SRA estimate of such a call. This, together with data for a number of further benchmarks are presented in graph form in Figure 2.7.

In Figure 2.7, hardware measured energy is compared directly to ISS and SRA energy predictions for the set of four benchmarks. The relative errors are also plotted. In all cases, the ISS model is seen to improve in accuracy as the input parameter $N$ increases, in line with the expected inaccuracies arising from inefficiencies in the mod- 

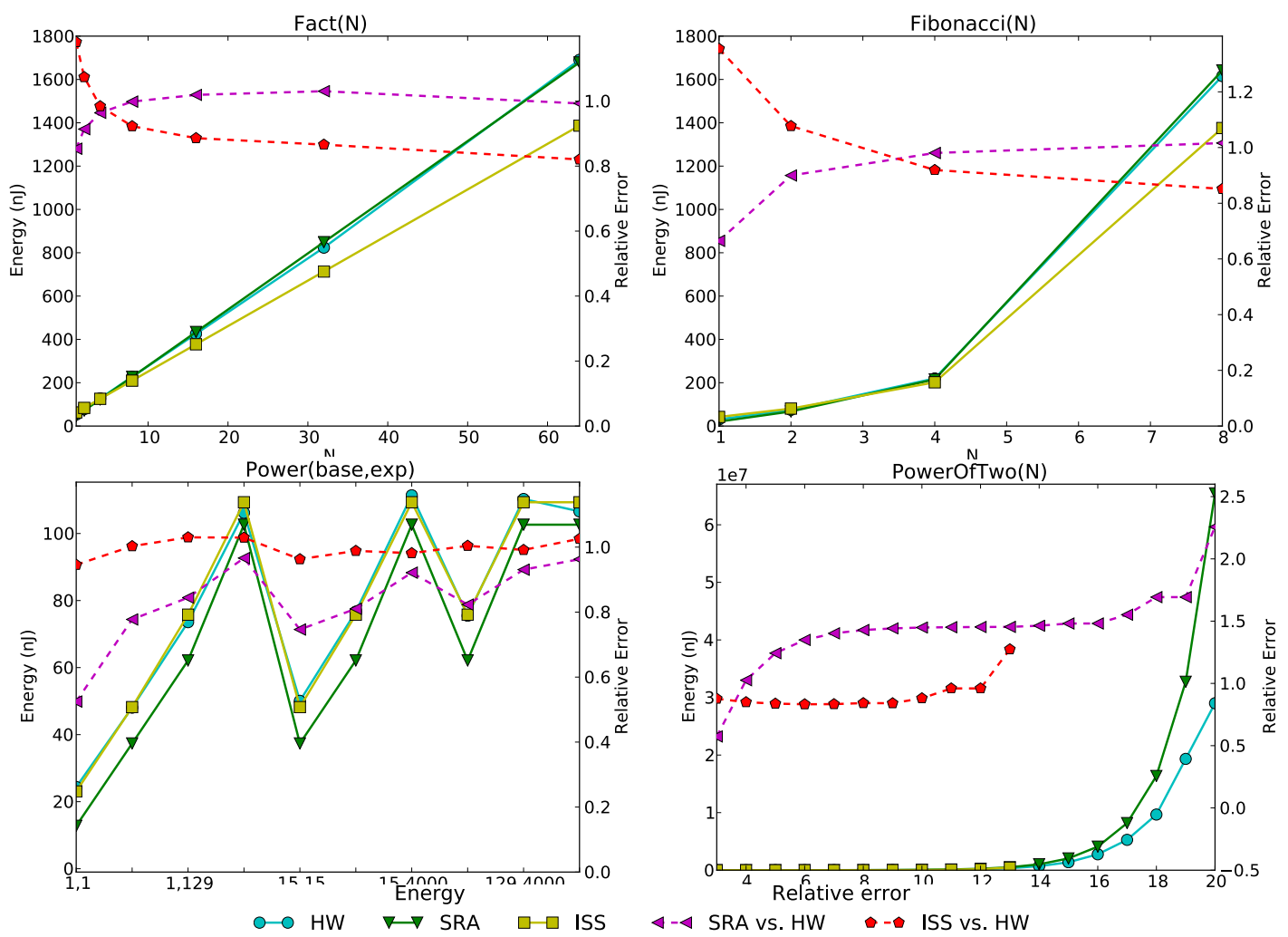

Figure 2.7: Hardware energy, estimations and relative errors for (starting top-left, moving clock-wise) fact, fibonacci, poweroftwo and power.

eling loop used in simulation, as described in the previous subsection. In the case of the poweroftwo function, time limitations prevent the ISS model from approximating the function above $N=13$, approaching which the error begins to increase markedly. The power function behaves in a similar way and demonstrates the relationship between multiple input arguments.

The CiaoPP SRA model does not suffer the same deficiencies, although it does incur a greater underestimation of energy for small values of $N$. The HW measurements unavoidably contain some loop code beyond the target function being examined and small $N$ values will increase the effects of this in the measurement. ISS in fact models this inefficiency directly, whereas SRA does not, hence the roughly symmetrical relative errors for the two models, particularly in the fact and fibonacci cases.

Both approaches are reliant on the same underlying instruction energy figures. Given that some instructions are not directly profiled and, instead, given an average value, accuracy is reduced when the distribution of instructions in a given program is such that the number of profiled instructions is low.

Overall, these results demonstrate both models' capabilities to estimate energy, with encouraging accuracy that can be improved upon. Further, the SRA approach is less restrictive, particularly in situations where simulation time might be prohibitively 
long.

\subsection{Analysis at the LLVM IR Level}

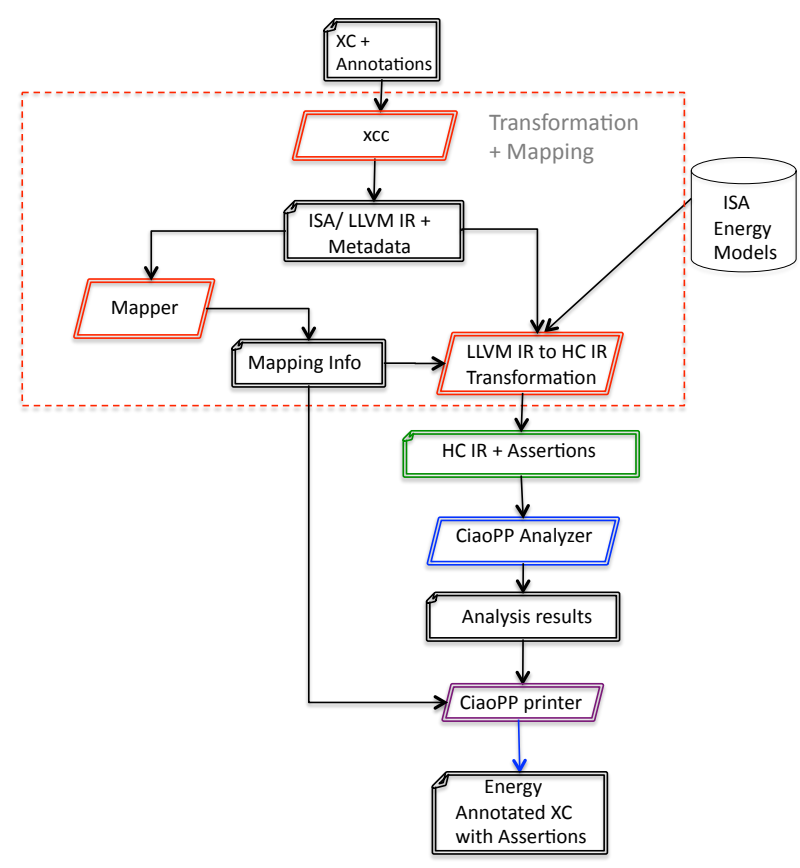

Figure 2.8: An overview of the analysis at the LLVM IR level using ISA models.

An overview of the proposed analysis system at the LLVM IR level using models at the ISA level is depicted in Figure 2.8 which is an another realization of the general analysis framework in Figure 2.1. Similar to ISA realization, the system takes as input an XC source program that can (optionally) contain assertions (used to provide useful hints and information to the analyzer), from which a Transformation and Mapping process (dotted red box) generates first its associated LLVM IR using the xcc compiler. Then, a transformation from LLVM IR into HC IR is performed (explained in Section 2.6.1) obtaining the intermediate representation (green box) that is supplied to the CiaoPP analyzer. This representation includes assertions that express the energy consumed by the LLVM IR blocks, generated from the information produced by the mapper tool (as explained in Section 2.6.2). The CiaoPP analyzer (blue box, described in Section 2.6.3) takes the HC IR, together with the assertions which express the energy consumed by LLVM IR blocks, and possibly some additional (trusted) information, and processes them, producing the analysis results, which are expressed also using assertions. Based on the procedural interpretation of these HC IR programs and the resource-related information contained in the assertions, the resource analysis 
can infer static bounds on the energy consumption of the HC IR programs that are applicable to the original LLVM IR and, hence, to their corresponding XC programs. The analysis results include energy consumption information expressed as functions on data sizes for the whole program and for all the procedures and functions in it. Such results are then processed by the CiaoPP printer (purple box) which presents the information to the program developer in a user-friendly format.

\subsubsection{LLVM IR to HC IR Transformation}

In this section we describe the LLVM IR to HC IR transformation that we have developed in order to achieve the complete analysis system at the LLVM IR level proposed in the chapter (as already mentioned in the overview given in Section 2.6 and depicted in Figure 2.8). The transformation ensures that the program information relevant to resource usage is preserved, so that the energy consumption functions of the HC IR programs inferred by the resource analysis are applicable to the original LLVM IR programs.

The transformation also passes energy values for the LLVM IR level for different programs based on the ISA/LLVM IR mapping information that express the energy consumed by the LLVM IR blocks, as explained in Section 2.6.2. Such information is represented by means of trust assertions (in the Ciao assertion language [40]) that are included in the HC IR. In general, trust assertions can be used to provide information about the program and its constituent parts (e.g., individual instructions or whole procedures or functions) to be trusted by the analysis system, i.e., they provide base information assumed to be true by the inference mechanism of the analysis in order to propagate it throughout the program and obtain information for the rest of its constituent parts.

LLVM IR programs are expressed using typed assembly-like instructions. Each function is in SSA form, represented as a sequence of basic blocks. Each basic block is a sequence of LLVM IR instructions that are guaranteed to be executed in the same order. Each block ends in either a branching or a return instruction. In order to transform an LLVM IR program into the HC IR, we follow a similar approach as in a previous ISA-level transformation explored in Section 2.5.1. However, the LLVM IR includes an additional type transformation as well as better memory modeling.

The following subsections describe the main aspects of the transformation.

\subsubsection{Inferring Block Arguments}

As described before, a block in the HC IR has an entry point (head) with input/output parameters, and a body containing a sequence of steps (here, representations of LLVM IR instructions). Since the scope of the variables in LLVM IR blocks is at the function level, the blocks are not required to pass parameters while making jumps to other blocks. Thus, in order to represent LLVM IR blocks as HC IR blocks, we need to infer input/output parameters for each block as described in 2.5.1.1. 
Note that unlike ISA, the LLVM IR is in SSA form at the function level, which means that blocks may have $\phi$ nodes which are created while transforming the program into SSA form. A $\phi$ node is essentially a function defining a new variable by selecting one of the multiple instances of the same variable coming from multiple predecessor blocks:

$$
x=\phi\left(x_{1}, x_{2}, \ldots, x_{n}\right)
$$

def and ref for this instruction are $\{x\}$ and $\left\{x_{1}, x_{2}, \ldots, x_{n}\right\}$ respectively. An interesting feature of our approach is that $\phi$ nodes are not needed. Once the input/output parameters are inferred for each block as explained above, a post-process gets rid of all $\phi$ nodes by modifying block input arguments in such a way that blocks receive $x$ directly as an input and an appropriate $x_{i}$ is passed by the call site. This will be illustrated later in Section 2.6.1.3.

Consider the example in Figure 2.10 (left), where the LLVM IR block looptest is defined. The body of the block reads from 2 variables without previously defining them in the same block. The fixpoint analysis would yield:

$$
\operatorname{params}_{\text {in }}(\text { looptest })=\{\text { Arr }, I\}
$$

which is used to construct the HC IR representation of the looptest block shown in Figure 2.10 (right), line 3.

\subsubsection{Translating LLVM IR Types into HC IR Types}

LLVM IR is a typed representation which allows retaining much more of the (source) program information than the ISA representation (e.g., types defining compound data structures). As already mentioned, this enables a more direct analysis as well as mapping of the analysis information back to source level. Thus, we define a mechanism to translate LLVM IR types into their counterparts in HC IR.

The LLVM type system defines primitive and derived types. The primitive types are the fundamental building blocks of the type system. Primitive types include label, void, integer, character, floating point, x86mmx, and metadata. The $x 86 m m x$ type represents a value held in an MMX register on an X86 machine and the metadata type represents embedded metadata. The derived types are created from primitive types or other derived types. They include array, function, pointer, structure, vector, opaque. Since the XCore platform supports neither pointers nor floating point data types, the LLVM IR code generated from XC programs uses only a subset of the LLVM types.

At the HC IR level we use regular types, one of the type systems supported by CiaoPP [42]. Translating LLVM IR primitive types into regular types is straightforward. The integer and character types are abstracted as num regular type, whereas the label, void, and metadata types are represented as atm (atoms).

For derived types, corresponding non-primitive regular types are constructed during the transformation phase. Supporting non-primitive types is important because it enables the analysis to infer energy consumption functions that depend on the sizes 
of internal parts of complex data structures. The array, vector, and structure types are represented as follows:

$$
\begin{aligned}
\text { array_type } & \rightarrow(\text { nested }) \text { list } \\
\text { vector_type } & \rightarrow(\text { nested }) \text { list } \\
\text { structure_type } & \rightarrow \text { functor_term }
\end{aligned}
$$

Both the array and vector types are represented by the list type in CiaoPP which is a special case of compound term. The type of the elements of such lists can be again a primitive or a derived type. The structure type is represented by a compound term which is composed of an atom (called the functor, which gives a name to the structure) and a number of arguments, which are again either primitive or derived types. LLVM also introduces pointer types in the intermediate representation, even if the front-end language does not support them (as in the case of XC, as mentioned before). Pointers are used in the pass-by-reference mechanism for arguments, in memory allocations in alloca blocks, and in memory load and store operations. The types of these pointer variables in the HC IR are the same as the types of the data these pointers point to.

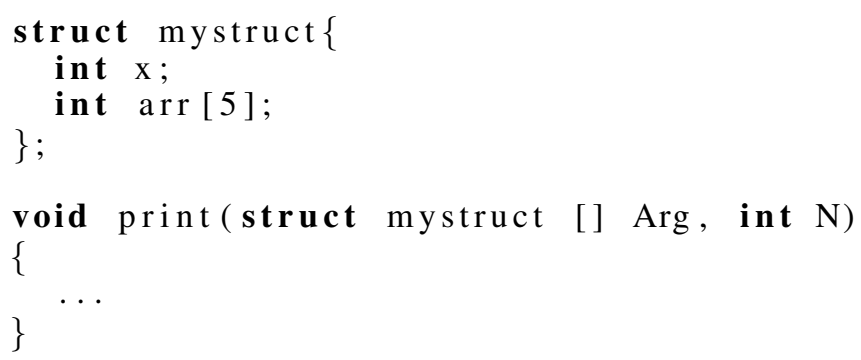

Figure 2.9: An XC program and its type transformation into HC IR.

Consider for example the types in the XC program shown in Figure 2.9. The type of argument Arg of the print function is an array of mystruct elements. mystruct is further composed of an integer and an array of integers. The LLVM IR code generated by xcc for the function signature print in Figure 2.9 (left) is:

$$
\text { define void @ print }([0 \times\{i 32,[5 \times i 32]\}] * \text { noalias nocapture })
$$

The function argument type in the LLVM IR $([0 \times\{i 32,[5 \times i 32]\}])$ is the typed representation of the argument $\mathrm{Arg}$ to the function in the XC program. It represents an array of arbitrary length with elements of $\{i 32,[5 \times i 32]\}$ structure type which is further composed of an $i 32$ integer type and a [5×i32] array type, i.e., an array of 5 elements of $i 32$ integer type. ${ }^{1}$

This type is represented in the HC IR using the set of regular types illustrated in Figure 2.9 (right). The regular type array1, is a list of struct elements (which can also be simply written as arrayl $:=$ list (struct)). Each struct type element is represented as a functor mystruct/2 where the first argument is a num and the second is another list type array 2 . The type array 2 is defined to be a list of num (which, again, can also be simply written as array $2:=$ list (num)).

\footnotetext{
${ }^{1}[0 \times i 32]$ specifies an arbitrary length array of $i 32$ integer type elements.
} 
indicates that if the $n t h(I, L, E l e m)$ predicate (representing the getelementptr LLVM IR instruction) is called with $I$ and $L$ bound to an integer and a list of numbers respectively, and Elem an unbound variable (precondition field ":"), then, after the successful completion of the call (postcondition field "=>"), Elem is an integer number and the lower and upper bounds on its size are equal to the lower and upper bounds on the sizes of the elements of the list $L$. The sizes of the arguments to $n t h / 3$ are expressed using the property rsize in the assertion language. The lower and upper bounds on the length of the list $L$ are $L L$ and $L U$ respectively. Similarly, the lower and upper bounds on the elements of the list are $E L$ and $E U$ respectively, which are also the bounds for Elem. The resource property (global computational properties field + ) expresses that the energy consumption for the instruction is an average value (1215439 nano-joules ${ }^{2}$ ).

The branching instructions in LLVM IR are transformed into calls to target blocks in HC IR. For example, the branching instruction at line 6 in Figure 2.10 (left), which jumps to one of the two blocks loopbody or loopend based on the Boolean variable $Z c m p$, is transformed into a call to a predicate with two clauses (line 5 in Figure 2.10 (right)). The name of the predicate is the concatenation of the names of the two LLVM IR blocks mentioned above. The two clauses of the predicate defined at lines 8-13 and 14-15 in Figure 2.10 (right) represent the LLVM IR blocks loopbody and loopend respectively. The test on the conditional variable is placed in both clauses to preserve the semantics of the conditional branch.

\subsubsection{Obtaining the Energy Consumption of LLVM IR Blocks}

Our approach requires producing assertions that express the energy consumed by each call to an LLVM IR block (or parts of it) when it is executed. To achieve this we take as starting point the energy consumption information available from an existing XS1L ISA Energy Model produced in our work of ISA level analysis [62] (also detailed in Section 2.4) using the techniques described in [51]. We refer the reader to [51] for a detailed study of the energy consumption behaviour of the XS1-L architecture, containing a description of the test and measurement process along with the construction and full evaluation of such model. In the experiments performed in this chapter a single, constant energy value is assigned to each instruction in the ISA based on this model.

A mechanism is then needed to propagate such ISA-level energy information up to the LLVM IR level and obtain energy values for LLVM IR blocks. A set of mapping techniques serve this purpose by creating a fine-grained mapping between segments of ISA instructions and LLVM IR code segments, in order to enable the energy characterization of each LLVM IR instruction in a program, by aggregating the energy consumption of the ISA instructions mapped to it. Then, the energy value assigned to each LLVM IR block is obtained by aggregating the energy consumption of all its LLVM IR instructions. The mapping is done by using the debug mechanism where the debug information, preserved during the lowering phase of the compilation from

\footnotetext{
${ }^{2} \mathrm{~nJ}, 10^{-9}$ joules
} 
LLVM IR to ISA, is used to track ISA instructions against LLVM IR instructions. A full description and formalization of the mapping techniques is given in [31].

\subsubsection{Resource Analysis with CiaoPP}

The analysis of HC IR code coming from LLVM IR is performed in a similar fashion as it is done for ISA code in Section 2.5.2. We provide assertions for each HC IR block, expressing the energy consumed by the corresponding LLVM IR block, that is derived from the energy model, as explained in Section 2.6.2. Based on this information, the global static analysis can then infer bounds on the resource usage of the whole program (as well as procedures and functions in it) as functions of input data sizes.

Consider the example in Figure 2.10 (right). Let $P_{e}$ denote the energy consumption function for a predicate $P$ in the HC IR representation (set of blocks with the same name). Let $c_{b}$ represent the energy cost of an LLVM IR block $b$. Then, the inferred equations for the HC IR blocks in Figure 2.10 (right) are:

$$
\begin{aligned}
& \text { alloca }_{e}(N, \text { Arr })=c_{\text {alloca }}+\text { looptest }_{e}(N, \text { Arr }) \\
& \text { looptest }_{e}(N, A r r)=c_{\text {looptest }}+\text { loopbody_loopend }_{e}(0 \neq N, N, A r r) \\
& \text { loopbody_loopend }_{e}(B, N, A r r)=\left\{\begin{array}{l}
\text { looptest }_{e}(N-1, A r r) \text { if } B \text { is true } \\
+c_{\text {loopbody }} \\
c_{\text {loopend }}
\end{array}\right.
\end{aligned}
$$

If we assume (for simplicity of exposition) that each LLVM IR block has unitary cost, i.e., $c_{b}=1$ for all LLVM IR blocks $b$, solving the above recurrence equations, we obtain the energy consumed by alloca as a function of its input data size $(N)$ :

$$
\operatorname{alloca}_{e}(N, A r r)=2 \times N+3
$$

As explained before in Section 2.5.3, the use of average energy values in the model implies that the energy function for the whole program inferred by the upper-bound resource analysis is an approximation of the actual upper bound that can possibly be below it. We discuss this in more detail in Chapter 3.

\subsection{Experimental Evaluation}

We have performed an experimental evaluation of our techniques on a number of selected benchmarks. Power measurement data was collected for the XCore platform by using appropriately instrumented power supplies, a power-sense chip, and an embedded system for controlling the measurements and collecting the power data. Details about the power monitoring setup used to run our benchmarks and measure their energy consumption can be found in [51]. The main goal of our experiments was to shed light on the trade-offs implied by performing the analysis at the ISA level (without using complex mechanisms for propagating type information and representing memory) 
and at the LLVM level using models defined at the ISA level together with a mapping mechanism.

There are two groups of benchmarks that we have used in our experimental study. The first group is composed of four small recursive numerical programs that have a variety of user defined functions, arguments, and calling patterns (first four benchmarks in Table 2.4). These benchmarks only operate over primitive data types and do not involve any structured types. The second group of benchmarks (the last five benchmarks in Table 2.4) differs from the first group in the sense that they all involve structured types. These are recursive or iterative.

The second group of benchmarks includes two filter benchmarks namely Biquad and Finite Impulse Response (FIR). A filter program attenuates or amplifies one specific frequency range of a given input signal. The $\mathrm{fir}(\mathrm{N})$ benchmark computes the inner-product of two vectors: a vector of input samples, and a vector of coefficients. The more coefficients, the higher the fidelity, and the lower the frequencies that can be filtered. On the other hand, the Biquad benchmark is an equaliser running Biquad filtering. An equaliser takes a signal and attenuates/amplifies different frequency bands. In the case of an audio signal, such as in a speaker or microphone, this corrects the frequency response. The biquad (N) benchmark uses a cascade of Biquad filters where each filter attenuates or amplifies one specific frequency range. The energy consumed depends on the number of banks N, typically between 3 and 30 for an audio equaliser. A higher number of banks enables a designer to create more precise frequency response curves.

None of the XC benchmarks contain any assertions that provide information to help the analyzer. Table 2.3 shows detailed experimental results. Column SA energy function shows the energy consumption functions, which depend on input data sizes, inferred for each program by the static analyses performed at the ISA and LLVM IR levels (denoted with subscripts isa and $l l v m$ respectively). We can see that the analysis is able to infer different kinds of functions (polynomial, exponential, etc.). Column HW shows the actual energy consumption in nano-joules measured on the hardware corresponding to the execution of the programs with input data of different sizes (shown in column Input Data Size). Estimated presents the energy consumption estimated by static analysis. This is obtained by evaluating the functions in column SA energy function for the input data sizes in column Input Data Size. The value N/A in such column means that the analysis has not been able to infer any useful energy consumption function and, thus, no estimated value is obtained. Column Err vs. HW shows the error of the values estimated by the static analysis with respect to the actual energy consumption measured on the hardware, calculated as follows: Err vs. HW $=\left(\frac{\mathbf{L L V M}(\text { or } \mathbf{I S A})-\mathbf{H W}}{\mathbf{H W}} \times 100\right) \%$. Finally, the last column shows the ratio between the estimations of the analysis at the ISA and LLVM IR levels.

\footnotetext{
${ }^{3}$ It uses mathematical functions $f i b$ and lucas, a function expansion would yield: $F i b_{i s a}(N)=34.87 \times 1.62^{N}+10.8 \times(-0.62)^{N}-30$ $F_{i b v m}(N)=40.13 \times 1.62^{N}+11.1 \times(-0.62)^{N}-35.65$

${ }^{4} \operatorname{Lucas}(n)$ satisfy the recurrence relation $L_{n}=L_{n-1}+L_{n-2}$ with $L_{1}=1, L_{2}=3$
} 
CHAPTER 2. MULTI-LANGUAGE ENERGY CONSUMPTION ANALYSIS AND

MODELING FRAMEWORK

\begin{tabular}{|c|c|c|c|c|c|c|c|}
\hline \multirow{2}{*}{$\begin{array}{r}\text { SA energy } \\
\text { function }(\mathbf{n J})\end{array}$} & \multirow{2}{*}{$\begin{array}{r}\text { Input } \\
\text { Size }\end{array}$} & \multirow[t]{2}{*}{ HW (nJ) } & \multicolumn{2}{|c|}{ Estimated (nJ) } & \multicolumn{2}{|c|}{ Err vs. HW\% } & \multirow{2}{*}{$\begin{array}{l}\text { isa/ } \\
\text { llvm } \\
\end{array}$} \\
\hline & & & llvm & isa & llvm & isa & \\
\hline \multirow{4}{*}{$\begin{array}{c}\text { Fact }_{\text {isa }}(N)= \\
24.26 N+18.43 \\
\operatorname{Fact}_{l v m}(N)= \\
27.03 N+21.28\end{array}$} & $\mathrm{~N}=8$ & 227 & 237 & 212 & $\overline{4.6}$ & \begin{tabular}{c|}
-6.4 \\
\end{tabular} & 0.9 \\
\hline & $\mathrm{N}=16$ & 426 & 453 & 406 & 6.5 & -4.5 & 0.9 \\
\hline & $\mathrm{N}=32$ & 824 & 886 & 794 & 7.6 & -3.5 & 0.9 \\
\hline & $\mathrm{N}=64$ & 1690 & 1751 & 1571 & 3.6 & -7.0 & 0.9 \\
\hline \multirow{3}{*}{ 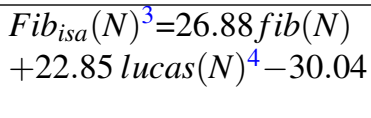 } & $\mathrm{N}=2$ & 75 & 74 & 65 & -1.1 & -12 & $\overline{0.89}$ \\
\hline & $\mathrm{N}=4$ & 219 & 241 & 210 & 10 & -4.1 & 0.87 \\
\hline & $\mathrm{N}=8$ & 1615 & 1853 & 1608 & 14.75 & -0.4 & 0.87 \\
\hline \multirow{2}{*}{$\begin{array}{r}\text { Fib llvm }_{\text {ll }}(N)^{a}=32.5 \text { fib }(N) \\
+25.6 \text { lucas }(N)^{b}-35.6\end{array}$} & $\mathrm{~N}=15$ & $47 \times 10^{3}$ & $54 \times 10^{3}$ & $47 \times 10^{3}$ & 16.47 & 1.2 & 0.87 \\
\hline & $\mathrm{N}=26$ & $9.30 \times 10^{6}$ & $10.9 \times 10^{6}$ & $9.5 \times 10^{6}$ & 17.3 & 1.74 & 0.87 \\
\hline \multirow{3}{*}{$\begin{array}{l}\operatorname{Sqr}_{\text {isa }}(N)= \\
\quad 8.6 N^{2}+48.7 N+15.6\end{array}$} & $\mathrm{~N}=9$ & 1242 & 1302 & 1148 & 4.8 & -7.5 & 0.88 \\
\hline & $\mathrm{N}=27$ & 8135 & 8734 & 7579 & 7.4 & -6.8 & 0.87 \\
\hline & $\mathrm{N}=73$ & $52 \times 10^{3}$ & $57 \times 10^{3}$ & $49 \times 10^{3}$ & 8.5 & -6.5 & 0.86 \\
\hline \multirow{3}{*}{$\begin{array}{l}\operatorname{Sqr}_{l l v m}(N)= \\
\quad 10 N^{2}+53 N+15.6\end{array}$} & $\mathrm{~N}=144$ & $19.7 \times 10^{4}$ & $21.4 \times 10^{4}$ & $18.4 \times 10^{4}$ & 8.89 & -6.4 & 0.86 \\
\hline & $\mathrm{N}=234$ & $51 \times 10^{4}$ & $56 \times 10^{4}$ & $48 \times 10^{4}$ & 9.61 & -5.8 & 0.86 \\
\hline & $\mathrm{N}=360$ & $11.9 \times 10^{5}$ & $13 \times 10^{5}$ & $11.2 \times 10^{5}$ & 10.49 & -5.1 & 0.86 \\
\hline \multirow{5}{*}{$\begin{array}{c}\text { PowerOfTwo } \text { isa }_{\text {isa }}(N)= \\
41.5 \times 2^{N}-25.9 \\
\text { PowerOfTwollvm }(N)= \\
46.8 \times 2^{N}-29.9\end{array}$} & $\mathrm{~N}=3$ & 326 & 344 & 3.6 & 5.7 & -6.0 & 0.89 \\
\hline & $\mathrm{N}=6$ & 2729 & 2965 & 2631 & 8.7 & 3.6 & 0.89 \\
\hline & $\mathrm{N}=9$ & $21.9 \times 10^{3}$ & $23.9 \times 10^{3}$ & $21.2 \times 10^{3}$ & 9 & 3.3 & $\overline{0.89}$ \\
\hline & $\mathrm{N}=12$ & $17.6 \times 10^{4}$ & $19.1 \times 10^{4}$ & $17 \times 10^{4}$ & 9 & -3.3 & 0.89 \\
\hline & $\mathrm{N}=15$ & $13.8 \times 10^{5}$ & $15.3 \times 10^{5}$ & $13.6 \times 10^{5}$ & 11 & -1.5 & 0.89 \\
\hline \multirow{5}{*}{$\begin{array}{l}\text { reverse }_{\text {llvm }}(N)= \\
19.47 N+69.33\end{array}$} & $\mathrm{~N}=57$ & 1138 & 1179 & N/A & 3.60 & N/A & N/A \\
\hline & $\mathrm{N}=160$ & 3125 & 3185 & N/A & 1.91 & N/A & N/A \\
\hline & $\mathrm{N}=320$ & 6189 & 6301 & N/A & 1.82 & N/A & N/A \\
\hline & $\mathrm{N}=720$ & 13848 & 14092 & N/A & 1.76 & N/A & N/A \\
\hline & $\mathrm{N}=1280$ & 24634 & 24998 & N/A & 1.48 & N/A & N/A \\
\hline \multirow{5}{*}{$\begin{array}{r}\text { matmult }_{\text {llvm }}(N)= \\
42.47 N^{3}+68.85 N^{2}+ \\
49.9 N+24.22\end{array}$} & $\mathrm{~N}=5$ & 7453 & 7569 & N/A & -2 & N/A & N/A \\
\hline & $\mathrm{N}=15$ & $15.8 \times 10^{4}$ & $15.9 \times 10^{4}$ & N/A & 1.03 & N/A & N/A \\
\hline & $\mathrm{N}=20$ & $36.3 \times 10^{4}$ & $36.8 \times 10^{4}$ & N/A & 1.51 & N/A & N/A \\
\hline & $\mathrm{N}=25$ & $69.5 \times 10^{4}$ & $70.8 \times 10^{4}$ & N/A & 1.77 & N/A & N/A \\
\hline & $\mathrm{N}=31$ & $13.1 \times 10^{5}$ & $13.3 \times 10^{5}$ & N/A & 1.98 & N/A & N/A \\
\hline \multirow{4}{*}{$\begin{array}{l}\operatorname{concat}_{l l v m}(N, M)= \\
65.7 N+65.7 M+137\end{array}$} & $\begin{array}{r}\mathrm{N}=131 \\
\mathrm{M}=69\end{array}$ & $14.5 \times 10^{3}$ & $13.2 \times 10^{3}$ & $\overline{\mathrm{N} / \mathrm{A}}$ & 8.65 & N/A & N/A \\
\hline & $\begin{array}{l}\mathrm{N}=170 \\
\mathrm{M}=182\end{array}$ & $\begin{array}{r}25.44 \times \\
10^{3}\end{array}$ & $23.3 \times 10^{3}$ & N/A & 8.60 & N/A & N/A \\
\hline & $\begin{array}{r}\mathrm{N}=188 ; \\
\mathrm{M}=2\end{array}$ & $13.8 \times 10^{3}$ & $12.6 \times 10^{3}$ & N/A & 8.59 & N/A & N/A \\
\hline & $\begin{array}{r}\mathrm{N}=13 ; \\
\mathrm{M}=134\end{array}$ & $10.7 \times 10^{3}$ & $9.79 \times 10^{3}$ & N/A & 8.74 & N/A & N/A \\
\hline \multirow{4}{*}{$\begin{array}{r}\operatorname{biquad}_{l l v m}(N)= \\
157 N+51.7\end{array}$} & $\mathrm{~N}=5$ & 871 & 836 & N/A & -4 & N/A & N/A \\
\hline & $\mathrm{N}=7$ & 1187 & 1151 & N/A & -3.1 & N/A & N/A \\
\hline & $\mathrm{N}=10$ & 1660 & 1622 & N/A & -2.3 & N/A & N/A \\
\hline & $\mathrm{N}=14$ & 2290 & 2250 & N/A & -1.7 & N/A & N/A \\
\hline \multirow{4}{*}{$\begin{array}{l}\operatorname{fir}_{l l v m}(N)= \\
\quad 31.8 N+137\end{array}$} & $\mathrm{~N}=85$ & 2999 & 2839 & N/A & -5.3 & N/A & N/A \\
\hline & $\mathrm{N}=97$ & 3404 & 3221 & N/A & -5.4 & N/A & N/A \\
\hline & $\mathrm{N}=109$ & 3812 & 3602 & N/A & -5.5 & N/A & N/A \\
\hline & $\mathrm{N}=121$ & 4227 & 3984 & N/A & -5.7 & N/A & N/A \\
\hline
\end{tabular}

Table 2.3: Comparison of the accuracy of energy analyses at the LLVM IR and ISA levels. 
Table 2.4 shows a summary of results. The first two columns show the name and short description of the benchmarks. The columns under Err vs. HW show the average error obtained from the values given in Table 2.3 for different input data sizes. The last row of the table shows the average error over the number of benchmarks analyzed at each level.

\begin{tabular}{|l|l|r|r|c|}
\hline \multirow{2}{*}{ Program } & Description & \multicolumn{2}{|c|}{ Err vs. HW } & isa/ \\
\cline { 3 - 4 } & & llvm & isa & llvm \\
\hline \hline fact $(\mathrm{N})$ & Calculates N! & $5.6 \%$ & $5.3 \%$ & 0.89 \\
\hline fibonacci $(\mathrm{N})$ & Nth Fibonacci number & $11.9 \%$ & $4 \%$ & 0.87 \\
\hline sqr $(\mathrm{N})$ & Computes $N^{2}$ performing additions & $9.3 \%$ & $3.1 \%$ & 0.86 \\
\hline pow_of two $(\mathrm{N})$ & Calculates $2^{N}$ without multiplication & $9.4 \%$ & $3.3 \%$ & 0.89 \\
\hline Average & & $\mathbf{9 \%}$ & $\mathbf{3 . 9 \%}$ & $\mathbf{0 . 9 2}$ \\
\hline \hline reverse $(\mathrm{N}, \mathrm{M})$ & Reverses an array & $2.18 \%$ & N/A & N/A \\
\hline concat $(\mathrm{N}, \mathrm{M})$ & Concatenation of arrays & $8.71 \%$ & N/A & N/A \\
\hline matmult $(\mathrm{N}, \mathrm{M})$ & Matrix multiplication & $1.47 \%$ & N/A & N/A \\
\hline fir $(\mathrm{N})$ & Finite Impulse Response filter & $5.47 \%$ & N/A & N/A \\
\hline biquad $(\mathrm{N})$ & Biquad equaliser & $3.70 \%$ & N/A & N/A \\
\hline Average & & $\mathbf{3 . 0 \%}$ & N/A & N/A \\
\hline \hline Overall average & & $\mathbf{6 . 4 \%}$ & $\mathbf{3 . 9 \%}$ & $\mathbf{0 . 9 2}$ \\
\hline
\end{tabular}

Table 2.4: LLVM IR- vs. ISA-level analysis accuracy.

The experimental results show that:

- For the benchmarks in the first group, both the ISA- and LLVM IR-level analyses are able to infer useful energy consumption functions. On average, the analysis performed at either level is reasonably accurate and the relative error between the two analyses at different levels is small. ISA-level estimations are slightly more accurate than the ones at the LLVM IR level (3.9\% vs. 9\% error on average with respect to the actual energy consumption measured on the hardware, respectively). This is because the ISA-level analysis uses very accurate energy models, obtained from measuring directly at the ISA level, whereas at the LLVM IR level, such ISA-level model needs to be propagated up to the LLVM IR level using (approximated) mapping information. This causes a slight loss of accuracy.

- For the second group of benchmarks, the ISA level analysis is not able to infer useful energy functions. This is due to the fact that significant program structure and data type/shape information is lost due to lower-level representations, which sometimes makes the analysis at the ISA level very difficult or impossible. In order to overcome this limitation and improve analysis accuracy, significantly more complex techniques for recovering type information and representing memory in the HC IR would be needed. In contrast, type/shape information is preserved at 
the LLVM IR level, which allows analyzing programs using data structures (e.g., arrays). In particular, all the benchmarks in the second group are analyzed at the LLVM IR level with reasonable accuracy (3\% error on average). In this sense, the LLVM IR-level analysis is more powerful than the one at the ISA level. The analysis is also reasonably efficient, with analysis times of about 5 to 6 seconds on average, despite the naive implementation of the interface with external recurrence equation solvers, which can be improved significantly. The scalability of the analysis follows from the fact that it is compositional and can be performed in a modular way, making use of the Ciao assertion language to store results of previously analyzed modules.

\subsection{Energy Consumption Verification via Static Analy- sis}

In this section we leverage an existing general framework for resource usage verification and specialize it for verifying energy consumption specifications of embedded programs. Such specifications can include both lower and upper bounds on energy usage, and they can express intervals within which energy usage is to be certified to be within such bounds. The bounds of the intervals can be given in general as functions on input data sizes. Our verification system can prove whether such energy usage specifications are met or not, by comparing the specifications with the approximated energy consumption information inferred by the static analysis. It can also infer the particular conditions under which the specifications hold. To this end, these conditions are also expressed as intervals of functions of input data sizes, such that a given specification can be proved for some intervals but disproved for others. The specifications themselves can also include preconditions expressing intervals for input data sizes. We report on a prototype implementation of our approach within the CiaoPP system for the XC language and XS1-L architecture, and illustrate with an example how embedded software developers can use this tool, and in particular for determining values for program parameters that ensure meeting a given energy budget while minimizing the loss in quality of service.

\subsubsection{Introduction}

In an increasing number of applications, particularly those running on devices with limited resources, it is very important and sometimes essential to ensure conformance with respect to specifications expressing non-functional global properties such as energy consumption, maximum execution time, memory usage, or user-defined resources. For example, in a real-time application, a program completing an action later than required is as erroneous as a program not computing the correct answer. The same applies to an embedded application in a battery-operated device (e.g., a portable or implantable medical device, an autonomous space vehicle, or even a mobile phone) if 


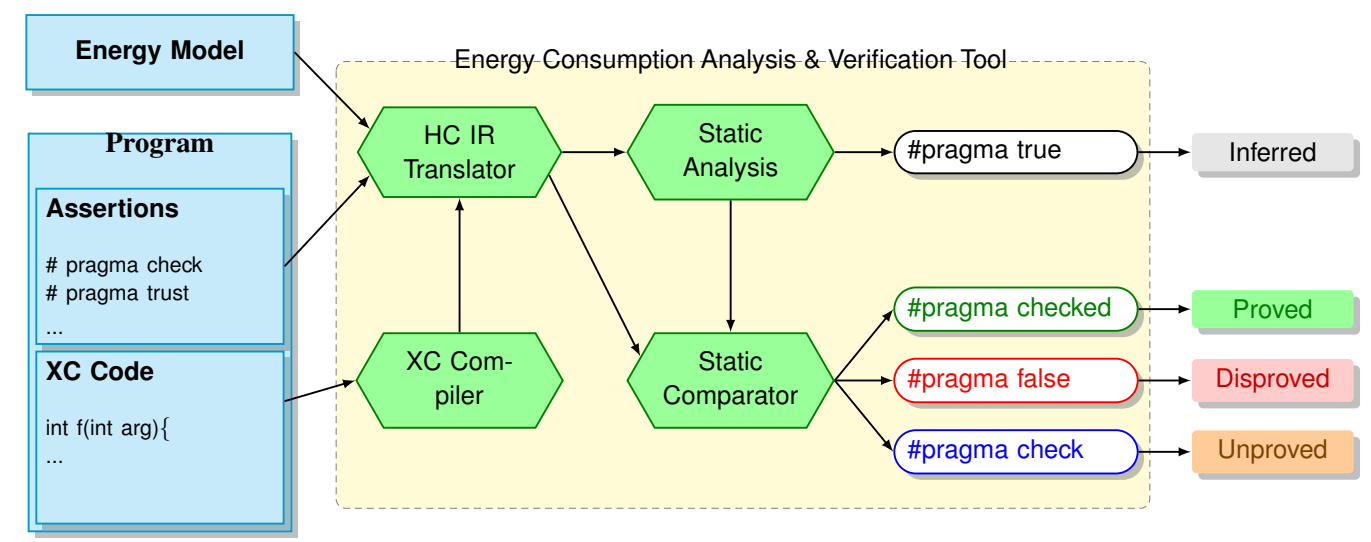

Figure 2.11: Energy consumption verification tool using CiaoPP.

the application makes the device run out of batteries earlier than required, making the whole system useless in practice.

In general, high performance embedded systems must control, react to, and survive in a given environment, and this in turn establishes constraints about the system's performance parameters including energy consumption and reaction times. Therefore, a mechanism is necessary in these systems in order to prove correctness with respect to specifications about such non-functional global properties.

To address this problem we leverage an existing general framework for resource usage verification $[63,64]$, and specialize it for verifying energy consumption specifications of embedded programs. As a case study, we focus on the energy verification of embedded programs written in the XC language [95] and running on the XMOS $\mathrm{XS1}$-L architecture (XC is a high-level C-based programming language that includes extensions for communication, input/output operations, real-time behavior, and concurrency). However, the approach presented here can also be applied to the analysis of other programming languages and architectures. We will illustrate with an example how embedded software developers can use this tool, and in particular for determining values for program parameters that ensure meeting a given energy budget while minimizing the loss in quality of service.

\subsubsection{Overview of the Energy Verification Tool}

In this section we give an overview of the prototype tool for energy consumption verification of XC programs running on the XMOS XS1-L architecture, which we have implemented within the CiaoPP system [42]. As described in previous sections we differentiate between the input language, which can be XC source, LLVM IR, or Instruction Set Architecture (ISA) code, and the intermediate semantic program representation that the CiaoPP core components (e.g., the analyzer) take as input.

Figure 2.11 shows an overview diagram of the architecture of the prototype tool we have developed. Hexagons represent different tool components and arrows indicate the communication paths among them. 
The tool takes as input an XC source program (left part of Figure 2.11) that can optionally contain assertions in a C-style syntax. As we will see later, such assertions are translated into Ciao assertions, the internal representation used in the Ciao/CiaoPP system.

The energy specifications that the tool will try to prove or disprove are expressed by means of assertions with check status. These specifications can include both lower and upper bounds on energy usage, and they can express intervals within which energy usage is to be certified to be within such bounds. The bounds of the intervals can be given in general as functions on input data sizes. Our tool can prove whether such energy usage specifications are met or not. It can also infer the particular conditions under which the specifications hold. To this end, these conditions are also expressed as intervals of functions of input data sizes, such that a given specification can be proved for some intervals but disproved for others.

In addition, assertions can also express trusted information such as the energy usage of procedures that are not developed yet, or useful hints and information to the tool. In general, assertions with status trust can be used to provide information about the program and its constituent parts (e.g., individual instructions or whole procedures or functions) to be trusted by the analysis system, i.e., they provide base information assumed to be true by the inference mechanism of the analysis in order to propagate it throughout the program and obtain information for the rest of its constituent parts.

The associated ISA and/or LLVM IR representations of the XC program are generated using the xcc compiler. Such representations include useful metadata. The $H C$ IR translator component (described in Section 2.6.1) produces the internal representation used by the tool, HC IR, which includes the program and possibly specifications and/or trusted information (expressed in the Ciao assertion language [82, 40]). The tool performs the transformation of specifications (and trusted information) written as C-like assertions into the Ciao assertion language.

Then, following the approach described in Sections 2.5 and 2.6, the CiaoPP parametric static resource usage analyzer takes the HC IR, together with the assertions which express the energy consumed by LLVM IR blocks and/or individual ISA instructions, and possibly some additional (trusted) information, and processes them, producing the analysis results, which are expressed also using Ciao assertions. Such results include energy usage functions (which depend on input data sizes) for each block in the HC IR (i.e., for the whole program and for all the procedures and functions in it.). Analysis results are given using the assertion language, to ensure interoperability and make them understandable by the programmer.

The verification of energy specifications is performed by a specialized component which compares the energy specifications with the (safe) approximated information inferred by the static resource analysis. Such component is based on a previous work on general resource usage verification presented in [63, 64], where the authors extended the criteria of correctness as the conformance of a program to a specification expressing non-functional global properties, such as upper and lower bounds on execution time, memory, energy, or user defined resources, given as functions on input 
data sizes. We also defined an abstract semantics for resource usage properties and operations to compare the (approximated) intended semantics of a program (i.e., the specification) with approximated semantics inferred by static analysis. These operations include the comparison of arithmetic functions (e.g., polynomial, exponential, or logarithmic functions) that may come from the specifications or from the analysis results. As a possible result of the comparison in the output of the tool, either:

1. The original (specification) assertion (i.e., with status check) is included with status checked (resp. false), meaning that the assertion is correct (resp. incorrect) for all input data meeting the precondition of the assertion,

2. the assertion is "split" into two or three assertions with different status (checked, false, or check) whose preconditions include a conjunct expressing that the size of the input data belongs to the interval(s) for which the assertion is correct (status checked), incorrect (status false), or the tool is not able to determine whether the assertion is correct or incorrect (status check), or

3. in the worst case, the assertion is included with status check, meaning that the tool is not able to prove nor to disprove (any part of) it.

If all assertions are checked then the program is verified. Otherwise, for assertions (or parts of them) that get false status, a compile-time error is reported. Even if a program contains no assertions, it can be checked against the assertions contained in the libraries used by the program, potentially catching bugs at compile time. Finally, and most importantly, for assertion (or parts of them) left with status check, the tool can optionally produce a verification warning (also referred to as an "alarm"). In addition, optional run-time checks can also be generated.

\subsubsection{The Assertion Language}

Two aspects of the assertion language are described here: the front-end language in which assertions are written and included in the XC programs to be verified, and the internal language in which such assertions are translated into and passed, together with the HC IR program representation, to the core analysis and verification tools, the Ciao assertion language.

\subsubsection{The Ciao Assertion Language}

We describe here the subset of the Ciao assertion language which allows expressing global "computational" properties and, in particular, resource usage. We refer the reader to $[82,42,40]$ and their references for a full description of this assertion language.

For brevity, we only introduce here the class of pred assertions, which describes a particular predicate and, in general, follows the schema:

$$
\text { :- pred Pred [: Precond }][=>\text { Postcond }][+ \text { Comp-Props }] \text {. }
$$


where Pred is a predicate symbol applied to distinct free variables while Precond and Postcond are logic formulae about execution states. An execution state is defined by variable/value bindings in a given execution step. The assertion indicates that in any call to Pred, if Precond holds in the calling state and the computation of the call succeeds, then Postcond also holds in the success state. Finally, the Comp-Props field is used to describe properties of the whole computation for calls to predicate Pred that meet Precond. In our application Comp-Props are precisely the resource usage properties.

For example, the following assertion for a typical append/3 predicate:

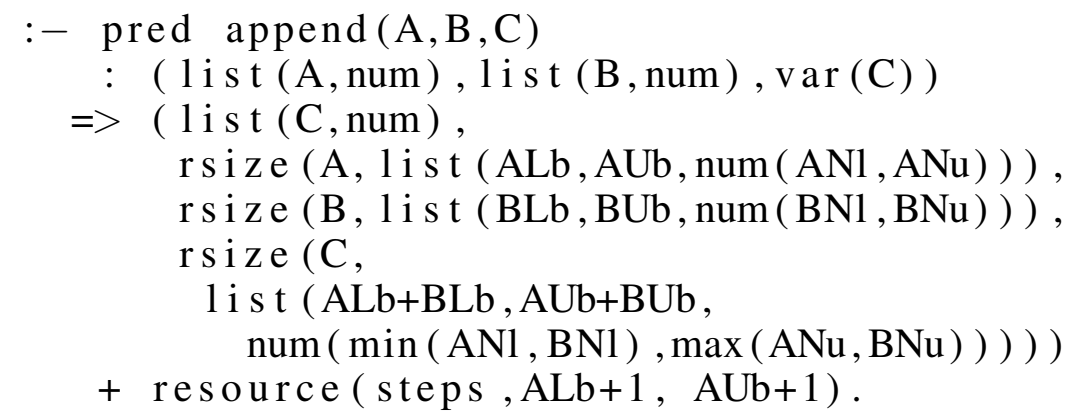

states that for any call to predicate append/3 with the first and second arguments bound to lists of numbers, and the third one unbound, if the call succeeds, then the third argument will also be bound to a list of numbers. It also states that an upper bound on the number of resolution steps required to execute any of such calls is $A U b+1$, a function on the length of list $A$. The rsize terms are the sized types derived from the regular types, containing variables that represent explicitly lower and upper bounds on the size of terms and subterms appearing in arguments.

The global non-functional property resource/3 (appearing in the "+" field), is used for expressing resource usages and follows the schema:

$$
\text { resource(Res_Name, Low_Arith_Expr, Upp_Arith_Expr) }
$$

where Res_Name is a user-provided identifier for the resource the assertion refers to, Low_Arith_Expr and Upp_Arith_Expr are arithmetic functions that map input data sizes to resource usage, representing respectively lower and upper bounds on the resource consumption.

Each assertion can be in a particular status, marked with the following prefixes, placed just before the pred keyword: check (indicating the assertion needs to be checked), checked (it has been checked and proved correct by the system), false (it has been checked and proved incorrect by the system; a compile-time error is reported in this case), trust (it provides information coming from the programmer and needs to be trusted), or true (it is the result of static analysis and thus correct, i.e., safely approximated). The default status (i.e., if no status appears before pred) is check.

\subsubsection{The XC Assertion Language}

The assertions within $\mathrm{XC}$ files use instead a different syntax that is closer to standard $\mathrm{C}$ notation and friendlier for $\mathrm{C}$ developers. These assertions are transparently translated 
into Ciao assertions when $\mathrm{XC}$ files are loaded into the tool. The Ciao assertions output by the analysis are also translated back into $\mathrm{XC}$ assertions and added inline to a copy of the original XC file.

More concretely, the syntax of the XC assertions accepted by our tool is given by the following grammar, where the non-terminal 〈identifier〉 stands for a standard $\mathrm{C}$ identifier,

$\langle$ integer $\rangle$ stands for a standard $\mathrm{C}$ integer, and the non-terminal $\langle$ ground-expr $\rangle$ for a ground expression, i.e., an expression of type $\langle$ expr $\rangle$ that does not contain any $\mathrm{C}$ identifiers that appear in the assertion scope (the non-terminal $\langle$ scope $\rangle$ ).

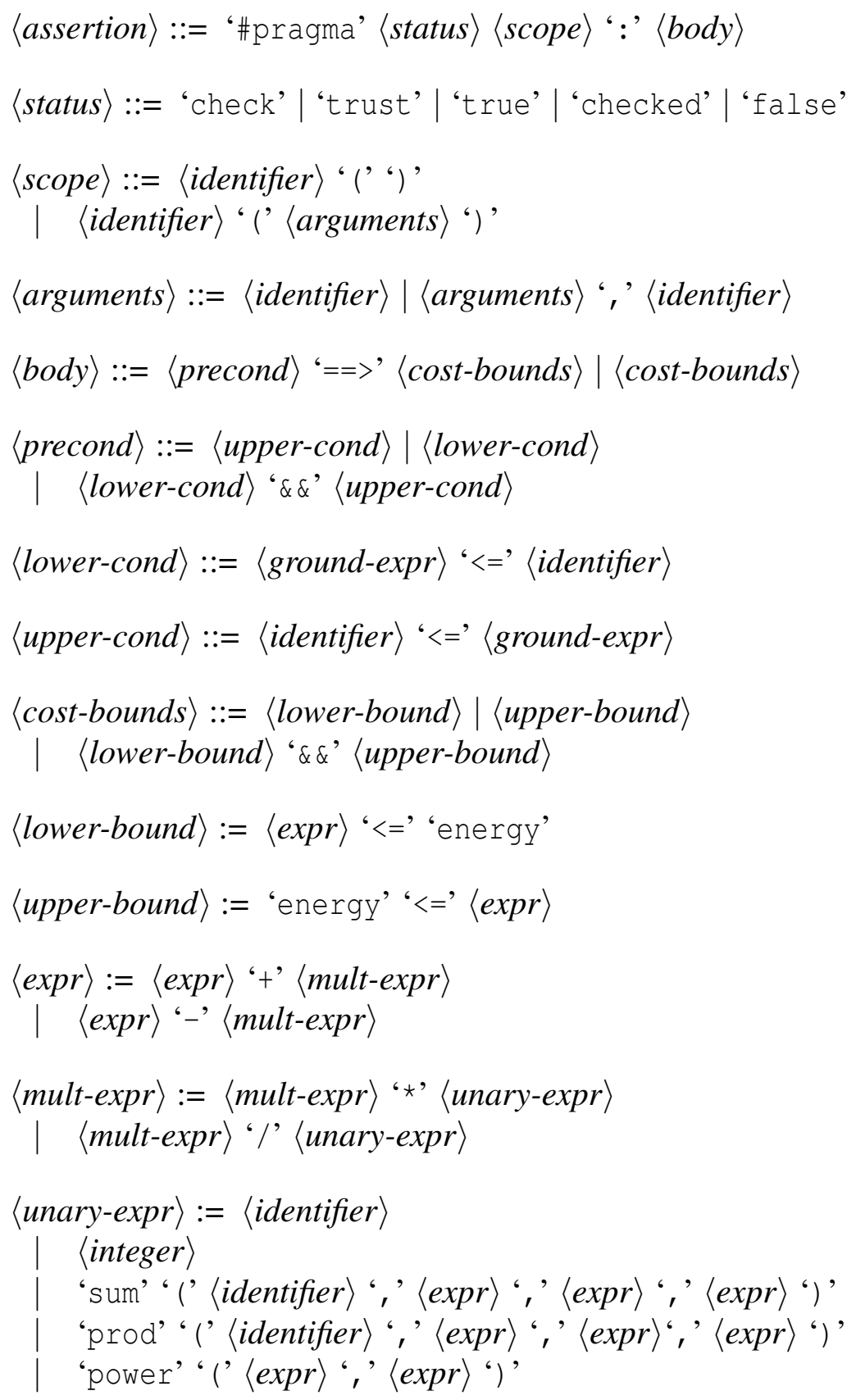




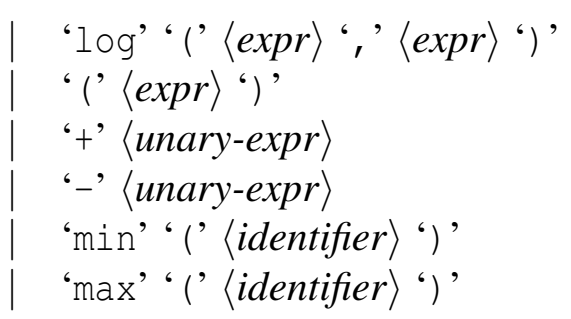

$\mathrm{XC}$ assertions are directives starting with the token \#pragma followed by the assertion status, the assertion scope, and the assertion body. The assertion status can take several values, including check, checked, false, trust or true, with the same meaning as in the Ciao assertions. Again, the default status is check.

The assertion scope identifies the function the assertion is referring to, and provides the local names for the arguments of the function to be used in the body of the assertion. For instance, the scope biquadCascade (state, $x n, N$ ) refers to the function biquadCascade and binds the arguments within the body of the assertion to the respective identifiers state, $\mathrm{xn}, \mathrm{N}$. While the arguments do not need to be named in a consistent way w.r.t. the function definition, it is highly recommended for the sake of clarity. The body of the assertion expresses bounds on the energy consumed by the function and optionally contains preconditions (the left hand side of the $==>$ arrow) that constrain the argument sizes.

Within the body, expressions of type $\langle$ expr $\rangle$ are built from standard integer arithmetic functions (i.e., $+,-,{ }^{*}, /$ ) plus the following extra functions:

- power (base, exp) is the exponentiation of base by exp;

- $\log ($ base, expr) is the logarithm of expr in base base;

- $\operatorname{sum}(i d$, lower, upper, expr) is the summation of the sequence of the values of expr for id ranging from lower to upper;

- prod(id, lower, upper, expr) is the product of the sequence of the values of expr for id ranging from lower to upper;

- $\min (\operatorname{arr})$ is the minimal value of the array arr;

- $\max (\operatorname{arr})$ is the maximal value of the array arr.

Note that the argument of min and max must be an identifier appearing in the assertion scope that corresponds to an array of integers (of arbitrary dimension).

\subsubsection{The General Resource Usage Verification Framework}

In this section we describe the general framework for (static) resource usage verification $[63,64]$ that we have specialized in this paper for verifying energy consumption specifications of XC programs. 
The framework introduced in [63], extends the criteria of correctness as the conformance of a program to a specification expressing non-functional global properties, such as upper and lower bounds on execution time, memory, energy, or user defined resources, given as functions on input data sizes.

Both program verification and debugging compare the actual semantics $\llbracket P \rrbracket$ of a program $P$ with an intended semantics for the same program, which we will denote by $I$. This intended semantics embodies the user's requirements, i.e., it is an expression of the user's expectations. In the framework, both semantics are given in the form of (safe) approximations. The abstract (safe) approximation $\llbracket p \rrbracket_{\alpha}$ of the concrete semantics $\llbracket p \rrbracket$ of the program is actually computed by (abstract interpretation-based) static analyses, and compared directly to the (also approximate) specification, which is safely assumed to be also given as an abstract value $I_{\alpha}$. Such approximated specification is expressed by assertions in the program. Program verification is then performed by comparing $I_{\alpha}$ and $\llbracket p \rrbracket_{\alpha}$.

We assume that the program $P$ is in HC IR form (i.e., a logic program), which is the result of the transformation of the ISA or LLVM IR code corresponding to an XC program. As already said, such transformation preserves the resource consumption semantics, in the sense that the resource usage information inferred by the static analysis (and hence the result of the verification process) is applicable to the original XC program.

\subsubsection{Using the Tool: Example}

As an illustrative example of a scenario where the embedded software developer has to decide values for program parameters that meet an energy budget, we consider the development of an equaliser (XC) program using a biquad filter. In Figure 2.12 we can see what the graphical user interface of our prototype looks like, with the code of this biquad example ready to be verified. The purpose of an equaliser is to take a signal, and to attenuate / amplify different frequency bands. For example, in the case of an audio signal, this can be used to correct for a speaker or microphone frequency response. The energy consumed by such a program directly depends on several parameters, such as the sample rate of the signal, and the number of banks (typically between 3 and 30 for an audio equaliser). A higher number of banks enables the designer to create more precise frequency response curves.

Assume that the developer has to decide how many banks to use in order to meet an energy budget while maximizing the precision of frequency response curves at the same time. In this example, the developer writes an XC program where the number of banks is a variable, say N. Assume also that the energy constraint to be met is that an application of the biquad program should consume less than 125 millijoules (i.e., 125000000 nanojoules). This constraint is expressed by the following check assertion (specification):

\#pragma check biquadCascade (state, $\mathrm{xn}, \mathrm{N}$ ) :

$(1<=\mathrm{N})==>($ energy $<=125000000)$ 


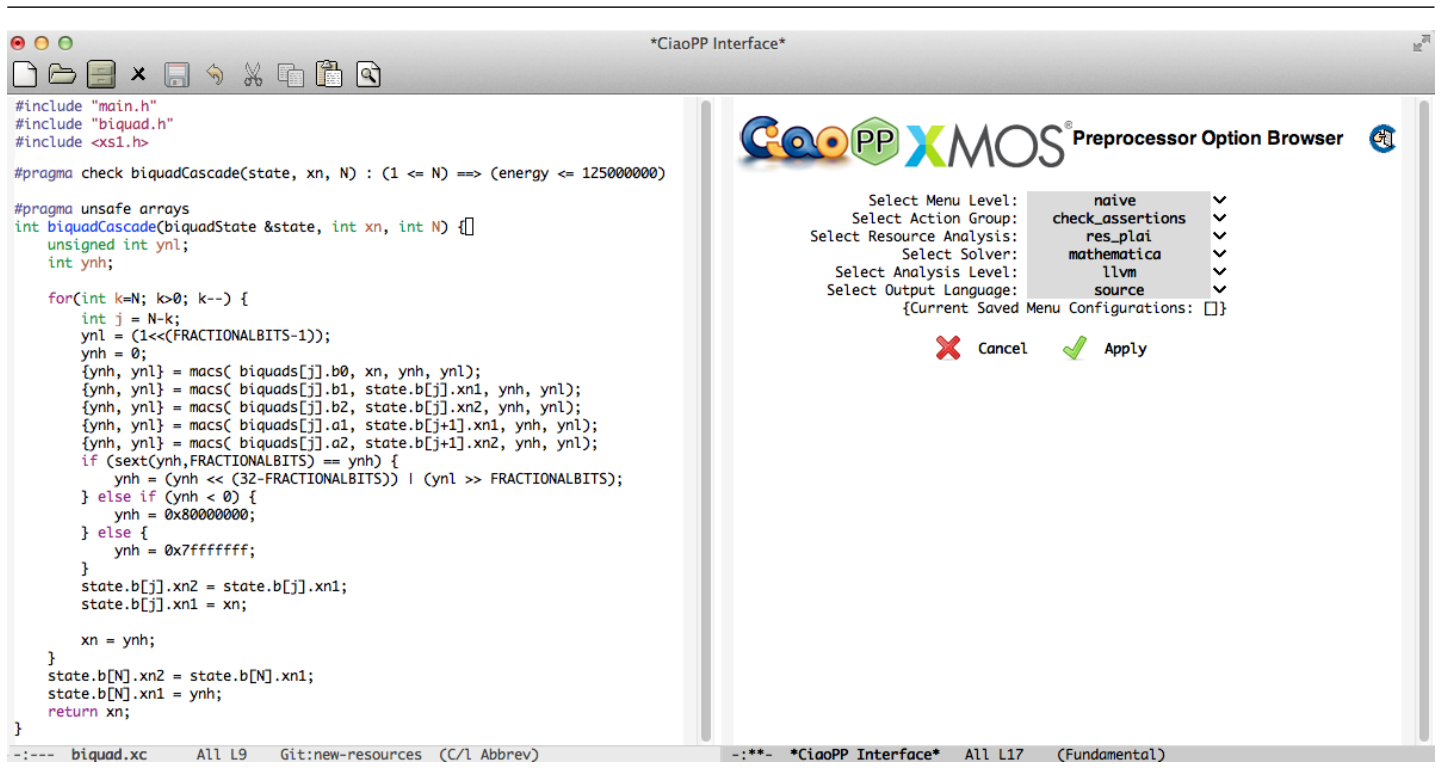

Figure 2.12: Graphical User Interface of the prototype with the XC biquad program.

where the precondition $1<=\mathrm{N}$ in the assertion (left hand side of ==>) expresses that the number of banks should be at least 1 .

Then, the developer makes use of the tool, by selecting the following menu options, as shown in the right hand side of Figure 2.12: check_assertions, for Action Group, res_plai, for Resource Analysis, mathematica, for Solver, llvm, for Analysis Level (which will tell the analysis to take the LLVM IR option by compiling the source code into LLVM IR and transform into HC IR for analysis) and finally source, for Output Language (the language in which the analysis / verification results are shown). After clicking on the Apply button below the menu options, the analysis is performed, which infers a lower and an upper bound function for the consumption of the program. Concretely those bounds are represented by the following assertion, which is included in the output of the tool:

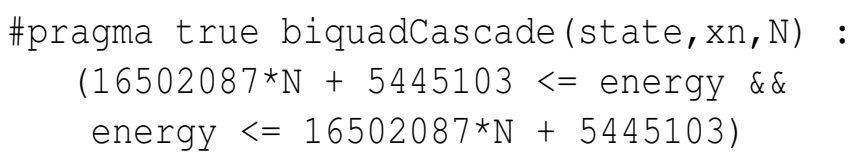

In this particular case, both bounds are identical. In other words, the energy consumed by the program is exactly characterized by the following function, depending on $\mathrm{N}$ only:

$$
E_{\text {biquad }}(\mathrm{N})=16502087 \times \mathrm{N}+5445103 \mathrm{~nJ}
$$

Then, the verification of the specification (check assertion) is performed by comparing the energy bound functions above with the upper bound expressed in the specification, i.e., 125000000, a constant value in this case. As a result, the two following assertions are produced (and included in the output file of the tool): 


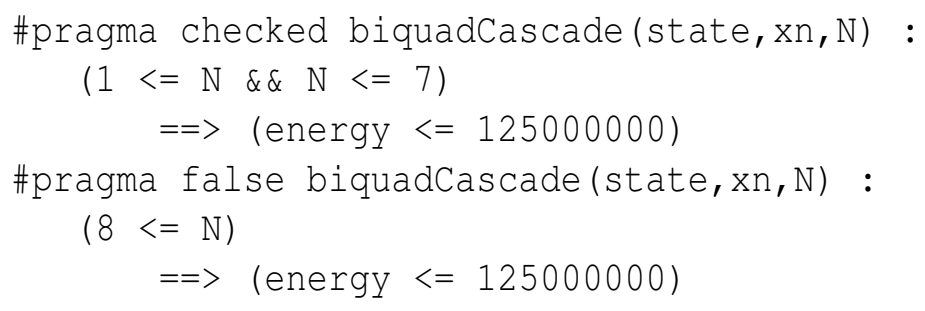

The first one expresses that the original assertion holds subject to a precondition on the parameter $\mathrm{N}$, i.e., in order to meet the energy budget of 125 millijoules, the number of banks $\mathrm{N}$ should be a natural number in the interval $[1,7]$ (precondition $1<=N \& \& \quad N<7$ ). The second one expresses that the original specification is not met (status false) if the number of banks is greater or equal to 8 .

Since the goal is to maximize the precision of frequency response curves and to meet the energy budget at the same time, the number of banks should be set to 7 . The developer could also be interested in meeting an energy budget but this time ensuring a lower bound on the precision of frequency response curves. For example by ensuring that $N \geq 3$, the acceptable values for $N$ would be in the range $[3,7]$.

In the more general case where the energy function inferred by the tool depends on more than one parameter, the determination of the values for such parameters is reduced to a constraint solving problem. The advantage of this approach is that the parameters can be determined analytically at the program development phase, without the need of determining them experimentally by measuring the energy of expensive program runs with different input parameters.

\subsection{Related Work}

Few papers can be found in the literature focusing on static analysis of energy consumption. A similar approach was proposed for upper-bound energy analysis of Java bytecode programs in [75], where the Jimple (a typed three-address code) representation of Java bytecode was transformed into Horn Clauses, and a simple energy model at the Java bytecode level [54] was used. However, this work did not compare the results with actual, measured energy consumption. In this approach and the ones described in this chapter, instantiations for energy consumption of general resource analyzers are used, namely [77] in [75], and [87] in this chapter. Such resource analyzers are based on setting up and solving recurrence equations, an approach proposed by Wegbreit [96] that has been developed significantly in subsequent work [84, 26, 27, 92, 77, 3, 87]. Other approaches to static analysis based on the transformation of the analyzed code into another (intermediate) representation have been proposed for analyzing low-level languages [39] and Java (by means of a transformation into Java bytecode) [4]. In [4], cost relations are inferred directly for these bytecode programs, whereas in [75] the bytecode is first transformed into Horn Clauses. The general resource analyzer in [77] was also instantiated in [68] for the estimation of execution times of logic programs running on a bytecode-based abstract machine. The approach used timing models 
at the bytecode instruction level, for each particular platform, and program-specific mappings to lift such models up to the Horn Clause level, at which the analysis was performed. The timing model was automatically produced in a one-time, programindependent profiling stage by using a set of synthetic calibration programs and setting up a system of linear equations.

By contrast to the generic approach based on CiaoPP, an approach operating directly on the LLVM IR representation is explored in [35]. Though relying on similar analysis techniques, the approach can be integrated more directly in the LLVM toolchain and is in principle applicable to any languages targeting this toolchain. The approach uses the same LLVM IR energy model and mapping technique as the one applied in this chapter.

There exist other approaches to cost analysis such as those using dependent types [44], SMT solvers [6], or size change abstraction [106].

A number of static analyses are also aimed at worst case execution time (WCET), usually for imperative languages in different application domains (see e.g., [97] and its references). The worst-case analysis presented in [47], which is not based on recurrence equation solving, distinguishes instruction-specific (not proportional to time, but to data) from pipeline-specific (roughly proportional to time) energy consumption. However, in contrast to the work presented here and in [68], these worst case analysis methods do not infer cost functions on input data sizes but rather absolute maximum values, and they generally require the manual annotation of loops to express an upperbound on the number of iterations. An alternative approach to WCET was presented in [43]. It is based on the idea of amortisation, which allows to infer more accurate yet safe upper bounds by averaging the worst execution time of operations over time. It was applied to a functional language, but the approach is in principle generally applicable. A timing analysis based on game-theoretic learning was presented in [88]. The approach combines static analysis to find a set of basic paths which are then tested. In principle, such approach could be adapted to infer energy usage. Its main advantage is that this analysis can infer distributions on time, not only average values.

Regarding the support for verification of properties expressed as functions, the closest related work we are aware of presents a method for comparison of cost functions inferred by the COSTA system for Java bytecode [2]. The method proves whether a cost function is smaller than another one for all the values of a given initial set of input data sizes. The result of this comparison is a Boolean value. However, in the approach $[63,64]$ the result is in general a set of subsets (intervals) in which the initial set of input data sizes is partitioned, so that the result of the comparison is different for each subset. Also, [2] differs in that comparison is syntactic, using a method similar to what was already being done in the CiaoPP system: performing a function normalization and then using some syntactic comparison rules. Our technique goes beyond these syntactic comparison rules. Moreover, [2] only covers (generic) cost function comparisons while we have addressed the whole process for the case of energy consumption verification. In a more general context, using abstract interpretation in debugging and/or verification tasks has now become well established. To cite some 
early work, abstractions were used in the context of algorithmic debugging in [57]. Abstract interpretation has been applied by Bourdoncle [14] to debugging of imperative programs and by Comini et al. to the algorithmic debugging of logic programs [20] (making use of partial specifications in [19]), and by P. Cousot [21] to verification, among others. The CiaoPP framework $[16,41,42]$ was pioneering in many aspects, offering an integrated approach combining abstraction-based verification, debugging, and run-time checking with an assertion language.

\subsection{Chapter Conclusions and Future Work}

In this chapter we have introduced a static analysis framework for estimating energy consumption as functions on program input data sizes. The framework allows analyzing programs compiled for the XS1 architecture, into both ISA and LLVM IR representations, based on a Horn clause transformation and uses the ISA level models. For LLVM IR analysis, the approach uses a mapping technique that leverages the existing debugging mechanisms in the XMOS XCore compiler tool chain to propagate an ISAlevel energy model to the LLVM IR level. The two transformations, from ISA and LLVM IR into HC IR, construct a block representation that is supplied, together with the energy values from the energy model, to a parametric resource analyzer that infers the program energy cost as functions on the input data sizes.

Our results suggest that performing the static analysis at the LLVM IR level is a reasonable compromise, since 1) LLVM IR is close enough to the source code level to preserve most of the program information needed by the static analysis, and 2) the LLVM IR is close enough to the ISA level to allow the propagation of the ISA energy model up to the LLVM IR level without significant loss of accuracy for the examples studied. Our experiments are based on single-threaded programs. We also have focused on the study of the energy consumption due to computation, so that we have not tested programs where storage and networking is important. However, this could potentially be done in future work, by using the CiaoPP static analysis, which already infers bounds on data sizes, and combining such information with appropriate energy models of communication and storage. Although the analysis infers sound bound representations in the form of recurrence equations, sometimes the external solvers it uses are not able to find closed form functions for such equations. This is a limitation in applications where such closed forms are needed. Techniques to address such limitation are included in our plans for future work. Our static analysis will also benefit from any improvement of the Computer Algebra Systems used for solving recurrence equations.

It remains to be seen whether the results would carry over to other classes of programs, such as multi-threaded programs and programs where timing is more important. In this sense our results are preliminary, yet they are promising enough to continue research into analysis at LLVM IR level and into ISA-LLVM IR energy mapping techniques to enable the analysis of a wider class of programs, especially multi-threaded programs. 
We have also specialized an existing general framework for resource usage verification for verifying energy consumption specifications of embedded programs. These specifications can include both lower and upper bounds on energy usage, expressed as intervals within which the energy usage is supposed to be included, the bounds (end points of the intervals) being expressed as functions on input data sizes. We have shown through an example, and using the prototype implementation of our approach within the Ciao/CiaoPP system and for the XC language and XS1-L architecture, how our verification system can prove whether such energy usage specifications are met or not, or infer particular conditions under which the specifications hold. These conditions are expressed as intervals of input data sizes such that a given specification can be proved for some intervals but disproved for others. The specifications themselves can also include preconditions expressing intervals for input data sizes. We have illustrated through this example how embedded software developers can use this tool, and in particular for determining values for program parameters that ensure meeting a given energy budget while minimizing the loss in quality of service. 


\section{Evolutionary Modeling of Basic Blocks for Static Analysis of Energy}

\subsection{Introduction}

Different types of resource usage estimations are possible, such as, e.g., probabilistic, average, or safe bounds. However, not all types of estimations are valid or useful for a given application. For example, in order to verify/certify energy budgets, safe upper and lower bounds on energy consumption are required [65, 64]. Unfortunately, current approaches that guarantee that the bounds are always safe tend to compromise their accuracy with overly conservative bounds, which may not be useful in practice. With this safety/accuracy trade-off in mind, we propose an approach that combines a best effort modeling with a safe static analysis, to infer accurate bounds that are on the safe side in most cases, in order to be practical for verification applications, in addition to energy optimization.

Of the small number of static energy analyses proposed to date, only a few ([75] and the ones described in Chapter 2) use resource analysis frameworks that are aimed at inferring safe upper and lower bounds on the resources used by program executions. A crucial component in order for such frameworks to infer information regarding hardware-dependent resources, and, in particular, energy, is a low-level resource usage model, such as, e.g., a model of the energy consumption of individual instructions. Examples of such instruction-level models are [54], at the Java bytecode level, or [51], at the Instruction Set Architecture (ISA) level.

Clearly, the safety of the bounds inferred by analysis depends on the safety of the low-level models. Unfortunately, instruction-level models such as $[54,51]$ provide $a v-$ erage energy consumption values or functions, which are not really suitable for safe upper- or lower-bounds analysis. Furthermore, trying to obtain instruction-level models that provide strict safe energy bounds would result in very conservative bounds. Although when supplied with such models the static analysis would infer high-level energy consumption functions providing strictly safe bounds, these bounds would not be useful in general because of their large inaccuracy. For this reason, the analyses 
in [75] and in Chapter 2 used instead the already mentioned instruction level average energy models $[54,51]$. However, this meant that the energy functions inferred for the whole program were not strict bounds, but rather approximations of the actual bounds, and could possibly be below or above. This trade-off between safety and accuracy is a major challenge in energy analysis. In this chapter we address this challenge by finding a good compromise and providing a best effort technique for the generation of lower-level energy models which are useful and effective in practice for verificationtype applications.

The main source of inaccuracy in current instruction-level energy models is interinstruction dependence (including also data dependence), which is not captured by most models. On the other hand, the concrete sequences of instructions that appear in programs exhibit worst cases that are not as pessimistic as considering the worst case for each of the individual intervening instructions. Based on this, we decided to use branchless blocks of ISA instructions as the modeling unit instead of individual instructions. We divide the (ISA) program into such basic blocks, each a straight-line code sequence with exactly one entry to the block (the first instruction) and one exit from the block (the last instruction). We then measure the energy consumption of these basic blocks, and determine an upper (resp. lower) bound on the energy consumption of each block. In this way the inter-instruction data dependence discussed above and other factors are accounted for within each block. The inter-instruction dependencies between blocks are still modeled in a conservative way, and hence can be one of the sources of inaccuracy. However, such modeling does not affect the safety of the energy bounds. The energy values obtained for each block are supplied to our (safe) static resource analysis, which combines them according to the program control flow and produces functions that give both (practical) upper and lower bounds on the energy consumption of the whole program and its procedures, as functions on input data sizes.

In order to find bounds on the energy consumption of each basic block we use an evolutionary algorithm (EA), varying the basic block's input values and taking energy measurements directly from the hardware for each input combination. This way, we take advantage of the fast search space exploration provided by EAs. The approach in [80] also uses EAs for estimating worst case energy consumption. However, it is applied to whole programs, rather than at the basic block level. A major disadvantage of such an approach is that, if there are data-dependent branches in the programs, as is often the case, the EA quickly loses accuracy, and does not converge since different input combinations can trigger different sets of instructions [80]. This can make the problem intractable. In contrast, our approach combines EAs and static analysis techniques in order to get the best of both worlds. Our approach takes out the treatment of data-dependent branches from the EA, so that the same sequence of instructions is always executed in each basic block. This way, the EA converges and estimates the worst (resp. best) case energy of the basic blocks with higher accuracy. We take care of the program control flow dependencies by using static analysis instead.

For concreteness, in our experiments we focus on the energy analysis of programs 
written in XC [95], running on the XS1-L architecture [66], designed by XMOS. ${ }^{1}$ However, our approach is general enough to be applied as well to the analysis of other architectures and other programming languages and their associated lower-level program representations. $\mathrm{XC}$ is a high-level, C-based programming language that includes extensions for concurrency, communication, input/output operations, and real-time behavior. Our experimental setup infers energy consumption information by processing the ISA (Instruction Set Architecture) code compiled from XC, and reflects it up to the source code level. Such information is provided in the form of functions on input data sizes, and is expressed by means of assertions [40].

The results of our experiments suggest that our best effort approach is quite accurate, in the sense that the inferred energy bounds are close to the actual upper and lower bounds. Furthermore, the energy estimations produced by our approach were always safe, in the sense that they over-approximated the actual bounds (i.e., the inferred upper bounds were above the actual highest energy consumptions and the inferred lower bounds below the actual lowest energy consumptions). We argue thus that our analysis provides a good practical compromise.

In summary, the main contributions of this chapter are:

- A novel approach that combines dynamic and static analysis techniques for inferring more accurate upper and lower bounds on the energy consumption of program executions as functions of input data sizes. The dynamic part is based on EAs, and produces low-level energy models that contain (best effort) upper and lower bounds on the cost of the elementary operations, as opposed to just average values.

- The proposal of a new abstraction level at which to perform the energy modeling of program components, namely at the level of basic (branchless) blocks of ISA instructions, and a method based on EAs to dynamically obtain upper and lower bounds on the energy of such basic blocks with a good safety/accuracy compromise.

- A prototype implementation and experimental study that supports our claims.

In the rest of the chapter, Section 3.2 explains how the information inferred by our approach can be used for the energy consumption verification application. Section 3.3 explains our best effort technique for energy modeling of program basic blocks. Section 3.4 shows how these models are used by the safe static analysis to infer (practical) upper and lower bounds on the energy consumed by programs as functions of their input data sizes. Section 3.5 reports on an experimental evaluation of our approach. Related work is discussed in Section 3.6, and finally Section 3.7 summarizes our conclusions.

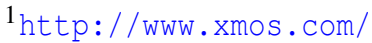




\subsection{Energy Consumption Verification/Certification}

As described in Chapter 2, Section 2.8.2, the lower $\left(E_{l}\right)$ and the upper bound $\left(E_{u}\right)$ inferred by our combined modeling-analysis approach can be used for energy consumption verification and certification. We refer the reader to [63, 64] for a detailed description on how the information inferred by static analysis can be used for general resource usage verification within the CiaoPP system, and to [65] (or Chapter 2, Section 2.8.2) for how it can be specialized for verifying energy consumption specifications. Here we give some intuitive ideas.

Assume that a program specification expresses an energy budget $E_{b}$, e.g., defined by the capacity of the battery; we can conclude the following:

1. $E_{u} \leq E_{b} \Longrightarrow$ that the given program can be safely executed within the existing energy budget.

2. $E_{l} \leq E_{b} \leq E_{u} \Longrightarrow$ it might be possible to complete the execution of the program, but we cannot claim it for certain.

3. $E_{b}<E_{l} \Longrightarrow$ that it is not possible to execute the program (the system will run out of batteries before program execution is completed).

\subsection{Modeling the Energy Consumption of Blocks}

As mentioned before, the first step of our energy bounds analysis is to determine upper and lower bounds on the energy consumption of each basic (branchless) program block. We perform the modeling at this level rather than at the instruction level in order to cater for inter-instruction dependencies. We first identify all the basic blocks of the program, and then perform a best effort profiling of the energy consumption of each basic block for different input data using an EA. These steps are explained in the following sections.

\subsubsection{Identifying the Basic Blocks to be Modeled}

A basic block over an inter-procedural control flow graph (CFG) is a maximal sequence of distinct instructions, $S_{1}$ through $S_{n}$, such that all instructions $S_{k}, 1<k<n$ have exactly one in-edge and one out-edge (excluding call/return edges), $S_{1}$ has one outedge, and $S_{n}$ has one in-edge. A basic block therefore has exactly one entry point at $S_{1}$ and one exit point at $S_{n}$.

In order to divide a program into such basic blocks, the program is first compiled to a lower-level representation, ISA in our case. A dataflow analysis of the ISA representation yields an inter-procedural control flow graph (CFG). A final control flow analysis is carried out to infer basic blocks from the CFG. These basic blocks are further modified so that they can be run and measured independently by the EA. Modifications for each basic block include: 
1. A basic block with $k$ function call instructions is divided into $k+1$ basic blocks without the function call instructions.

2. A number of special ISA instructions (e.g., return, call) are omitted from the block. The cost of such instructions is measured separately and added to the cost of the block.

3. Memory read/write instructions are abstracted to a fixed memory region available to each basic block in order to avoid memory violations.

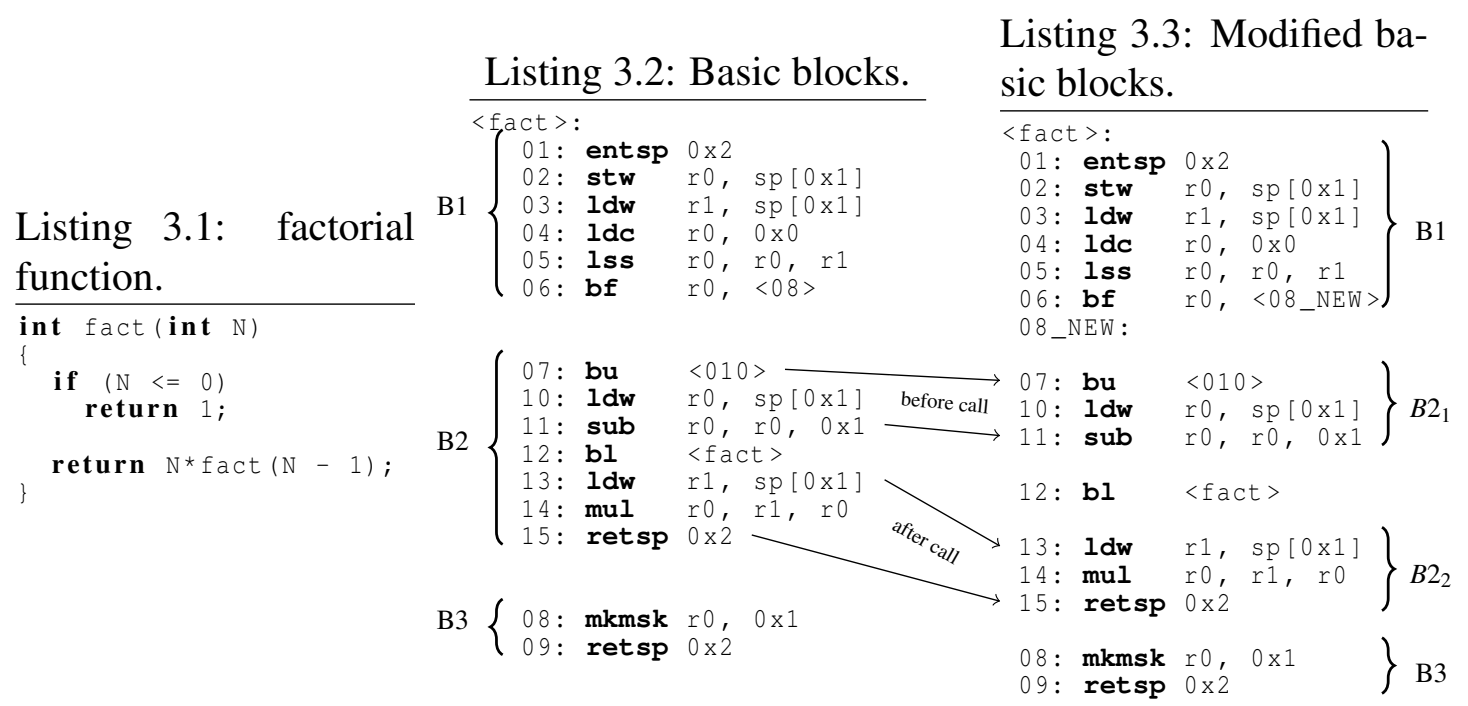

Figure 3.1: Example: Basic block modifications.

An example of the modification 1 above is shown in Figure 3.1, Listing 3.2, which is an ISA representation of a recursive factorial program where the instructions are grouped together into 3 basic blocks B1, B2, and B3. Consider basic block B2. Since it has a (recursive) function call to fact at address 12 , it is divided further into two blocks in Listing 3.3, such that the instructions before and after the function call form two blocks $B 2_{1}$ and $B 2_{2}$, respectively. The energy consumption of these two blocks is maximized (minimized) by providing values to the input arguments to the block (see below) using the EA. The energy consumption of $B 2$ can then be characterized as:

$$
B 2_{e}^{A}=B 2_{1 e}^{A}+B 2_{2 e}^{A}+b l_{e}^{A}
$$

where $B 2_{1 e}^{A}, B 2_{2 e}^{A}$ and $b l_{e}^{A}$ denote the energy consumption of the $B 2_{1}$ and $B 2_{2}$ blocks, and the $b l$ ISA instruction respectively, with approximation $A$ (where $A=$ upper or $A=$ lower).

For each modified basic block, a set of input arguments is inferred. This set is used for an individual representation to drive the EA algorithm to maximize the energy 
consumption of the block. For the entry block, the input arguments are derived from the signature of the function. The set $\operatorname{gen}(B)$ characterizes the set of variables read without being previously defined in block $B$. It is defined as:

$$
\operatorname{gen}(b)=\bigcup_{k=1}^{n}\{v \mid v \in \operatorname{ref}(k) \wedge \forall(j<k) \cdot v \notin \operatorname{def}(j)\}
$$

where $r e f(n)$ and $\operatorname{def}(n)$ denote the variables referred to and defined/updated at a node $n$ in block $b$ respectively.

For the basic blocks in Figure 3.1 in Listing 3.2, the set of input arguments are $\operatorname{gen}(B 1)=\{\mathrm{r} 0\}, \operatorname{gen}\left(B 2_{1}\right)=\{\operatorname{sp}[0 \mathrm{x} 1]\}, \operatorname{gen}\left(B 2_{2}\right)=\{\operatorname{sp}[0 \mathrm{x} 1], \mathrm{r} 0\}$ and $\operatorname{gen}(B 3)=\emptyset$.

\subsubsection{Evolutionary Algorithm for finding Energy Bounds for Basic Blocks}

In this section we detail the most important aspects of the EA used for estimating the maximum (i.e., worst case) and minimum (i.e., best case) energy consumption of a basic block. The only difference between the two algorithms is the way we interpret the objective function: in the first case we want to maximize it, while in the second we want to minimize it.

Individual. The search space dimensions are the different input variables to the blocks. Our goal is to find the combination of input values which maximizes (minimizes) the energy consumption of each block. The set of input variables to a block is inferred using a dataflow analysis (explained in the next section). Thus, an individual is simply an array of input values given in the order of their appearance in the block.

In the initial population, the input values to an individual are randomly assigned to 32-bit numbers. In addition, some corner cases that are known to cause high or low energy consumption for particular instructions are included. ${ }^{2}$

Crossover. The crossover operation is implemented as an even-odd crossover, since it provides more variability than a standard $n$-point crossover. In this crossover the first child is created by taking the first element and every other one after it from one of the parents, e.g., the mother. The second element and every other one come from the other parent, i.e., the father. The second child is created in the opposite way: the first element and every other one after it are taken from the father, while the second and every other one come from the mother. The process is depicted in Figure 3.2, where $P 1$ and $P 2$ are the parents, and $C 1$ and $C 2$ are their children created by the crossover operation.

Mutation. For the purpose of this work we have created a custom mutation operator. Since the energy consumption in digital circuits is mainly the result of bit flipping, we

\footnotetext{
${ }^{2}$ For example all $1 \mathrm{~s}$ for high energy consumption, or all 0 s for low energy consumption as operands to a multiply ISA instruction.
} 


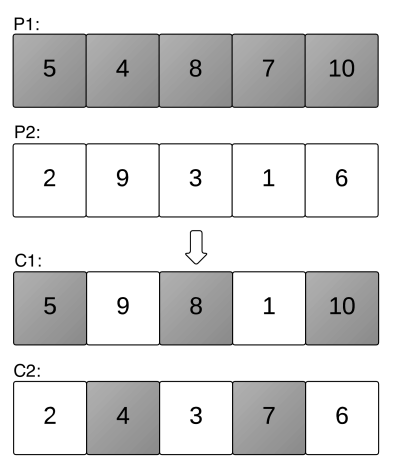

Figure 3.2: Example of even-odd crossover.

believe that the best way to explore the search space is by performing some bit flipping in the mutation operation. This is implemented as follows. For each gene (i.e., input value to the basic block):

1. We create a random 32-bit integer (a random mask).

2. Then we perform the XOR operation of that integer and the corresponding gene. This results in a random flipping of the bits of each gene: only the bits of the gene at positions where the value of the random mask is 1 are flipped.

\begin{tabular}{|c|c|c|}
\hline 32 bits & 32 bits & 32 bits \\
\hline $0 . .0101$ & $0 \ldots 01101$ & $\ldots$ \\
\hline $0 \ldots 0111$ & $0 \ldots 00011$ & $\ldots$ \\
\hline $0 . .0010$ & $0 \ldots 01110$ & $\ldots$ \\
\hline
\end{tabular}

Figure 3.3: Mutation.

The process is depicted in Figure 3.3, where the input values are given as binary numbers.

Objective function. The objective function that we want to maximize/minimize is the energy of a basic block, which is measured directly from the chip. The concrete setting of the experiment will be explained in the following section.

In general, pipeline effects such as stalls (to resolve pipeline hazards), which depend on the state of the processor at the start of the execution of a basic block, can affect the upper/lower bound estimated on the energy consumption of such block. In our approach, intra-block pipeline effects are accounted for, since the dependences among the instructions within a block are captured. However, the inter-block pipeline effects also need to be accounted for. These can be modeled in a conservative way by assuming a maximum stall penalty for the upper bound estimation of each block (e.g., by adding a stall penalty, say three cycles, to the execution time of the block). Similarly, 
for the lower bound estimation a zero stall penalty can be used. To approximate this effect, in [17], the authors characterize each block through pairwise executions with all of its possible predecessors. Each basic block pair is characterized by executing it on an Instruction Set Simulation (ISS) to collect cycle counts.

The XMOS XS1 architecture used in our experiments does not have these pipeline effects by design, since exactly one instruction per thread is executed in a 4-stage pipeline (more details in Section 3.5.1).

\subsection{Energy Consumption of the Program}

Once the (best effort) energy models of each basic block of the program are known, the energy consumption of the whole program is bounded by a (safe) static analyzer (described in Chapter 2) that takes into account the control flow of the program and infers (practical) upper/lower bounds on its energy consumption. We have written the necessary code (i.e., assertions [40]) to feed such analyzer with the block-level upper/lower bound energy model obtained by using the technique explained in Section 3.3.

The analyzer enables a programmer to symbolically bound the energy consumption of a program $P$ on input data $\bar{x}$ without actually running $P(\bar{x})$. It automatically sets up a system of recurrence (cost) equations over the program $P$ that capture its cost (energy consumption) as a function of the sizes of its input arguments $\bar{x}$. Typical size metrics are the actual value of a number, the length of a list or array, etc. [77, 87].

Consider the example in Listing 3.2. The cost equations are set up over the function fact that characterize the energy consumption of the whole function using the approximation $A$ (e.g., upper/lower) of each block inferred by the EA:

$$
\begin{gathered}
\operatorname{fact}_{e}^{A}(R 0)=B 1_{e}^{A}+f a c t \_a u x_{e}^{A}(0 \leq R 0, R 0) \\
f a c t \_a u x_{e}^{A}(B, R 0)= \begin{cases}B 2_{e}^{A}+f a c t_{e}^{A}(R 0-1) & \text { if } B \text { is true } \\
B 3_{e}^{A} & \text { if } B \text { is false }\end{cases}
\end{gathered}
$$

The cost of the fact function is captured by the equation $\operatorname{fact}_{e}^{A}(R 0)$ under an approximation $A$ which in turn depends on $B 1_{e}^{A}$ (i.e., the energy consumption of block $B 1)$ and the equation fact $a u x_{e}^{A}$, which represents the branching originated from the last instruction of block $B 1$. It captures the cost of blocks $B 2$ and $B 3$ based on the condition on the input size $R 0$.

If we assume (for simplicity of exposition) that each basic block has unitary cost in terms of energy consumption, i.e., $B i_{e}=1$ for all $i$, we obtain the energy consumed by fact as a function of its input data size $(R 0)$ : fact $_{e}(R 0)=R 0+1$.

The functions inferred by the static analysis are arithmetic functions (polynomial, exponential, logarithmic, etc.) that depend on input data sizes (natural numbers). The generic resource analyser ensures that the inferred bounds are strict/safe if it is fed with energy models providing safe bounds. 


\subsection{Experimental Evaluation}

In this section we report on an experimental evaluation of our approach to inferring both upper and lower bounds on the energy consumed by program executions, given as functions on input data sizes. The experiments have been performed with XC programs running on the XMOS XS1-L architecture [66]. Such programs include typical embedded applications, e.g., for signal (audio) processing, for which the XS1-L architecture was mainly designed. However, as already said, our approach is general enough to be applied to the analysis of other programming languages (and associated lower level program representations) and architectures as well, provided that energy models for each architecture exist.

\subsubsection{The Platform Modeled}

More concretely, the (development) hardware platform, designed by XMOS, for which we derive the branchless-block-level model, includes a dual-tile board that contains an XS1-A16-128-FB217 processor. The board is fed with a $3.3 \mathrm{~V}$ power supply, allows up to 16 simultaneous tasks on two processors, and supports voltage scaling, although both tiles have to run at the same voltage supply. It also supports frequency scaling, where the tiles can have different frequencies. The XMOS XS1 is a cache-less, predictable architecture by design and manages threads on the hardware. The threads are executed in a round-robin fashion, using a 4-stage pipeline which only permits a single instruction per thread to be active within the pipeline at the same time. This restriction avoids pipeline hazards.

\subsubsection{The Measurement Harness}

In order to take power measurements during execution on real hardware, record and/or display them in real time, the hardware and software harness designed by XMOS, as an extension of the XMOS toolchain, includes:

- A (hardware) debug adapter (xTAG v3.0) that enables power to be measured [99]. The basic principle consists in placing a small shunt resistor of $R_{\text {shunt }}$ ohm (say $0.1 \mathrm{ohm}$ ) in series within the supply line. By measuring the voltage drop on the shunt $V_{\text {shunt }}$, and following Ohm's law, the current is calculated $\left(I_{\text {shunt }}=\right.$ $\left.V_{\text {shunt }} / R_{\text {shunt }}\right)$, which is also the current of the power supply $I_{\text {sup }}=I_{\text {shunt }}$. Then the power consumption is estimated as $V_{\text {sup }} \times I_{\text {sup }}$, where $V_{\text {sup }}$ is the voltage of the power supply. The xTAG v3.0 adapter has an extra connector that carries the analog signals required to estimate the power consumption, as explained above. The measurements regarding these signals are transported to the host computer over USB using the XSCOPE interface [98]. In addition, it includes a protocol designed to enable power measurements and application probing to be performed simultaneously, as well as data to be transported also simultaneously over the USB connection to the host computer. 
- A (software) tool, named xgdb, the debugger that is part of the XMOS toolchain, collects data from the XTAG to be used by the analysis, by connecting to it over a USB interface (using libusb), and reading both ordinary XSCOPE traffic and voltage/current measurements.

\subsubsection{Results and Discussion}

The aim of the experimental evaluation is to perform a first comparison of actual hardware energy measurements against the upper- and lower-bounds on energy consumption obtained by evaluating the functions inferred by our proposed approach (which depend on input data sizes), for each program considered and for different input data sizes.

\begin{tabular}{|c|c|c|c|}
\hline Program & DBr & Upper/Lower Bounds $(\mathbf{n J}) \times 10^{3}$ & vS. HW \\
\hline $\operatorname{fact}(N)$ & $\mathrm{n}$ & $\begin{array}{l}u b=5.1 N+4.2 \\
l b=4.1 N+3.8\end{array}$ & $\begin{array}{r}7 \% \\
-11.7 \%\end{array}$ \\
\hline fibonacci $(N)$ & $\mathrm{n}$ & $\begin{array}{l}u b^{3}=5.2 \operatorname{lucas}(N)+6 \text { fib }(N)-6.6 \\
l b=4.5 \operatorname{lucas}(N)+5 \text { fib }(N)-4.2\end{array}$ & $\begin{array}{r}8.71 \% \\
-4.69 \%\end{array}$ \\
\hline reverse $(A)$ & $\mathrm{n}$ & $\begin{array}{l}u b=3.7 N+13.3(N=\text { length of array } A) \\
l b=3 N+12.5\end{array}$ & $\begin{array}{r}8 \% \\
-8.8 \%\end{array}$ \\
\hline findMax $(A)$ & $\mathrm{y}$ & $\begin{array}{l}u b=5 N+6.9(N=\text { length of array } A) \\
l b=3.3 N+5.6\end{array}$ & $\begin{array}{r}8.7 \% \\
-9.1 \%\end{array}$ \\
\hline selectionSort $(A)$ & $\mathrm{y}$ & $\begin{array}{l}u b=30 N^{2}+41.4 N+10(N=\text { length } \\
\text { of array } A) \\
l b=16.8 N^{2}+28.5 N+8\end{array}$ & $8.7 \%$ \\
\hline $\operatorname{fir}(N)$ & $\mathrm{y}$ & $\begin{array}{l}u b=6 N+26.4 \\
l b=4.8 N+22.9\end{array}$ & $\begin{array}{r}8.9 \% \\
-9.7 \%\end{array}$ \\
\hline $\operatorname{biquad}(N)$ & $\mathrm{y}$ & $\begin{array}{l}u b=29.6 N+10 \\
l b=23.5 N+9\end{array}$ & $\begin{array}{r}9.8 \% \\
-11.9 \%\end{array}$ \\
\hline
\end{tabular}

Table 3.1: Upper and lower bounds accuracy.

The selected benchmarks, which are either iterative or recursive, are shown in Table 3.1. For conciseness, the first column only shows the names of the programs and the arguments that are relevant for their energy-bound functions. The $\mathbf{D B r}$ column expresses whether a benchmark has data-dependent branching or not $(\mathrm{y} / \mathrm{n})$. The third column shows the upper- and lower-bound energy functions (on input data sizes) inferred by our approach, as well as the size metric used. When an input argument (in the first column) is numeric, its size metric is its actual value (and is omitted in the third column). Column vs. HW shows the average deviation of the energy estimations obtained by evaluating such functions, with respect to the actual measurements on the

\footnotetext{
3 The mathematical function lucas $(n)$ satisfies the recurrence relation lucas $(n)=$ lucas $(n-1)+$ $\operatorname{lucas}(n-2)$ with $\operatorname{lucas}(1)=1$ and $\operatorname{lucas}(2)=3$.
} 
hardware. A deviation is positive (resp. negative) if the estimated value is an over(resp. under-) approximation of the actual measurement.

The first two benchmarks are small arithmetic benchmarks. The third benchmark reverse $(A)$ reverses elements of an input array $A$ of size $N$. The list of benchmarks also includes two audio signal processing benchmarks, namely biquad and fir (Finite Impulse Response). Both programs perform a filtering that attenuates or amplifies specific frequency ranges of a given input signal. The fir $(N)$ benchmark computes the inner-product of two vectors: a vector of input samples, and a vector of coefficients. The more number of coefficients $N$, the higher the fidelity, and the more accurately frequencies are modeled, but the more energy is consumed, since it (linearly) depends on $N$, as shown in the third column. The $\operatorname{biquad}(N)$ benchmark is an equalizer that uses a cascade of Biquad filters, where each filter attenuates or amplifies one specific frequency range. The energy consumed depends on the number of banks $N$, typically between 3 and 30 for an audio equalizer. A higher number of banks enables a designer to create more precise frequency response curves. A sorting algorithm (selectionsort) and a simple program for finding the maximum number in an array (findMax) are also included. The latter, which is also part of the former, is a program where datadependent branching can bring significant variations in the worst- and best-case energy consumption.

Figure 3.4(a) depicts the upper- and lower-bound energy functions inferred by the analysis, as well as the actual bounds measured on the hardware for the $\operatorname{fact}(N)$ program (taking different values of $N$ ). In this case, both the actual upper- and lowerbounds coincide, as shown by the middle curve (in red), which depicts the measurements on the hardware. According to Table 3.1, the upper-bound values inferred by the static analysis fed with the model obtained by the EA, over-approximate the actual hardware measurements by $7 \%$, whereas the lower-bound values under-approximate the actual measurements by $11.7 \%$.

Similarly, the findMax benchmark is shown in Figure 3.4(b). Unlike fact, the actual upper- and lower-bound functions of findMax, depending on input arrays of length $N$, do not coincide, due to the data-dependent branching. The actual energy consumption of findMax not only depends on the length of the input array, but also on its contents, and thus cannot be exactly captured by a function that depends on data sizes only (i.e., by abstracting the data by their sizes). A call to findMax with a sorted array in ascending order (of a given length $N$ ) will discover a new max element in each iteration, and hence, update the current max variable, resulting in the actual upper-bound (i.e., worst case of the algorithm). In contrast, if the array is sorted in descending order, the algorithm will find the max element in the first iteration, and the rest of the iterations will never update the current $\max$ variable, resulting in the actual lower-bound (i.e., best case).

Figure 3.4(b) also depicts the upper- and lower-bound energy functions inferred by our approach for findMax, as well as its actual energy bounds measured on the hardware. The former are obtained by evaluating the energy functions in Table 3.2, for different array-lengths $N$, as before. The later are obtained with actual arrays of length 


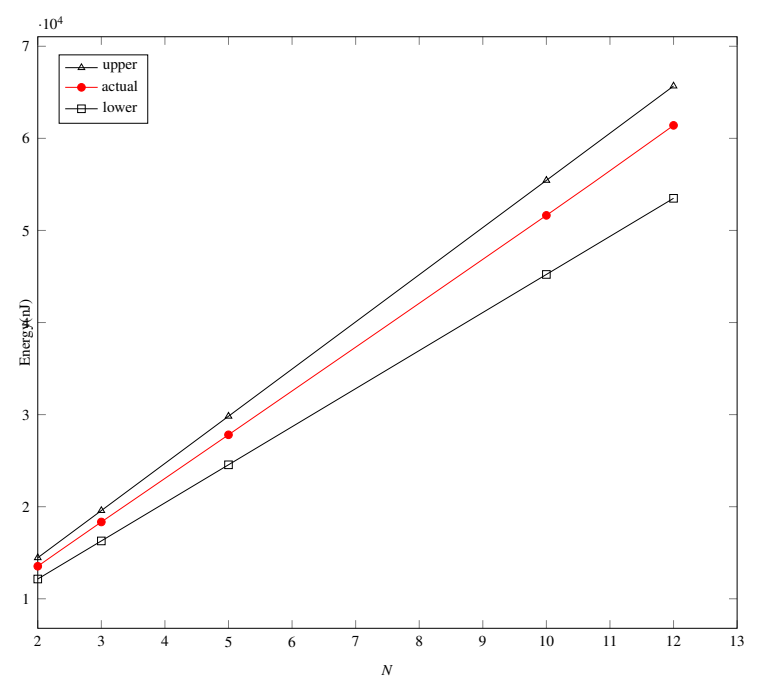

(a) fact.

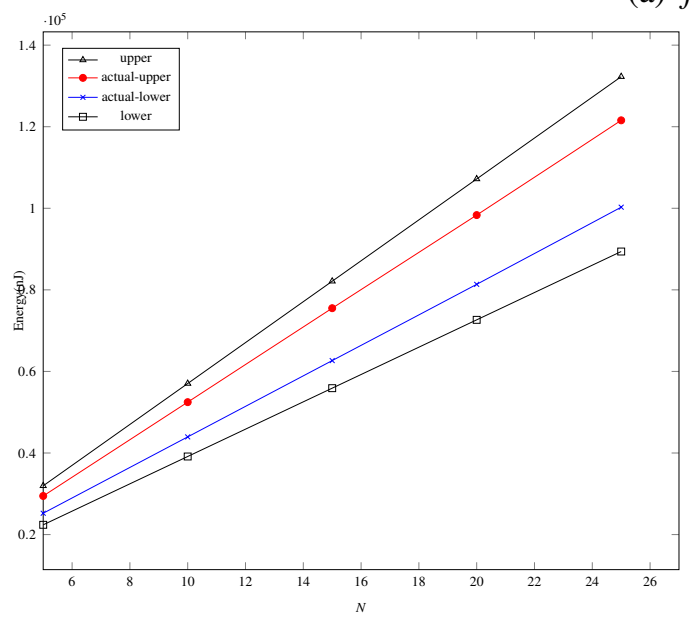

(b) findMax.

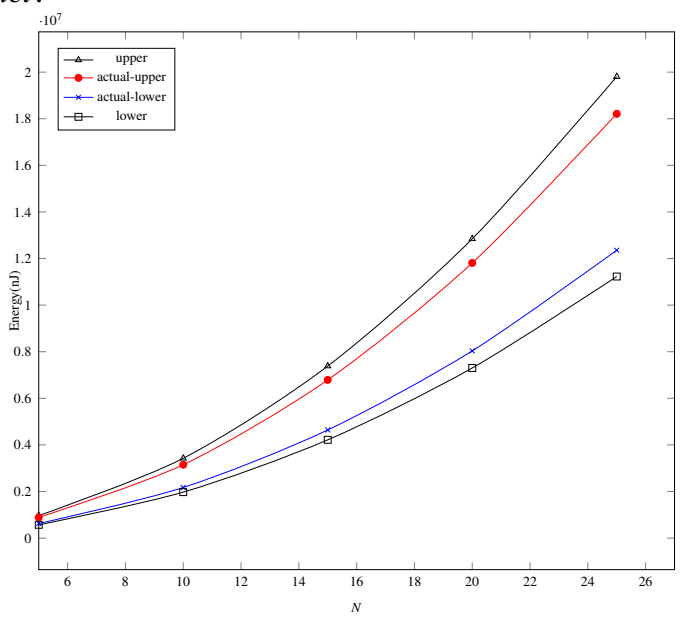

(c) selectionsort.

Figure 3.4: Estimated energy upper/lower bounds vs. actual measurements.

$N$ that give the worst and best cases, as explained above. Note that it is not always trivial to find data that exhibit program worst and best case behaviors. Table 3.1 shows that the inferred upper- (resp. lower-) bounds over- (resp. under-) approximate the actual upper- (resp. lower-) bounds measured on the hardware by $8.7 \%$ (resp. 9.1\%).

The inaccuracies in the energy estimations of our technique come mainly from two sources: the modeling, which assigns an energy value to each basic block as described in Section 3.3, and the static analysis, described in Section 3.4, which estimates the number of times that the basic blocks are executed depending on the input data sizes, and hence, the energy consumption of the whole program. Table 3.2 shows part of the results of our study in order to quantify the inaccuracy originating from those sources. Different executions of the findMax benchmark are shown for different input arrays of length $\mathbf{N}$ (Column $\mathbf{N}$ ). The table is divided in two parts. The first part uses randomly 
generated input arrays of length $\mathbf{N}$, while the second part (three lower rows) uses input arrays that cause the worst- and best-case energy consumption. Column Cost App indicates the type of approximation of the automatically inferred energy functions: upper bound (U) and lower bound (L). Such energy functions are shown in Table 3.1. We have then compared the energy consumption estimations obtained by evaluating the energy function (Column Est) with the observed energy consumption of the hardware measurements (Column Obs). Column D shows the relative harmonic difference between the estimated and the observed energy consumption, given by the formula:

$$
\text { rel_harmonic_diff }(E s t, O b s)=\frac{(E s t-O b s) \times\left(\frac{1}{E s t}+\frac{1}{O b s}\right)}{2}
$$

Column Prof shows the result of estimating the energy consumption using the energy model and assuming that the static analysis was perfect and estimated the exact number of times that the basic blocks were executed. This obviously represents the case in which all loss of accuracy must be attributed to the energy model. The values in Column Prof have been obtained by profiling actual executions of the program with the concrete input arrays, where the profiler has been instrumented to record the number of times each basic block is executed. The energy consumption of the program is then obtained by multiplying such numbers by the values provided by the energy model for each basic block, and adding all of them. Column PrD represents the inaccuracy due to the energy modeling of basic blocks using the EA, which has been quantified as the relative harmonic difference between Prof and the observed energy consumption Obs. The difference between $\mathbf{D}$ and PrD obviously represents the inaccuracy due to the static analysis.

Although the first part of the table, using random data, may give the impression that both the static analysis and the energy modeling contribute to the inaccuracy of the energy estimation of the whole program, the second (lower) part of the table indicates that the inaccuracy only comes from the energy modeling. This is because in the lower part, the comparison was performed with input arrays that make findMax exhibit its actual upper- and lower-bounds (depending on the length of the array). In this case, Columns Est and Prof show the same values, which means that there was no inaccuracy due to the static analysis (regarding the inference of the actual upper-and lower-bound functions), and that the overall inaccuracy is due to the over- and underapproximation in the EA to model energy consumption of each basic block.

Table 3.3 shows a similar experiment for the reverse program, which has no datadependent branching. Since the number of operations performed by reverse is actually a function of the length of its input array (not of its contents), Columns Est and Prof show the same values for random data (unlike for findMax), which means that no inaccuracy comes from the static analysis part.

Regarding the time taken by the EA, it can vary depending on the parameters it is initialized with, as well as the initial population. This population is different every time the EA is initiated, except for a fixed number of individuals that represent corner cases. In the experiments, the EA is run for up to a maximum of 20 generations, and 


\begin{tabular}{|c|c|c|c|c|c|c|}
\hline \multirow{2}{*}{$\mathbf{N}$} & \multirow{2}{*}{$\begin{array}{c}\text { Cost } \\
\text { Appx }\end{array}$} & \multicolumn{3}{|c|}{$\operatorname{Energy}(\mathbf{n J}) \times 10^{3}$} & \multirow{2}{*}{ D \% } & \multirow{2}{*}{$\operatorname{PrD} \%$} \\
\hline & & Est & Prof & Obs & & \\
\hline \multicolumn{7}{|c|}{ Random array data } \\
\hline \multirow{2}{*}{5} & $\mathrm{~L}$ & 22.3 & 24.9 & \multirow{2}{*}{27.3} & -20.1 & -9.2 \\
\hline & $\mathrm{U}$ & 31.9 & 30.2 & & 15.6 & 10 \\
\hline \multirow{2}{*}{15} & $\mathrm{~L}$ & 55.9 & 61.8 & \multirow{2}{*}{69.1} & -17 & -11 \\
\hline & $\mathrm{U}$ & 82.1 & 75.1 & & 21 & 8.3 \\
\hline \multirow{2}{*}{25} & $\mathrm{~L}$ & 89.4 & 99.6 & \multirow{2}{*}{110.9} & -17.6 & -10.7 \\
\hline & $\mathrm{U}$ & 132.2 & 120.8 & & 21.7 & 8.5 \\
\hline \multicolumn{7}{|c|}{ Actual worst- and best-case array data } \\
\hline \multirow{2}{*}{5} & $\mathrm{~L}$ & 22.3 & 22.3 & 25.2 & -12.2 & -12.2 \\
\hline & $\mathrm{U}$ & 31.9 & 31.9 & 29.4 & 8.1 & 8.1 \\
\hline \multirow{2}{*}{15} & $\mathrm{~L}$ & 55.9 & 55.9 & 62.6 & -11.3 & -11.3 \\
\hline & $\mathrm{U}$ & 82.1 & 82.1 & 75.5 & 8.3 & 8.3 \\
\hline \multirow{2}{*}{25} & $\mathrm{~L}$ & 89.4 & 89.4 & 100.2 & -11.4 & -11.4 \\
\hline & $\mathrm{U}$ & 132.2 & 132.2 & 121.5 & 8.4 & 8.4 \\
\hline
\end{tabular}

Table 3.2: Source of inaccuracies in findMax prediction: analysis vs. modeling.

is stopped when the fitness value does not improve for four consecutive generations. In all the experiments the biquad benchmark took the most time (a maximum time of 230 minutes) for maximizing the energy consumption. In contrast, the fact benchmark took the least time (a maximum time of 121 minutes). The times remained within the 150-200 minutes range on average. Time speed-ups were also achieved by reusing the EA results for sequences of instructions that were already processed in a previous benchmark (e.g., return blocks, loop header blocks, etc.). This makes us believe that our approach could be used in practice in an iterative development process, where the developer gets feedback from our tool and modifies the program in order to reduce its energy consumption. The first time the EA is run would take the highest time, since it would have to determine the energy consumption of all the program blocks. After a focused modification of the program that only affects a small number of blocks, most of the results from the previous run could be reused, so that the EA would run much faster during this development process. In other words, the EA processing can easily be made incremental.

The static analysis, on the other hand, is quite efficient, with analysis times of about 4 to 5 seconds on average, despite the naive implementation of the interface with external recurrence equation solvers, which can be improved significantly.

\subsection{Related Work}

Static analysis of the energy consumed by program executions has received relatively little attention until recently. An analysis of Java bytecode programs that inferred 
Chapter 3. Evolutionary Modeling of Basic Blocks for Static Analysis of Energy

\begin{tabular}{|c|c|c|c|c|c|c|}
\hline \multirow{2}{*}{$\mathbf{N}$} & \multirow{2}{*}{$\begin{array}{c}\text { Cost } \\
\text { Appx }\end{array}$} & \multicolumn{3}{|c|}{$\operatorname{Energy}(\mathbf{n J}) \times 10^{3}$} & \multirow{2}{*}{ D \% } & \multirow{2}{*}{ PrD \% } \\
\hline & & Est & Prof & Obs & & \\
\hline \multicolumn{7}{|c|}{ Random array data } \\
\hline \multirow{2}{*}{5} & $\mathrm{~L}$ & 28 & 28 & \multirow{2}{*}{29} & -3.5 & -3.5 \\
\hline & $\mathrm{U}$ & 31.8 & 31.8 & & 9.2 & 9.2 \\
\hline \multirow{2}{*}{15} & $\mathrm{~L}$ & 59 & 59 & \multirow{2}{*}{64} & -8.1 & -8.1 \\
\hline & $\mathrm{U}$ & 68.8 & 68.8 & & 7.2 & 7.2 \\
\hline \multirow{2}{*}{25} & $\mathrm{~L}$ & 90 & 90 & \multirow{2}{*}{98} & -8.5 & -8.5 \\
\hline & $\mathrm{U}$ & 105.8 & 105.8 & & 7.7 & 7.7 \\
\hline
\end{tabular}

Table 3.3: Source of inaccuracies in reverse prediction: analysis vs. modeling.

upper-bounds on energy consumption as functions on input data sizes was proposed in [75], where the Jimple (a typed three-address code) representation of Java bytecode was transformed into Horn Clauses, and a simple energy model at the Java bytecode level [54] was used. However the energy model used average estimations of the Java opcodes, which are not suitable for verification applications. Furthermore, this work did not compare the results with actual, measured energy consumption. As already commented, a similar approach is proposed in Chapter 2 (or $[62,61])$ for the analysis of XC programs. However, it used an ISA-level model that provided average energy values too, which implied the same problem in verification. Other approaches to static analysis based on the transformation of the analyzed code into another (intermediate) representation have been proposed for analyzing low-level languages [39] and Java (by means of a transformation into Java bytecode) [4]. In [4], cost relations are inferred directly for these bytecode programs, whereas in [75] the bytecode is first transformed into Horn Clauses.

Other work has taken as its starting point techniques referred to generally as WCET (Worst Case Execution Time Analyses), which have been applied, usually for imperative languages, in different application domains (see e.g., [97] and its references). These techniques generally require the programmer to bound the number of iterations of loops, and then apply an Implicit Path Enumeration technique to identify the path of maximal consumption in the control flow graph of the resulting loop-less program. This approach has inspired some worst case energy analyses, such as the one presented in [48]. It distinguishes instruction-specific (not proportional to time, but to data) from pipeline-specific (roughly proportional to time) energy consumption. The approach also takes into account complex issues such as branch prediction and cache misses. However, they rely on the user to identify the input which will trigger the maximal energy consumption. In [94] the same approach is applied and further refined for estimating hard (i.e., over-approximated) energy bounds. The main novelty of this work consists in introducing relative energy models (implemented at the LLVM level in this case), where the energy of each instruction is given in relation to each other (e.g., if we assume that all the instructions have relative energy 1, this means that they all have the same absolute energy), which does not depend on the specific hardware, but can be 
applied for all the platforms where a mapping between LLVM and low-level assembly instructions exists. On the other hand, in the situations when the energy bounds are not hard (i.e., the application allows their violation) they use a genetic algorithm to obtain an under-approximation of the energy bounds. However, this approach loses accuracy when there are data-dependent branches present in the program, since different inputs can lead to the execution of different set of instructions. A similar approach is used in [80] to find the worst-case energy consumption of two benchmarks using a genetic algorithm. In contrast to our approach, the evolutionary algorithm is applied to whole programs, and these do not have any data-dependent branching. The authors further introduce probability distributions for the transition costs among pairs of independent instructions, which can be then be convolved to give a probability distribution of the energy for a sequence of instructions.

In contrast to the work presented here and in [68], all these WCET-style methods (either for execution time or energy) do not infer cost functions on input data sizes but rather absolute maximum values, and, as mentioned before, they generally require the manual annotation of all loops to express an upper bound on the number of iterations, which can be tedious (or impossible) and effectively reduces the case to that of programs with no loops.

Another alternative approach to WCET-style methods was presented in [43]. It is based on the idea of amortization, which allows inferring more accurate yet safe upper bounds by averaging the worst execution time of operations over time. It was applied to a functional language, but the approach is in principle generally applicable. In principle, this approach could be adapted to infer energy usage.

\subsection{Chapter Conclusions and Future Work}

We have proposed a combined static/dynamic approach for estimating parametric upper and lower bounds on the energy consumption of a program. The dynamic part, based on an EA, is a best effort approach to approximating the maximum/minimum energy consumption of the basic blocks in the program. Such blocks contain multiple instructions, which allows this phase to capture inter-instruction dependencies. Moreover, the basic blocks are branchless, which makes the EA approach quite practical and efficient, and the energy values inferred by it are accurate, since no control flow-related variations occur. A safe static analysis is then used to combine the energy values obtained for the blocks according to the program control flow, and estimate energy consumption bounds of the whole program that depend on input data sizes. In the experiments we performed on a set of benchmarks, the upper and lower bounds obtained were always safe and quite accurate. Such results suggest that our approach offers a good safety/accuracy compromise, estimating bounds that are practical for its application to energy verification and optimization.

Future work includes improving the EA by introducing data analysis of the input values to each block in order to capture the inter-block data dependencies. This will also speedup the EA since it will have bounds on the input values that it can explore. 


\section{Transformational Approach to Parametric Accumulated-Cost Static Profiling}

\subsection{Introduction and Motivation}

The execution of software consumes resources such as time, energy, and memory. The goal of automatic program resource analysis is to infer the resources that a program uses as a function on the size of the input data or other environmental parameters of the program, without actually executing the program. Previous work on this topic, mainly for inferring asymptotic time complexity bounds, goes back to the 1970s. Recent research has adapted and extended these techniques for inferring other resources, including for example energy (see Chapter 2 or $[62,61]$ ).

In this chapter we investigate an extension of this problem which, although based on the same essential techniques, has a different range of applications. Rather than estimating the total resource usage of a program, we wish to perform static profiling of its resource usage. This means that we intend to discover, for selected parts of the program, an estimate of the resources used by those parts. As before, the estimates will be parameterised by input sizes. However, these input sizes will be of the entry procedure/function, unlike the input sizes of the selected parts, as in the standard resource analysis.

There are several motivations for this research. Firstly, a profile of the resource usage of the program can show the developer which parts of the program are the most resource critical. For example, it can expose the cost of functions that are perhaps not particularly resource hungry by themselves but which are called many times. Such parts are natural targets for optimization, since there a small improvement can yield important savings. Secondly, there are cases where the overall resource functions of a program might not be obtainable. This can be for instance because some program parts are too complex for analysis or because the code for some parts is not available and the cost cannot even be reasonably estimated. In this case useful information may still be obtained by excluding such parts from the analysis, obtaining information about the resource usage for the rest of the program. Thirdly, resource usage models (for ex- 
ample Tiwari's energy consumption model [90]) are sometimes based on summing the individual resource usage of basic components of the program. The analysis presented here fits naturally with such models. Finally, in cases where a program has mutually recursive functions/predicates, the standard cost analysis infers similar resource functions for each recursive function. In such cases, a static profile finds precisely the resource functions for each mutually recursive part of the program, and helps identify the parts that are responsible for most of the cost.

The traditional profiling techniques are dynamic (i.e., require executing the program on some particular input) and are based either on code instrumentation, i.e., introducing additional pieces of code in the sections to be measured, or on running a process that performs the profiling together with the measured program. In both cases, the dynamic profiler introduces an overhead in the resource measured that needs to be properly discriminated, which is non trivial. For example, it may be the case that an instruction in the original program has a very different energy consumption in the presence of code added by the profiler just before it. In contrast, the static profiling approach we propose in this chapter obtains safe upper and lower bounds on resource consumption, because it is based on the semantics of the program rather than particular executions of it. I.e., the results are valid for all possible program inputs.

Our starting point is the well-developed technique of extracting recurrence relations that express resource usage functions [96, 84, 26, 25, 27, 3]. These are then solved to get a closed-form function expressing the (bounds on) parameterised resource usage. In our work we will make use the CiaoPP program analysis framework, which includes a set of generic resource analyses based on these techniques. In particular, we will use the analysis described in [87]. CiaoPP operates on an intermediate semantic program representation based on Horn Clauses [67], that we will refer to as the "HC IR." By transforming the input language into this intermediate representation, the CiaoPP framework has been shown capable of analyzing imperative programs at the source, bytecode, or binary level with competitive precision and efficiency (see [67, 76, 75] and Chapter 2 for details).

Our approach to static profiling is based on a transformation that is performed at the level of the CiaoPP Horn Clause-based intermediate representation. The proposed transformation allows a standard cost analyzer (CiaoPP in our experiments) to statically infer functions that return bounds on accumulated costs depending on input data sizes, for a number of predefined program points of interest (predicates in our case), referred to as cost centers. Intuitively, given a program $\mathcal{P}$, the cost accumulated in a given predicate $\mathrm{p} \in \mathcal{P}$ is defined in the context of the execution of a single call to another predicate $q \in \mathcal{P}$. It expresses the addition of (part of) the resource usages corresponding to the execution of all calls to predicate $\mathrm{p}$ generated by a single call to predicate $q \in \mathcal{P}$.

In the rest of the chapter, Section 4.2 presents informally a general model of (dynamic) profiling and how we turn it into a static version. Section 4.3 reviews established techniques for cost analysis based on extracting and solving cost relations. Section 4.4 formalizes our notion of accumulated cost. Section 4.5 describes the im- 
plementation of the technique, based on a source-to-source transformation. Section 4.6 reports some experimental results. In Section 4.7 we comment on some related work and finally Section 4.8 concludes, discussing future directions.

\subsection{From Dynamic Profiling to Static Profiling}

We start by presenting informally a general model of (dynamic) profiling and how we turn it into a static version. Our model is based on the notion of cost centers, inspired from the work of Sansom and Peyton Jones [85] and Morgan and Jarvis [73]. This approach was also applied to Logic Programs and extended to perform run-time checking of non-functional properties in [69]. Intuitively a cost center provides a dynamic scoping mechanism to uniquely attribute the execution costs of a part of the code to an identifier. The scope of the cost center is dynamic in the sense that execution costs of code that are not explicitly associated to a cost center are dynamically attributed to the same cost center as the caller. For a number of languages it is convenient to identify the cost centers with (a subset of) functions, procedures, or predicates. In this chapter we follow this path. Alternatively, cost centers can be defined by special scoping constructs [85].

As an example, ${ }^{1}$ assume that a programmer wishes to profile a program which uses the following variance () function (variance () naively computes the variance of an array of integers):

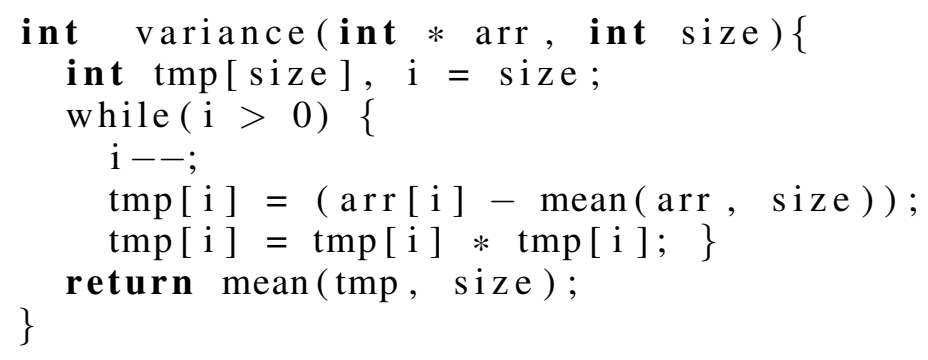

Assume that mean() is a given function that computes the mean of an integer array. First consider that both mean() and variance () are cost centers. In this case the actual execution costs of the code that appears textually within the variance () function will be aggregated at each call to such function and will be attributed to the variance () cost center. However the cost of calls to mean() -including those made from variance () - will not be attributed to variance (). Now consider the case where variance () is declared a cost center, but mean() is not. In this case the execution costs of calls to mean() made from the variance () function will be also aggregated to those of variance () (but not those made from other points in the program).

Returning to the case where both variance () and mean() are declared as cost centers, assume that the programmer profiles the energy consumption (measured as nano

\footnotetext{
${ }^{1}$ As mentioned in the introduction, CiaoPP's analyses deal with programs written in such C-like languages (among others) by analyzing corresponding Horn Clause representations.
} 
joules, $n J)$ of a call to the variance () function over the array $\{1,2,3,4\}$, on some particular architecture. Assume that the result of the profiler is that 74.7 units of energy are accumulated in the variance () cost center and 464.4 units in the mean() cost center. Since mean() is called 4 times, the cost of a single call to it (with the array above) would be $116.1 \mathrm{~nJ}(464.4 / 4)$. If only variance () were declared a cost center, the profiler would have accumulated all the cost in it, i.e., $464.4+74.7 \mathrm{~nJ}$. In such a case, the cost measured by the profiler would be the same as what we call the standard cost of a (single) call to variance () with the given array (i.e., $539 n J$ ).

Since the accumulated value in the mean() cost center is much larger than that accumulated in the variance () cost center, this indicates that for this particular call most of the energy is consumed inside the mean() function, i.e., that this function is responsible for most of the standard cost of the call to variance (). This can be a strong indicator that it may be worthwhile to either optimize the body of mean() or try to reduce the number of times it is called. Note, however, that with just this data, which come from a run with a particular input, the programmer does not really have any guarantees that the results are representative of the general behavior of the program for all inputs. This problem is usually tackled by repeating the process on a large set of different inputs. This can lead to more indicative results, but still has several drawbacks. First, this process can be very long, because profiling usually introduces additional execution costs, which get multiplied by the number of inputs. Second, and more importantly, even if a large number of inputs is used, this still does not provide a strong guarantee, i.e., there may be some corner case inputs for which the call behaves in a very different way. Finally, the approach does not allow the comparison of the asymptotic cost accumulated in the different cost centers.

To overcome the problems outlined above, we propose to statically infer (lower and upper) bounds on the cost accumulated in the cost centers as functions of the sizes of the input data to the profiled call (the call variance () in our example). In the example above, the system we have implemented infers (for the resource "energy" 2 ) that for a call to variance () with a list of size size, the costs accumulated in the variance and mean() functions are $24.32+$ size $\times 12.59$ and $23.03+17.46 \times$ size $^{2}+40.49 \times$ size energy units (nano joules) respectively. In this case the system infers these expressions for both the upper and lower bounds, which means that they are exact costs. Hence, the programmer does have the guarantee that for all non-trivial calls (i.e., for all calls with non-empty lists) and for any input data, the code of mean() consumes most of the energy. In this case an obvious improvement can be made, since the call to mean(arr, size ) can be safely moved outside the loop:

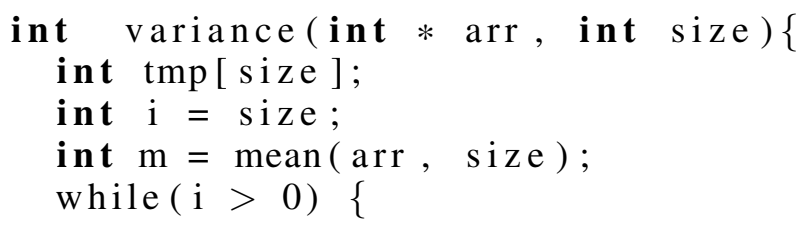

\footnotetext{
${ }^{2}$ Using as back-end analysis the energy analysis of Chapter 2 on an XCore XS1 processor with the program compiled by the XMOS xcc compiler without optimization.
} 
Chapter 4. Transformational Approach to Parametric Accumulated-Cost Static Profiling

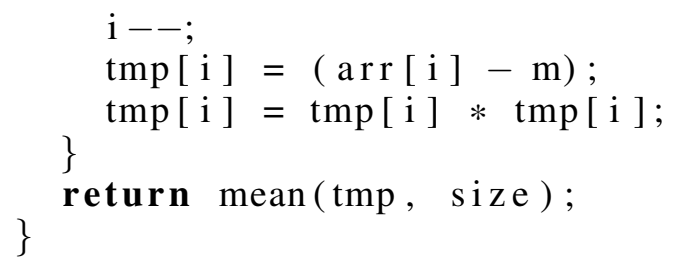

For this version of the program, the system infers that the costs accumulated in the variance () and mean() functions are $28.18+$ size $\times 8.73$ and $46.06+34.92 \times$ size energy units (nano joules) respectively. For brevity and simplicity we chose a program that is rather naive and where the optimization is obvious (and would in fact be done by some compilers automatically), but the same reasoning applies to more complex cases that are not easy to spot without profiling information. Furthermore, the static profiling functions can also be used for guiding automatic optimization by the compiler.

\subsection{The Classical Cost Relations-based Parametric Static Analysis}

The approach to cost analysis based on setting up and solving recurrence equations was proposed in [96] and has been developed significantly in subsequent work. For example, in [84] an automatic upper-bound analysis was presented based on an abstract interpretation of a step-counting version of a functional program, in order to infer both execution time and execution steps. However, size measures could not automatically be inferred and the experimental section showed few details about the practicality of the analysis. In the context of Logic Programming, a semi-automatic analysis was presented in $[26,25]$ that inferred upper-bounds on the number of execution steps, given as functions on the input data sizes. This work also proposed techniques to address the additional challenges posed by the Logic Programming paradigm, as, for example, dealing with the generation of multiple solutions via backtracking. However, a shortcoming of the approach was its loss in precision in the presence of divide-andconquer programs in which the sizes of the output arguments of the "divide" predicates are dependent. This approach was later fully automated (by integrating it into the CiaoPP system and automatically providing modes and size measures) and extended to inferring both upper- and lower-bounds on the number of execution steps (which is non-trivial because of the possibility of failure) in [27, 42]. In addition, [27] introduced the setting up of non-deterministic recurrence relations for the class of divide-and-conquer programs mentioned above, and proposed a technique for computing approximated closed form bound functions for some of them. Such a technique was based on bounding the number of terminal and non-terminal nodes in the set of computation trees corresponding to the evaluation of the non-deterministic recurrence relations, and bounding the cost of such nodes. Non-deterministic recurrence relations were also used and further developed in [3] (named Cost Relations). The approach in $[26,25,27]$ was generalized in [77] to infer user-defined resources (by using an extension of the Ciao assertion language [40]), and was further improved in [87] by 
defining the resource analysis itself as an abstract domain that is integrated into the PLAI abstract interpretation framework [74, 83] of CiaoPP, obtaining features such as multivariance, efficient fixpoints, and assertion-based verification and user interaction. A significant additional improvement brought about by [87] is that it is combined with a sized types abstract domain, which allows the inference of non-trivial cost bounds when they depend on the sizes of input terms and their subterms at any position and depth. Recently, many other approaches have been proposed for resource analysis $[92,44,36,46,79,33,3,5]$. While based on different techniques, all these analyses infer, for all predicates $\mathrm{p}$ of a given program $\mathcal{P}$, an approximation of the notion of cost that we call the standard cost or single call cost. Most of them infer an upper bound, while others infer both upper and lower bounds. The following example shows this (for the case of CiaoPP) and also illustrates that this concept of cost may not be directly useful for locating performance bottlenecks.

Consider the following implementation of an eval $(E, M, R)$ predicate that evaluates modulo $2^{\mathrm{M}}$ a given expression $\mathrm{E}$ built from additions and multiplications. This implementation assumes that two predicates are given: add $(A, B, M, R)$ and mult $(A, B, M, R)$, that respectively add and multiply two infinite precision numbers $A$ and $B$ modulo $2^{\mathrm{M}}$, and unify the result with $R$.

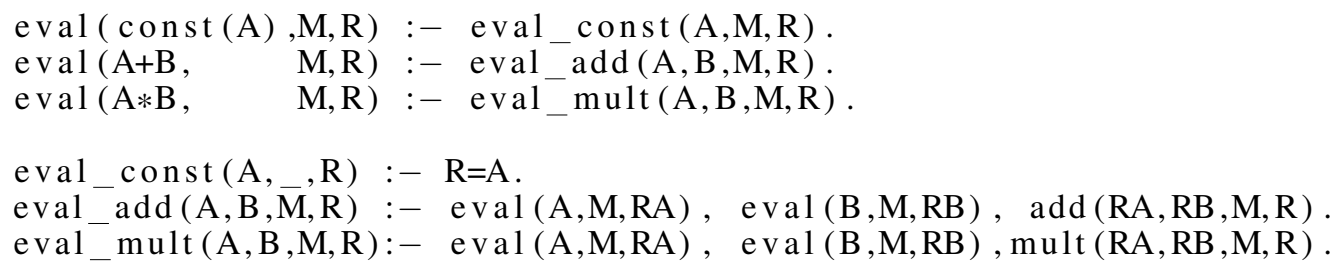

For the sake of simplicity, assume that all the costs are null except those related to the evaluation of add/4 and mult/4. Assume that the cost of the evaluation of $\operatorname{add}(A, B, M, R)$ is $M$ and the cost of the evaluation of mult $(A, B, M, R)$ is $M^{2}$. Under these assumptions, the standard CiaoPP cost analysis infers that the cost of the evaluation of eval $(E, M, R)$ is bounded by $\left(2^{\operatorname{depth}(E)}-1\right) \times\left(M+M^{2}\right)$ where depth(E) stands for the depth of the expression $E$ - note that the exact bound is $\left(2^{\operatorname{depth}(E)}-1\right) \times \mathrm{M}^{2}$. However, such an analysis does not help finding precisely which part of the code is responsible for most of the cost. Indeed since all the predicates (eval/3, eval_add/4, and eval_mult/4) are mutually recursive, the system will infer a similar cost for eval_add/4 and eval_mult/4. Furthermore, those costs will be expressed in terms of different input variables making the actual comparison difficult.

\subsection{Parametric Accumulated-Cost Static Profiling}

We now formalize the new notion of cost that we propose, the accumulated cost, which has been intuitively described in Section 4.1. As mentioned before, our approach is based on the notion of cost centers: user-defined program points (predicates, in our case) to which execution costs are assigned during the execution of a program. Data 
about computational events is accumulated by the cost center each time the corresponding program point is reached by the program execution control flow.

We start by presenting a formal profiled semantics for Logic Programming. For this purpose we assume given a program $\mathcal{P}$. We also assume that each predicate $p$ is associated with a cost $\operatorname{cost}_{\mathrm{p}} \in \mathbb{R}$ and that the cost centers are defined as a set $\diamond$ of predicate symbols. In the following we will use overlined symbols such as $\bar{\epsilon}, \bar{x}$, or $\bar{e}$ to denote a sequence of terms, variables, or arithmetic expressions.

We define a predicate call with context as a tuple of the form $r: p(\bar{t})$, where $r$, the context, is a cost center (i.e., a predicate from $\diamond$ ) and $\mathrm{p}(\overline{\mathrm{t}})$ is a predicate call. Then, we define profiled states as tuples of the form $\langle\alpha ; \theta ; \kappa\rangle$ where $\alpha$ is a sequence of predicate calls with context, $\theta$ is a substitution that maps variables to calling data, and $\kappa$, the cost assignment, is a family of real numbers indexed by the cost centers $\diamond$. The profiled resource semantics is defined as the smallest relation $\rightarrow_{P}$ over profiled states satisfying:

$$
\begin{gathered}
\frac{q=\text { update }_{\diamond}(\mathrm{p}, r) \quad(\mathrm{p}(\overline{\mathrm{s}}):-\beta) \in \mathcal{P} \rho \quad \sigma \text { is an m.g.u. of } \bar{s} \text { and } \overline{\mathrm{t} \theta}}{\langle r: \mathrm{p}(\overline{\mathrm{t}}), \alpha ; \theta ; \kappa\rangle \rightarrow_{\mathcal{P}}\left\langle\mathrm{q}: \beta, \alpha ; \theta \circ \sigma ; \kappa\left[q \mapsto \kappa_{\mathrm{q}}+\operatorname{cost}_{\mathrm{p}}\right]\right\rangle} \\
\frac{\sigma \text { is an m.g.u. of } \mathrm{t} \text { and }[\mathrm{s} \theta]}{\langle\mathrm{r}:(\mathrm{t} \text { is } \mathrm{s}), \alpha ; \theta ; \kappa\rangle \rightarrow_{\mathcal{P}}\langle\alpha ; \theta \circ \sigma ; \kappa\rangle}
\end{gathered}
$$

where:

- $q: \beta, \alpha$ is a notation for the sequence $q: p_{1}\left(\bar{s}_{1}\right), \ldots, q: p_{n}\left(\bar{s}_{n}\right), \alpha$, assuming $\beta$ is the sequence $\mathrm{p}_{1}\left(\mathrm{~s}_{1}\right), \ldots, \mathrm{p}_{n}\left(\mathrm{~s}_{n}\right)$.

- [s] stands for the arithmetic evaluation of $\mathrm{s}$ (if $\mathrm{s}$ is not a ground arithmetic expression, then $[\mathrm{s}]$ is not defined, as well as the rule using it),

- $\rho$ stands for a renaming with fresh variables,

- $\kappa[q \mapsto c]$ is the assignment that maps $\mathrm{p}$ to $c$ if $\mathrm{p}=\mathrm{q}$ or to $\kappa_{\mathrm{p}}$ otherwise, and

- $\operatorname{update}_{\diamond}(p, r)$ equals either $p$ if $p \in \diamond$, or $r$ otherwise.

The first rule can be understood as an extension of SLD resolution with cost. Concretely, the cost $\operatorname{cost}_{\mathrm{p}}$ of the called predicate $\mathrm{p}$ is added to the value of the current cost center, the cost center being updated beforehand to the current predicate if the latter is in fact a cost center, and left unchanged otherwise. The latter rule characterizes the semantics of the built-in is/2, where we assume w.l.o.g. that the operation has no cost. Standard left-to-right evaluation is simply recovered by ignoring the cost assignment together with the calling contexts. In the following section, we will use the notation $(\alpha ; \theta)$, where $\alpha$ is a sequence of predicate calls and $\theta$ a substitution, to denote a standard (non-profiled) LP state.

In the following, we use $\Pi$ as the set of tuples of terms, and $\mathbb{R}$ to denote the set of real numbers. For any cost center $\mathrm{p} \in \diamond$, the profiled resource usage function is the 
function $\mathcal{C}_{\diamond}^{\mathrm{p}}: 2^{\Pi} \rightarrow 2^{\mathbb{R}^{n}}$ defined as:

$$
C_{\diamond}^{\mathrm{p}}(\bar{T})= \begin{cases}\left\{\kappa \mid \overline{\mathrm{t}} \in \bar{T} \&\langle\mathrm{p}: \mathrm{p}(\overline{\mathrm{t}}) ; \varepsilon ; \overline{0}\rangle \rightarrow_{\mathcal{P}}^{*}\langle\square ; \theta ; \kappa\rangle\right\} & \text { if } \mathrm{p}(\overline{\mathrm{t}}) \text { terminates } \\ \mathbb{R}^{n} & \text { universally } \forall \overline{\mathrm{t}} \in \bar{T}\end{cases}
$$

where $\overline{0}$ stands for the trivial cost assignment that maps any cost center to $0, \rightarrow_{P}^{*}$ is the reflexive and transitive closure of $\rightarrow_{\mathcal{P}}, \square$ denotes the empty sequence of predicate calls, $\varepsilon$ is the identity substitution, and $n$ is the number of cost centers. We use the "top" element in $2^{\mathbb{R}^{n}}$ (i.e., $\mathbb{R}^{n}$ ) to denote a "don't know" cost for non-terminating programs, which, for simplicity, are currently not defined in our framework. Note that the cost $\kappa_{\mathrm{p}}$ in an infinite derivation can be (asymptotically) different from $+\infty$ as (1) p can be the context of only a finite number of the steps involved in an infinite derivation, and (2) because costs of predicates can be zero or negative. The profiled semantics is a natural generalization of the standard resource usage semantics which is able to handle several costs which are accumulated in the cost centers. Indeed the resource usage function inferred by the standard analysis can be understood as the function $C^{\mathrm{p}}=C_{\{\mathrm{p}\}}^{\mathrm{p}}$ defined over a unique cost center.

$C_{\mathrm{q}}^{\mathrm{p}}(\bar{T})$ denotes the cost accumulated in $\mathrm{q}$ from the calls $\mathrm{p}(\overline{\mathrm{E}})(\overline{\mathrm{E}} \in \bar{T})$, that is, the union of the $i^{\text {th }}$ component of all tuples in $\mathcal{C}_{\diamond}^{\mathrm{p}}(\bar{T})$ if $q$ is the $i^{\text {th }}$ cost center in $\diamond$ (formally $\mathcal{C}_{\mathrm{q}}^{\mathrm{p}}(\bar{T})=\left\{\kappa_{\mathrm{q}} \mid \kappa \in \mathcal{C}_{\diamond}^{\mathrm{p}}(\bar{T})\right\}$ ). In particular, if $\mathrm{p}(\overline{\mathrm{t}})$ deterministically succeeds (e.g., when it is obtained by translation of some imperative program) the cost accumulated in $\mathrm{q}$ from $\mathrm{p}(\overline{\mathrm{t}})$ is unique, i.e., $\mathcal{C}_{\mathrm{q}}^{\mathrm{p}}(\{\overline{\mathrm{E}}\})=\{c\}$ for some $c \in \mathbb{R}$. In such a case, by a slight abuse of notation, we denote the unique value by $C_{\mathrm{q}}^{\mathrm{p}}(\overline{\mathrm{t}})$.

Consider the deterministic program given in section 4.3. If we profile the program, defining all the predicates of the program as cost centers except add/4 and mult/4, the costs accumulated in eval_const/3, eval_add/4 and eval_mult/4 for a call of the form eval $(E, M, R)$ are respectively bounded by $0,\left(0.5 \times 2^{\operatorname{depth}(E)} \times M\right)$, and $\left(0.5 \times 2^{\text {depth }(E)} \times \mathrm{M}^{2}\right)$. This makes it easier to spot the source of most of the cost, i.e., eval_mult/4. Therefore, to improve the efficiency of the whole program, it can be useful to concentrate on this predicate, either by optimizing its implementation or by reducing the number of times it is called.

We write $p \rightsquigarrow q$ if $q$ is reachable from $q$, that is, if $q(\bar{t}) \rightarrow_{P}^{*}(p(\bar{s}), \alpha)$ for some calling data $\bar{t}$ and $\bar{s}$, and some sequence of calls $\alpha$. Given a set $\diamond$ of cost centers assigned to a program $\mathcal{P}$ and some predicate $\mathrm{p}$, we define the set of reachable cost centers from $p$ as the sequence $\nabla_{p}=\left\{q \mid q \in \diamond \wedge p \rightsquigarrow^{\star} q\right\}$.

Theorem 1. Let $\mathcal{P}$ be a program and $\diamond \subseteq \operatorname{pred}(\mathcal{P})$ a set of cost centers for it. Then, for all $\mathrm{p} \in \diamond$ : for all $\bar{T} \subset \Pi$ it holds that: $\mathcal{C}_{\mathrm{p}}(\bar{T})=\left\{\sum_{\mathrm{q} \in \diamond_{\mathrm{p}}} C_{\mathrm{q}}^{\mathrm{p}}(\bar{T})\right\}$. In particular, if $\mathrm{p}(\overline{\mathrm{E}})$ deterministically succeeds $\mathcal{C}_{\mathrm{p}}(\overline{\mathrm{E}})=\sum_{\mathrm{q} \in \diamond_{\mathrm{p}}} C_{\mathrm{q}}^{\mathrm{p}}(\overline{\mathrm{E}})$.

Note that theorem 1 provides the basis for a compositional and modular definition of the standard (i.e., single call) cost analysis, from the results of the accumulated cost analysis. Note also that (by definition of reachable cost center) $p$ is always reachable from itself, even though $\mathrm{p}$ does not call itself. 


\subsection{Inferring Accumulated Cost via Transformation}

As mentioned before, our implementation of the static profiler is based on a sourceto-source transformation. In this section we show such a transformation that allows obtaining accumulated cost information for cost centers by performing a sized type analysis in CiaoPP. Basically, the transformation consists of adding shadow arguments to each predicate of the Horn clauses that represent the accumulated cost for each cost center.

\subsubsection{The Transformation}

In this section we assume there is exactly $n$ cost centers and $\diamond$ is defined as the family $\left\{\mathrm{p}_{i}\right\}_{i \in 0 . . n-1}$. The transformation proposed consists of adding $n+1$ shadow arguments to each predicate, such that on success those variables will be assigned to the costs accumulated in the program. There are $n$ shadow arguments for the cost accumulated in the cost centers called by the predicate, and an additional one for the cost associated with the calling context, which is not known statically.

Formally, the transformation is defined by the functions $\llbracket \cdot \rrbracket_{\diamond}$ and $\llbracket \cdot \rrbracket_{n}$ that respectively translate clauses and goals. The function $\llbracket \cdot \rrbracket_{n}: \mathcal{A}^{*} \rightarrow\left(\mathcal{A}^{*} \times E^{n+1}\right)(E$ is the set of possibly non-ground arithmetic expressions) that translates sequences of atoms is defined recursively on the length of the goal as:

- $\llbracket q(\bar{t}), \alpha \rrbracket_{n}=((q(\bar{t}, \bar{x}), \beta), \bar{x}+\bar{e})$ where $(\beta, \bar{e})=\llbracket \alpha \rrbracket_{n}$

- $\llbracket \square \rrbracket_{n}=(\square, \overline{0})$

where $\overline{\mathrm{x}}($ resp. $\overline{0})$ stands for a sequence of $(n+1)$ fresh variables (a sequence of $(n+1)$ zeros). On the other hand the function $\llbracket \cdot \rrbracket_{\diamond}: C \rightarrow C$ is defined by cases as follows:

$$
\llbracket q(\bar{t}):-\alpha \rrbracket_{\diamond}=\left\{\begin{aligned}
(q(\bar{t}, \bar{x}):-\beta, & \\
\bar{x} \text { is } \bar{e}\left[\overline{\mathrm{e}}_{n} \leftarrow 0\right]\left[\overline{\mathrm{e}}_{i} \leftarrow\left(\operatorname{cost}_{\mathrm{q}}+\mathrm{e}_{i}+\mathrm{e}_{n}\right)\right] & \text { if } q=\mathrm{p}_{i} \in \diamond \\
(\mathrm{q}(\overline{\mathrm{E}}, \overline{\mathrm{x}}):-\beta, & \\
\overline{\mathrm{x}} \text { is } \overline{\mathrm{e}}\left[\overline{\mathrm{e}}_{n} \leftarrow\left(\operatorname{cost}_{\mathrm{q}}+\mathrm{e}_{n}\right)\right] & \text { otherwise }
\end{aligned}\right.
$$

where $(\beta, \overline{\mathrm{e}})=\llbracket \alpha \rrbracket_{n}, \overline{\mathrm{x}}$ is a sequence of $n+1$ fresh variables, and $\overline{\mathrm{x}}$ is $\overline{\mathrm{e}}$ is a notation for $\mathrm{x}_{0}$ is $\mathrm{e}_{0}, \ldots, \mathrm{x}_{n}$ is $\mathrm{e}_{n}$ (assuming $\overline{\mathrm{x}}=\left(\mathrm{x}_{0}, \ldots, \mathrm{x}_{n}\right)$ and $\overline{\mathrm{e}}=\left(\mathrm{e}_{0}, \ldots, \mathrm{e}_{n}\right)$ ).

The translation of a clause is defined by case on the predicate $q$ it defines. Suppose $q$ is some cost center $p_{i} \in \diamond$. In this case the costs associated with $q$ itself (i.e., $\operatorname{cost}_{\mathrm{q}}$ ) are assigned to the argument corresponding to $q$, namely $e_{i}$. Furthermore the costs in evaluating $q$ that are not associated to any other cost center (i.e., $e_{n}$ ) are also assigned to $e_{i}$. Thus we have $\bar{e}\left[\bar{e}_{n} \leftarrow 0\right]\left[\bar{e}_{i} \leftarrow\left(\operatorname{cost}_{q}+e_{i}+e_{n}\right)\right]$. On the other hand, if $q$ is not a cost center, then the costs associated with $q$ are associated to its context, namely $e_{n}$, and thus we have $\bar{e}\left[\bar{e}_{n} \leftarrow\left(\operatorname{cost}_{\mathrm{q}}+e_{n}\right)\right]$. 
Example 1. We show now the translation of the code corresponding to our running example, given in Example 4.3, assuming that the cost centers are eval/3, eval_const/4, eval_add/4, and eval_mult/4. In the translation the output arguments $\mathrm{Ce}, \mathrm{Cc}, \mathrm{Ca}$, and $\mathrm{Cm}$ correspond to the cost accumulated in the respective cost centers. On the other hand, the output $\mathrm{C}$ is the cost that has not been accumulated in any of the cost centers. Within the translation we leave the actual implementations of add/4 and mult / 4 unspecified and marked by (...).

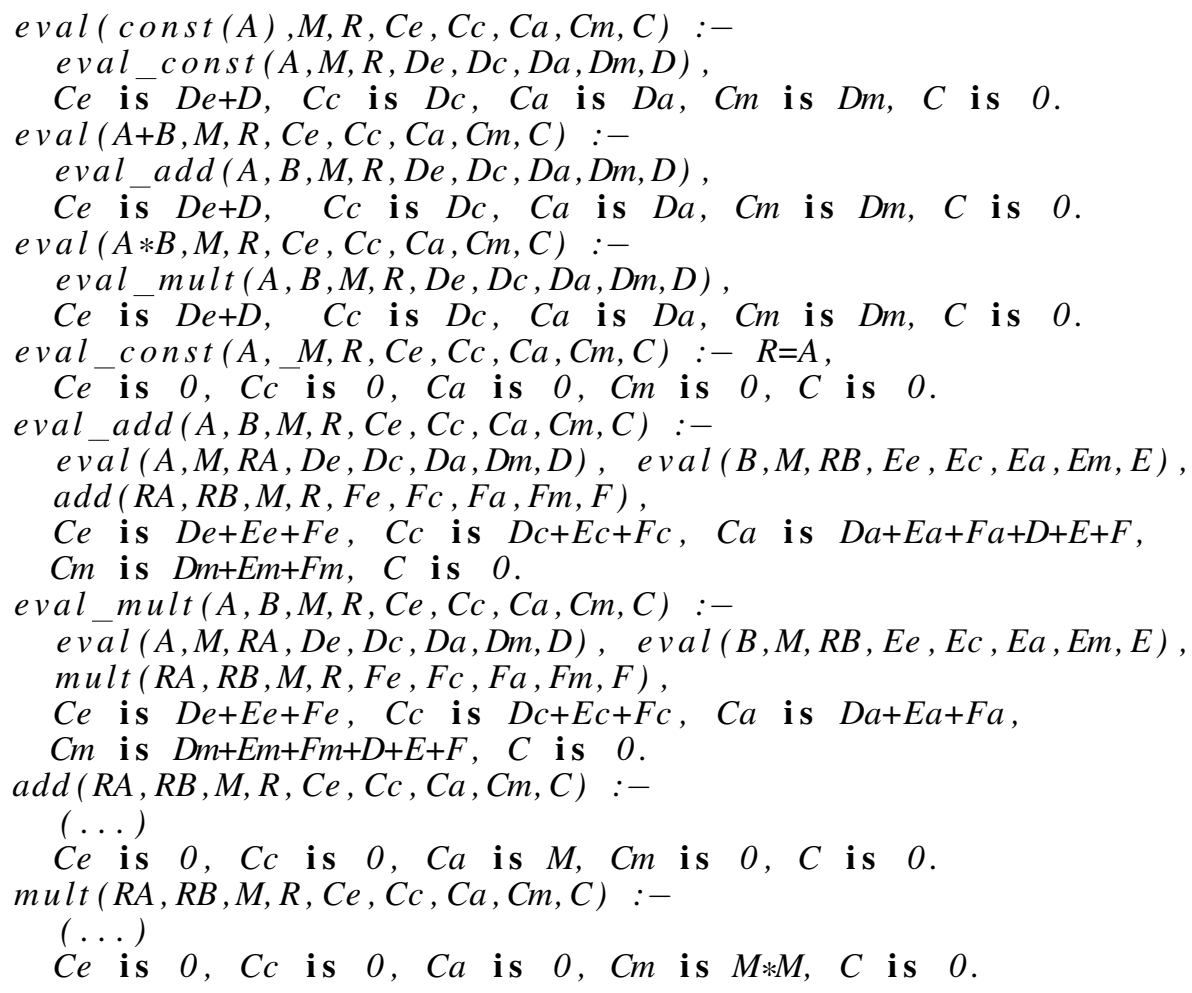

The following theorem states that the translation of a given program simulates the original one, while reifying the cost assignment as a first-order argument.

Theorem 2. Assume a given program $\mathcal{P}$ profiled according $n$ cost centers $\diamond=\left\{\mathrm{p}_{0}, \ldots, \mathrm{p}_{n-1}\right\}$ and a predicate $\mathrm{p}$ different from is.

(Soundness) If $(\mathrm{p}(\overline{\mathrm{t}}, \overline{\mathrm{x}}) ; \theta) \rightarrow_{\llbracket \mathcal{P} \rrbracket_{\diamond}}^{*}(\square ; \sigma)$ (for some sequence of pairwise $\bar{x}$ distinct variables free in $\bar{t}$ and $\theta)$ then there exists a derivation of the form $\left\langle\mathrm{p}_{i}: \mathrm{p}(\overline{\mathrm{t}}) ; \theta ; \overline{0}\right\rangle \rightarrow_{\mathcal{P}}^{*}$ $\left\langle\square ; \sigma^{\prime} ; \kappa\right\rangle$, with $\overline{\mathrm{t}} \sigma^{\prime}=\overline{\mathrm{t}} \sigma, \kappa_{\mathrm{p}_{j}}=\mathrm{x}_{j} \sigma($ for $j \in 1, \ldots, n-1$ and $j \neq i)$, and $\kappa_{i}=$ $\mathrm{x}_{i} \sigma+\mathrm{x}_{n} \sigma$.

(Completeness) If $\left\langle\mathrm{p}_{i}: \mathrm{p}(\overline{\mathrm{t}}) ; \varepsilon ; \overline{0}\right\rangle \rightarrow_{P}^{*}\langle\square ; \theta ; \kappa\rangle$, then there exists a derivation of the form $(\mathrm{p}(\overline{\mathrm{t}}, \overline{\mathrm{x}}) ; \varepsilon) \rightarrow_{\llbracket \mathcal{P} \rrbracket_{\diamond}}^{*}(\square ; \sigma)$, with $\overline{\mathrm{t}} \theta=\overline{\mathrm{t}} \sigma, \kappa_{\mathrm{p}_{k}}=\mathrm{x}_{j} \sigma($ for $j \in 1, \ldots, n-1$ and $j \neq i)$, and $\kappa_{i}=\mathrm{x}_{i} \sigma+\mathrm{x}_{n} \sigma$. 


\subsubsection{Performing the Resource Usage Analysis}

The Horn Clause program resulting from the transformation described above, whose predicates are augmented with shadow output arguments representing the accumulated cost for each cost center, is analyzed in order to infer lower and upper bounds on the sizes of such arguments, which actually represent bounds on the respective accumulated costs.

In order to obtain such bounds, we use the size analysis presented in [86, 87], integrated in the CiaoPP system. The goal of this analysis is to infer lower and upper bounds on the sizes of output arguments as a function on the sizes of input arguments. This analysis is based on the abstract interpretation framework present in CiaoPP, and basically infers sized types for output arguments. Sized types are representations that incorporate structural (shape) information and allow expressing both lower and upper bounds on the size of a set of terms and their subterms at any position and depth. For a more detailed explanation of this process, we refer the reader to [86].

Continuing with our running example, consider the output argument $\mathrm{Ca}$, which represents the accumulated cost of the cost center eval_add/ 4 when it is called from eval/4. In a preprocessing step, the program is unfolded in order to avoid mutual recursion, which makes the analysis harder. After the unfolding step, the analysis infers types for the predicate arguments by using an existing analysis for regular types [93]. This analysis infers that for a call to a transformed version of eval/4 (with shadow variables) of the form:

$$
\text { eval (Exp, M, R, Ce, Cc, Ca, Cm, C) }
$$

with Exp and $\mathrm{R}$ bound and the rest of arguments as free variables, then $\mathrm{Ca}$ gets bound to a number upon success, i.e., a term of type num. From the inferred regular type, the analysis derives a sized type schema, which is just a sized type with variables in bound positions, along with a set of constraints over those variables.

In this case, the corresponding sized type for num is num ${ }^{(\alpha, \beta)}$, where $\alpha$ and $\beta$ are variables representing lower and upper bounds on the size of the elements that belong to such type. The metric we use for the size of a number is its actual value, since num is a basic type. For compound types, e.g., lists, trees or arithmetic expressions, we can use several metrics for the size of any term belonging to them, such as the depth of such term (as in our example), or the number of type rule applications needed for the type definition to succeed for such term.

The next step involves setting up recurrence relations between size variables. Thus, for $\beta$, that represents the upper bound of the size of $\mathrm{Ca}$, we obtain the following equation (where Size $e_{\text {arg }}^{\text {pred }}$ is the size of the argument arg corresponding to predicate pred):

$$
\beta=\operatorname{Size}_{\mathrm{Ca}}^{\text {eval }}\left(\text { Size }_{\text {exp }}, M\right)= \begin{cases}2 * \operatorname{Size}_{\mathrm{Ca}}^{\text {eval }}\left(\text { Size }_{\text {exp }}-1, M\right)+M & \text { if Size } e_{\text {exp }}>1 \\ 0 & \text { otherwise }\end{cases}
$$

At this point, we have obtained a recurrence relation that represents the size of the output argument. However, such expression is not useful for some applications. 
One disadvantage of using recurrence relations is that the evaluation of them given concrete input values usually takes longer than the evaluation of an equivalent nonrecursive expression. In addition, it is not easy to see the complexity order of a given procedure just by looking at its recurrence relation, and the comparison with other functions is also more difficult. For this reason, the analysis uses a solver for obtaining closed-form representations for recurrence relations. Such closed forms can be either exact solutions or safe overapproximations. In our example, the closed-form version for the recurrence is:

$$
\beta=\operatorname{Size}_{C a}^{\text {eval }}\left(\text { Size }_{\text {exp }}, M\right)=\left(2^{\text {Size }_{\text {exp }}}-1\right) * M
$$

Assuming that the metric for the size of arithmetic expressions is the depth of the term representing them, we have that $S_{i z e_{\exp }}=\operatorname{depth}(\exp )$. Thus, we can finally conclude that the accumulated cost of eval_add/ 4 when called from eval/3 (i.e., the size of $\mathrm{Ca}$ in the transformed version of the program), is given by

$$
\left(2^{\operatorname{depth}(\exp )}-1\right) * M
$$

\subsection{Experimental Results}

We have performed an experimental evaluation of our techniques with the prototype implementation described in Section 4.5 over a number of selected benchmarks from [87]. The benchmarks are written directly as Horn Clause programs (in Ciao). In each benchmark, a number of predicates are marked as cost centers. The results are shown in Table 4.1. Static profiling was performed for each cost center, capturing the accumulated cost with respect to an entry predicate (marked with a star, e.g., appendAll2*). While in the experiments both upper and lower bounds were inferred, for the sake of brevity we only show upper bound functions. Also, each clause body is assumed to have unitary cost.

Column 1 of Table 4.1 shows the list of benchmarks while column 2 provides the list of cost centers for each benchmark. Column 3 shows the parametric accumulated cost inferred for each cost center, as a resource usage upper bound function on input data sizes of the entry predicate.

Column 4 compares the parametric accumulated cost function of each cost center from column 3 with the results from a dynamic profiling tool [69]. Although the analysis infers upper bounds on the accumulated cost, for some benchmarks these are exact upper bounds (in fact, exact costs) and for others these are correct but relatively imprecise. The imprecision introduced in the benchmarks listfact and appendAll2 is due to the fact that the cost not only depends on the input data sizes but also on the sizes of the sub-terms in the input data, since the analysis statically assumes an upper bound on the sizes of the sub-terms. Note that CiaoPP is the only analysis tool that infers concrete upper bound functions over sized types (costs that depend on the sizes of subterms) [87]. 
Chapter 4. Transformational Approach to Parametric Accumulated-Cost Static Profiling

Table 4.1: Experimental results.

\begin{tabular}{|c|c|c|c|c|c|}
\hline Program & $\begin{array}{l}\text { Cost- } \\
\text { Center } \\
\text { Predicate } \\
\end{array}$ & $\begin{array}{l}\text { Accumulated } \\
\text { Cost UB }\end{array}$ & $\begin{array}{l}\text { Static } \\
\text { vs. } \\
\text { Dyn } \\
\end{array}$ & $\begin{array}{l}\text { Standard } \\
\text { Cost UB }\end{array}$ & \#Calls \\
\hline appendAll2 & $\begin{array}{l}\text { appendAll2* } \\
\text { appendAll } \\
\text { append }\end{array}$ & $\begin{array}{l}b_{1} \\
b_{1} b_{2} \\
2 b_{1} b_{2} b_{3}\end{array}$ & $\begin{array}{l}0 \% \\
33 \% \\
61 \%\end{array}$ & $\begin{array}{l}2 b_{1} b_{2} b_{3}+ \\
b_{1} b_{2}+b_{1} \\
b_{1} b_{2} \\
\beta\end{array}$ & $\begin{array}{l}1 \\
b_{1} \\
b_{1} b_{2}+b_{1}\end{array}$ \\
\hline hanoi & $\begin{array}{l}\text { hanoi }^{*} \\
\text { processMove }\end{array}$ & $\begin{array}{l}2^{v}-1 \\
2^{v}-1\end{array}$ & $\begin{array}{l}0 \% \\
0 \%\end{array}$ & $\begin{array}{l}2^{v+1}-2 \\
1\end{array}$ & $\begin{array}{l}1 \\
2^{v}-1\end{array}$ \\
\hline coupled & $\begin{array}{l}\text { coupled }^{*} \\
f \\
g\end{array}$ & $\begin{array}{l}1 \\
\frac{v}{2}+\frac{(-1)^{v}}{4}+\frac{3}{4} \\
\frac{v}{2}+\frac{(-1)^{v}}{4}-\frac{1}{4}\end{array}$ & $\begin{array}{l}0 \% \\
1.2 \% \\
0 \%\end{array}$ & $\begin{array}{l}v+1 \\
v \\
v\end{array}$ & $\begin{array}{l}1 \\
\frac{v}{2}-\frac{(-1)^{v}}{4}+ \\
\frac{1}{4} \\
\frac{v}{2}+\frac{(-1)^{v}}{4}- \\
\frac{1}{4}\end{array}$ \\
\hline minsort & $\begin{array}{l}\text { minsort } \\
\text { findmin }\end{array}$ & $\begin{array}{l}\beta+1 \\
\frac{(\beta+1)^{2}}{2}+\frac{\beta-1}{2}\end{array}$ & $\begin{array}{l}0 \% \\
7 \%\end{array}$ & $\begin{array}{l}\frac{(\beta+1)^{2}}{2}+\frac{\beta+1}{2} \\
\beta\end{array}$ & $\begin{array}{l}1 \\
\beta+1\end{array}$ \\
\hline dyade & $\begin{array}{l}\text { dyade* } \\
\text { mult }\end{array}$ & $\begin{array}{l}\beta_{1} \\
\beta_{1} \beta_{2}\end{array}$ & $\begin{array}{l}0 \% \\
0 \%\end{array}$ & $\begin{array}{l}\beta_{1}\left(\beta_{2}+1\right) \\
\beta\end{array}$ & $\begin{array}{l}1 \\
\beta_{1}\end{array}$ \\
\hline $\begin{array}{l}\text { variance } \\
\text { naive }\end{array}$ & $\begin{array}{l}\text { variance }^{*} \\
\text { sq_diff } \\
\text { mean }\end{array}$ & $\begin{array}{l}1 \\
\beta-1 \\
2 \beta^{2}-\beta\end{array}$ & $\begin{array}{l}0 \% \\
0 \% \\
0 \%\end{array}$ & $\begin{array}{l}2 \beta^{2} \\
2 \beta_{2} \beta_{1}-2 \beta_{2} \\
\beta-1\end{array}$ & $\begin{array}{l}1 \\
\beta-1 \\
\beta\end{array}$ \\
\hline variance & $\begin{array}{l}\text { variance }^{*} \\
\text { sq_diff } \\
\text { mean }\end{array}$ & $\begin{array}{l}1 \\
\beta \\
4 \beta+2\end{array}$ & $\begin{array}{l}0 \% \\
0 \% \\
0 \%\end{array}$ & $\begin{array}{l}5 \beta+3 \\
\beta \\
2 \beta+1\end{array}$ & $\begin{array}{l}1 \\
\beta \\
2\end{array}$ \\
\hline listfact & $\begin{array}{l}\text { listfact } \\
\text { fact }\end{array}$ & $\begin{array}{l}\beta \\
\beta \delta+\beta\end{array}$ & $\begin{array}{l}0 \% \\
47 \%\end{array}$ & $\begin{array}{l}\beta(\delta+2) \\
\delta+1\end{array}$ & $\begin{array}{l}1 \\
\beta\end{array}$ \\
\hline
\end{tabular}

- $\ln { }^{\left(\alpha_{i}, \beta_{i}\right)}\left(n^{\left(\gamma_{i}, \delta_{i}\right)}\right)$ represents the size of the list of numbers $L_{i}$, where $\beta_{i}$ and $\delta_{i}$ (resp. $\alpha_{i}$ and $\gamma_{i}$ ) denote the upper (resp. lower) bounds on the length of the list and the size of its numbers respectively.

- $l l l n^{\left(a_{1}, b_{1}\right)}\left(l \ln { }^{\left(a_{2}, b_{2}\right)}\left(\ln ^{\left(a_{3}, b_{3}\right)}\left(n^{\left(a_{4}, b_{4}\right)}\right)\right)\right)$ represents the size of the list of lists of lists of numbers similarly.

- $n^{(\mu, v)}$ denotes the size of a number with lower- and upper-bounds $\mu$ and $v$ respectively.

Column 5 shows for comparison the cost inferred by the standard (i.e., non-accumulated) cost analysis [87] for each program and its auxiliary predicates (also marked as cost centers). The comparison of the accumulated and standard cost functions (columns 3 vs. 5) shows the usefulness of our approach: the upper bounds on cost centers display accumulated costs for program parts that were not visible with the standard analysis. 
For instance, similarly to Example 4.3, the coupled benchmark has two auxiliary mutually recursive predicates $f$ and $g$ that are processing elements of a list alternatively until the list becomes empty. The standard analysis infers almost the same upper bound for both functions due to the mutual recursion, whereas the accumulated cost precisely points out the source of cost in the mutually recursive parts. Similarly, in hanoi, although the cost of processMove (processing a single hanoi move) is unitary, we can see that it is called an exponential number of times. The analysis is providing hints to the programmer about the parts of the program that are most profitable candidates for optimization. Note that the upper bound cost functions inferred by static profiling for each cost center predicate are on the input data sizes of the program (entry predicate), in contrast to the standard analysis where the cost functions are on the input data sizes of the predicate that the cost function corresponds to.

Finally, in column 6 an additional \#Calls cost is presented, indicating the number of times each predicate is called, as a function of input data sizes of the entry predicate. These cost functions are inferred using the standard analysis by defining explicitly a \#Calls resource for each cost center predicate. A big complexity order in the number of calls to a predicate (in relation to that of a single call) might give hints to reduce the number of calls to such predicate in order to effectively reduce its impact on the overall cost of the program (i.e., the cost of a call to the entry point). More interestingly, since both the Accumulated and \#Calls costs of a predicate $q$ are expressed as functions of input data sizes of the entry predicate, their quotient (Column 3 / Column 6) is meaningful and will give an approximation of the cost of a single call to $q$ as a function of the input data sizes of the entry predicate. Note that the standard analysis (Column 5) also provides an upper-bound approximation of this cost but as a function of the input data sizes of predicate $q$.

\subsection{Related Work}

Static profiling in the context of Worst Case Execution Time (WCET) Analysis of real-time programs is presented in [15]. It proposes an approach to computing worstcase timing information for all code parts of a program using a complementary metric, called criticality. Every statement of a real-time program is assigned with a criticality value, expressing how critical the respective code is for the global WCET. Our approach is not limited to WCET, since it is able to obtain results for a general class of user-defined resources. Furthermore, our inferred metrics are parametric on the input data sizes of the main program, in contrast to the criticality metric, which is a numeric value in the range $[0,1]$. In addition, our approach is modular and compositional, able to compute accumulated costs w.r.t. calls originating from different procedures of the program, and not only the main program entry point. In [13] the authors present static profiling techniques to estimate the execution likelihood and frequency of program points in order to assess whether the cost of certain compile-time optimizations would pay off. To this end, they explore the use of some static analysis techniques for predicting the result of conditional branches, such as assuming uniform distribution over 
all branches, making heuristic based predictions, and performing value range propagation. In this context, our approach can be used to infer bounds on the number of times a certain program point will be called from a given entry point, as functions on input data sizes, in contrast with a single value representing the execution likelihood or frequency. Besides, since our techniques are supported mainly by the theory of abstract interpretation, the approximations inferred are correct by design.

\subsection{Chapter Conclusions}

In this chapter we have presented a novel approach of static profiling of accumulated cost that infers upper- and lower-bounds of the resource usage accumulated in particular parts of a program as a functions on the input data sizes of the program. We have constructed a prototype implementation of the proposed approach using the CiaoPP program analysis framework. Preliminary experimental results with the tool support the usefulness of our approach where precise accumulated upper bound cost functions were inferred for parts of the program for which the standard analysis was not able to infer precise information. The upper bound functions inferred by the static profiling were also evaluated against a dynamic profiling tool [69], and showed promising accuracy for the static analysis. However in cases where the cost depended on the sizes of the sub-terms of the input, the upper bound accumulated cost loses precision. 



\section{Energy Efficient Scheduling}

\subsection{Introduction}

Energy efficient scheduling has gained a lot of interest in the recent past. A great number of publications, e.g., [32], try to present it as a mixed integer linear optimisation problem, which can be solved using mixed integer linear programming, or using a heuristic approach. However, these algorithms become impractical or fail to deliver a solution as the problem size grows. There is a significant group of publications on using Evolutionary Alogrithms (EAs) for the problem of optimal scheduling and allocation in multiprocessor systems that allow Dynamic Voltage and Frequency Scaling (DVFS), e.g., the approach presented in [70] aims to minimize both energy and makespan as a bi-objective problem.

Energy efficient scheduling and allocation in multicore environments with enabled DVFS is a well-known $N P$-hard problem. Nevertheless approximated solutions can be efficiently found by heuristic algorithms, such as evolutionary algorithms (EAs). However, these algorithms have some drawbacks that hinder their applicability: typically they are very slow, and if the space of the feasible solutions is too restricted, they often fail to provide a viable solution. To this end, in the first part of this chapter, in Section 5.2, we propose an approach that overcomes these issues. The approach is based on a custom EA that is fed with predicted information provided by the static analysis about the energy consumed by tasks. This solves the time inefficiency problem. In addition, when this algorithm fails to produce a feasible solution, we resort to a modification of the well-known YDS algorithm that we have performed, well adapted to the multicore environment and to the situations when the static power becomes the predominant part. This way, we propose a combined approach that produces an energy efficient scheduling in reasonable time, and always finds a viable solution. The approach has been tested on multicore XMOS chips, but it can easily be adapted to other multicore environments as well. In the tested scenarios the modified YDS can improve the original one up to $20 \%$, while our EA can save 55-90\% more energy on average than the modified YDS. 
The second part of the chapter (Section 5.3) addresses the problem of energy efficient scheduling and allocation of tasks in multicore environments, where the tasks can permit certain loss in accuracy of either final or intermediate results, while still providing proper functionality. Loss in accuracy is usually obtained with techniques that decrease computational load, which can result in significant energy savings. To this end, in this work we use perforation, which consists in skipping every $n$-th loop iterations for a given $n$ the loop perforation technique that transforms loops to execute a subset of their iterations, and integrate it in our optimisation tool for energy efficient scheduling in multicore environments based on evolutionary algorithms and static analysis for estimating energy consumption of different schedules. The approach is designed for multicore XMOS chips, but it can be adapted to any multicore environment with slight changes. The experiments conducted on a case study in different scenarios show that our new scheduler enhanced with loop perforation improves the previous one, achieving significant energy savings (31\% on average) for acceptable levels of accuracy loss.

\subsection{Energy Efficient Scheduling using Evolutionary and YDS Algorithms with Faster Energy Estimations}

In our setting, we want to solve the general scheduling problem where the tasks have arbitrary release times and deadlines, and where preemption and migration of tasks are allowed, but the problem still remains $N P$-hard for arbitrary release times and deadlines of the tasks which are not agreeable, ${ }^{1}$ as it was proven in [1]. Our algorithm has been adapted for its application to multicore XMOS chips, but it could easily be adapted to any multicore environment, ranging from small scale embedded systems up to large scale systems, such as data centers. Its first practical implementation relied on an existing analytical model for calculating the energy consumption for programs running on these chips [50]. The energy model is limited to the cases when all the cores belong to the same chip, thus they must run at the same voltage and frequency level at each moment. For this reason, in this work we solve the problem of the so-called global DVFS, when all the cores always have the same voltage and frequency.

In our first EA implementation, each individual in each generation is evaluated by using the above mentioned program energy model, which requires the execution traces of the programs. Given that the traces can be huge, even for small programs, such evaluation introduces a huge amount of overhead. In order to overcome this issue, in this section we use our static analysis which, at compile time, without the need of executing the programs, and in a few seconds, gives a safe estimation of the energy consumed by programs. As energy consumption often depends on (the size of) input data, which is not known at compile time, the static analysis provides the energy as a function of the input parameters, which is calculated once such input values are known at runtime. The energy consumption estimated by using the static analysis for a given

\footnotetext{
${ }^{1}$ Two tasks are agreeable if the task with later release time also has a later deadline.
} 
scheduling is computed as the sum of the energies of the tasks running on different cores. This gives a safe upper bound on the total energy consumption, although it may be less precise than the estimations computed with the program energy model mentioned previously. This may reduce possible energy savings, nevertheless, the information it provides is still good enough to decide which scheduling is better, and the gain in speed of the algorithm is huge: the simulation time is reduced from a few hours to a few minutes.

However, the EAs can have trouble in finding a viable solution, in the sense that not all task deadlines are met, if the task deadlines are too tight. In order to overcome this problem, we have adapted the standard YDS ${ }^{2}$ algorithm [102] (explained in Section 5.2.1) to multicore environments. As we will see later, our experimental results show that if the EA finds a viable solution, it is better than the one obtained by our modified YDS algorithm in terms of energy savings.

For these reasons, the approach we propose (depicted in Figure 5.1) consists of the following steps:

1. Perform the static analysis of the input tasks to estimate the energy consumed by each of them.

2. Execute the EA using such estimations.

- If the EA provides a viable solution, i.e., all the task deadlines are met, this is the final solution.

- Otherwise, execute our modified YDS algorithm and take its output as the final solution.

We can distinguish two energy models in Figure 5.1:

- Instruction-level energy model: gives an estimation of the energy consumed by the execution of a single instruction, which in general depends on its inputs, context, etc. However, for simplicity, the model assigns a constant value to each instruction. It is used by an abstract-interpretation based static analysis to infer the energy consumed by a program.

- Program-level energy model: a formula that gives the energy consumption of the entire program, as presented in [50], which is used by our modified YDS algorithm.

In summary, in this section we propose a time efficient scheduling approach, which always provides a viable energy efficient solution, and scales well as the input size grows. The main original contributions of our approach are:

1. The combination of an EA algorithm that resorts to a modified YDS algorithm, which always provides a viable solution.

\footnotetext{
${ }^{2}$ The name is created using the first letter of the authors' last names.
} 


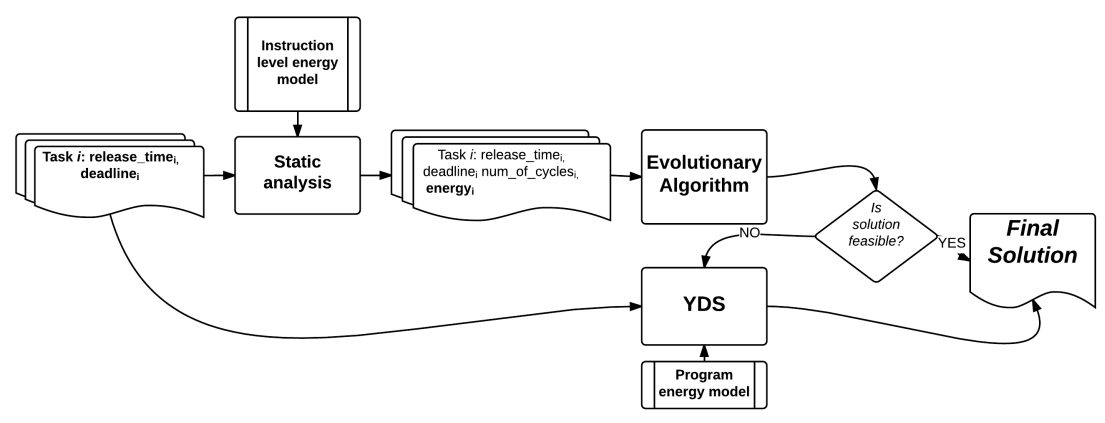

Figure 5.1: Overview of our scheduling approach.

2. Use of static analysis for energy estimation at compile time to guide the EA process, which results in significant speed-up in solving the scheduling problem, and hence the practicability of our approach, while still providing a solution with significant energy savings.

3. An improvement of the YDS algorithm, which efficiently solves the static power issue in the situations the chip cannot be switched off (explained in Section 5.2.1).

The rest of the section is organised as follows. Section 5.2.1 gives more details about our proposed approach. Section 5.2.2 presents an experimental evaluation of the approach. Finally, some conclusions are drawn in Section 5.2.3.

\subsubsection{Our Proposed Approach}

Evolutionary Algorithm The approach we propose is based on the NSGA-II [23] multiobjective evolutionary algorithm with two objectives: the execution time and the total energy consumption, where both should be minimised. The objectives are clearly in conflict, since the application of DVFS reduces energy, but increases execution time. This justifies the usage of a multiobjective algorithm. NSGA-II has been proven in the literature to perform good when the number of objectives is small [11], and in our case we have only two.

Since the output of the multiobjective approach is a set of solutions which form the (approximated) Pareto front, we can choose the solution that meets some given energy and/or time requirements. Usually we pick the solution with the minimal energy consumption among those that meet the given time bound (if applicable).

Individual Representation The problem that we are solving is the optimal (in terms of energy or time, possibly under some requirements involving them) allocation and scheduling of a set of tasks, where each task is defined by its:

- Unique $I D$.

- Release time, i.e., the moment when the task becomes available. 
- Deadline, i.e., the latest moment when the task has to finish.

- Number of clock cycles, as a good approximation of the execution time.

Thus, the solution to this problem has to contain the following information:

- The core(s) where each task will be executed. Since we allow task migration, a task can be allocated to more than one core.

- The current voltage and clock frequency $(V, f)$ state to exploit DVFS.

- The time periods when the tasks are executed.

- The number of clock cycles each task will execute in the different periods marked by the preemption and migration of that task. This allows to express the number of cycles a task will execute before it is preempted, as well as the number of cycles it will execute after it is resumed in the same or in a different core, etc.

Having in mind these requirements, we have designed the solution representation as shown in Figure 5.2, which does not introduce significant overhead when executing the EA. Any given task has a positive (unique) number as its ID. Each gene representing a task ID is followed by a gene representing the number of cycles of the task that will be executed without any preemption. The order of task IDs represents the order of their temporal execution. We also use negative two digit numbers to encode the spatial allocation of the tasks. The first digit represents the core where the tasks are being executed and the second one an encoding of the $(V, f)$ state of that core. As it will be explained in Section 5.2.2, Table 5.1, the number of different cores and states is finite, as well as the number of their combinations. The tasks following the allocation code are executed on that coded location. For instance, on Figure 5.2 we read: on core 1 in state 2,48 cycles of task 1 will be executed, and 77 cycles of task 5 , in this exact order, etc.

Our approach allows a random order of allocation codes, in order to solve the most general problem. However, if two consecutive allocation codes have different $(V, f)$ states, this means that the tasks allocated to the cores they represent will not be executed in parallel, since all of the cores have to run at the same $(V, f)$ at any moment, and thus the $(V, f)$ state has to be changed before the second group of tasks is executed. For example, in Figure 5.2 the allocation code following -12 is -24 , which means that the chip will be first in the $(V, f)$ state 2 and all tasks allocated to core 1 will be executed sequentially on that core. After they finish their execution, the core will change its $(V, f)$ state from 2 to 4 , and the tasks allocated to core 2 will be executed sequentially on core 2 . If the second allocation code were -22 instead of -24 , then the $(V, f)$ state would not change, and the tasks allocated on cores 1 and 2 would be executed in parallel.

Population Initialisation Individuals in the initial population are created by randomly assigning tasks to random cores in random $(V, f)$ settings with equal probability. However, in order to provide a load balanced solution (as much as possible), the probability 


\begin{tabular}{|c|c|c|c|c|c|c|c|}
\hline$\ldots$ & -12 & 1 & 48 & 5 & 77 & -24 & $\ldots$ \\
\hline & $\begin{array}{l}\text { Core 1, } \\
\text { state 2 }\end{array}$ & Task 1 & $\begin{array}{c}48 \\
\text { cycles } \\
\text { of task 1 }\end{array}$ & Task 5 & $\begin{array}{c}77 \\
\text { cycles } \\
\text { of task } 5\end{array}$ & $\begin{array}{l}\text { Core 2, } \\
\text { state 4 }\end{array}$ & \\
\hline
\end{tabular}

Figure 5.2: An example of (part of) a solution (i.e., individual) representation.

of choosing a core decreases as its load increases, which is given by the following formula:

$$
\text { Prob }=\frac{1}{\text { NumberOfCores }}-\frac{\text { CurrentCoreLoad }}{\text { TotalLoad }}
$$

where CurrentCoreLoad stands for the current load of the core expressed as the number of cycles, while TotalLoad stands for the total number of cycles of all the tasks on all cores. According to the formula, at the beginning of the initialisation process, all cores have the same probability of being chosen, while this probability decreases as the core becomes loaded, and is close to 0 when the load reaches the state where it is equally distributed in all cores. If during the initialisation process a newly calculated probability value of a core is below 0 , the value is rounded to 0 , and no new load will be assigned to that core. These random solutions do not always have to provide a viable solution, i.e., some of the tasks might miss their deadlines. For this reason, the mutation operator and the objectives are designed to deal with this problem.

Solution Perturbations Given the unique nature of the individual representation, we have designed new crossover and mutation operators. The individuals that participate in the crossover are selected by using the standard tournament selection process.

The Crossover Operator Since our solution allows task migration, a given task ID can appear more than once, so we cannot apply any of the existing permutation-based crossover operators. Thus, we have designed our own operator, where each child will preserves the order of genes representing the task allocation and scheduling from one parent, and only the genes representing the number of cycles can be taken from the other parent. In this way, the produced offspring is a combination of both parents, and is at the same time a viable solution to the problem. The process is depicted in Figure 5.3 for the most simple case of 2 cores, 2 tasks and 2 states, with one possible output. We can observe that the first child, $C 1$ takes the scheduling and allocation from the first parent, $P 1$, and the cycle distribution from the second parent, $P 2$, while the second, $C 2$, takes the scheduling and allocation from $P 2$ and the cycle distribution from $P 1$.

The Mutation Operator The mutation operator can perform different actions involving one or two tasks. Consider two tasks $i$ and $t$. When choosing the first one we give higher probability to the tasks which miss their deadlines, in order to achieve a viable 


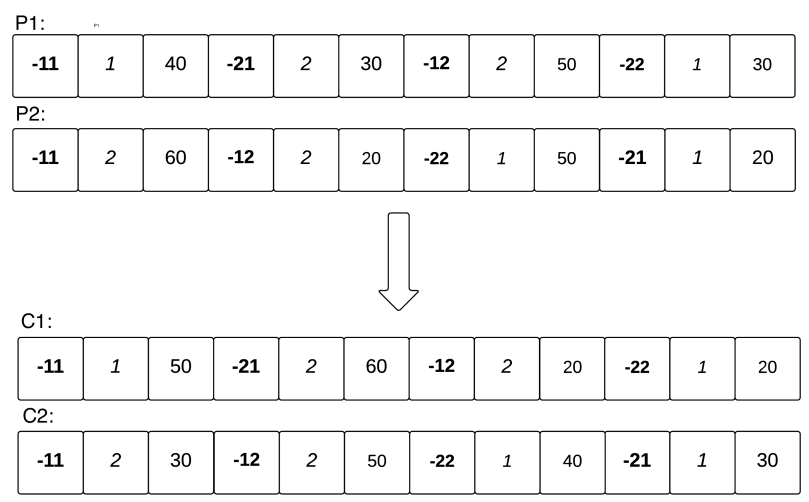

Figure 5.3: An example of a crossover operation.

\begin{tabular}{|c|c|c|c|c|c|c|c|c|c|c|c|}
\hline-11 & 1 & 40 & -21 & 2 & 30 & -12 & 2 & 50 & -22 & 1 & 30 \\
\hline \multicolumn{12}{|c|}{ Swapping: } \\
\hline-11 & 2 & 30 & -21 & 1 & 40 & -12 & 2 & 50 & -22 & 1 & 30 \\
\hline \multicolumn{12}{|c|}{ Moving: } \\
\hline-11 & -21 & 1 & 40 & 2 & 30 & -12 & 2 & 50 & -22 & 1 & 30 \\
\hline \multicolumn{12}{|c|}{ Changing the number of cycles: } \\
\hline-11 & 1 & 25 & -21 & 2 & 30 & -12 & 2 & 50 & -22 & 1 & 45 \\
\hline
\end{tabular}

Figure 5.4: Examples of mutation operations.

solution as soon as possible. In each generation we perform either one of the following operations with the same probability (depicted in Fig. 5.4):

- Swapping: $i$ and $t$, together with their corresponding number of cycles, exchange their positions in the solution. However, in order to avoid creating solutions which are not viable, $i$ and $t$ have to belong to the cores that are executed in parallel (defined with consecutive allocation codes that are in the same $(V, f)$ state). In Fig. 5.4 we can observe that tasks 1 and 2 are swapped between cores 1 and 2 , and both cores are in state 1 .

- Moving: move $i$ to a random position $j$. For the same reason as before, the position $j$ has to belong to a core being executed in parallel as $i$ 's. In Fig. 5.4 we can observe that the first part of task 1 ( 40 cycles) is moved to core 2 , before task 2.

- Changing the number of cycles: assigns a different number of cycles to all the appearances of task $i$, in a way the total number of cycles of that task remains the same. In Fig. 5.4 we can observe that task 1 after the change executes 25 cycles on core 1 in state 1 and 45 cycles on core 2 in state 2 . 


\section{Objective Functions}

Execution Time One objective of our optimisation problem is to minimize the total execution time of the schedule, which is the time spent since the first task starts its execution until the last task finishes its execution. However, since the initial population is randomly created, it is possible that some of the tasks miss their deadlines, making the solution unviable. Assuming that these solutions can provide some quality genetic material, we do not want to discard them completely, but we penalize them by adding the amount of time the tasks have missed their deadlines to the objective function. Thus, the time objective function for $n$ cores and $k$ different tasks is the following:

$$
\hat{T}=T+\sum_{1 \leq i \leq n}\left(\sum_{1 \leq j \leq k} x_{i, j} \cdot y_{j} \cdot\left(s_{i, j}+\tau_{i, j}-\text { deadline }_{j}\right)\right)
$$

where $T$ is the total execution time, given by:

$$
T=\max _{\substack{1 \leq i \leq n \\ 1 \leq j \leq k}}\left(x_{i, j} \cdot\left(s_{i, j}+\tau_{i, j}\right)\right)-\min _{\substack{1 \leq i \leq n \\ 1 \leq j \leq k}}\left(x_{i, j} \cdot s_{i, j}\right)
$$

where $s_{i, j} \geq 0$ is the moment when task $j$ is scheduled on core $i\left(s_{i, j}=0\right.$ if the task $j$ is not scheduled on core $i), \tau_{i, j}$ is the execution time of task $j$ on core $i, x_{i, j}$ is a binary value, that represents whether the task $j$ is executed on core $i\left(x_{i, j}=1\right)$ or not $\left(x_{i, j}=0\right)$. The second part of formula (5.2) represents the penalisation, where $y_{j}$ is another binary value that expresses whether the task $j$ has missed its deadline, deadline ${ }_{j},\left(y_{j}=1\right)$ or $\operatorname{not}\left(y_{j}=0\right)$.

Energy Consumption This objective represents the total energy consumption of the given schedule. In the most general case it is given by the following formula:

$$
E=\sum_{1 \leq i \leq n}\left(P_{s t, i} \cdot T+\sum_{1 \leq j \leq k}\left(x_{i, j} \cdot p_{i, j} \cdot \tau_{i, j}\right)\right)
$$

where $P_{s t, i}$ is the static power of core $i, T$ is the same as in formula (5.3), $p_{i, j}$ is the dynamic power of task $j$ when executed on core $i$, and $x_{i, j}$ and $\tau_{i, j}$ are the same as in formula (5.2). In this work we use static analysis to estimate the energy of single tasks, which will be explained in more detail in Section 5.2.1, while the energy is the sum of the energies of all the tasks, as given in formula (5.4).

The Modified YDS Algorithm YDS [102] is a well known algorithm for energyefficient scheduling for single core DVFS-enabled environments. We have chosen it because it always finds a feasible (and optimal) solution that minimises the total energy consumption, it is simple and fast. However, it does not take into account the static power, which nowadays forms an important part of the total power. YDS reduces the frequency and voltage in order to minimise the dynamic power in a way that the execution time of tasks are extended to their deadlines. However, this also results in an increase of the static energy. Thus, there is a critical point from which further reduction of voltage and frequency actually starts increasing the energy consumption. Attempts 
to reducing the static energy when applying DVFS have mainly tried to group the inactive periods by moving task executions towards their deadline or its release point, and turning off the chip during such periods [49]. However, this is not always possible since chip wake up can take more time than available. Our alternative proposal does not turn off the chip, but instead, finds the critical $(V, f)$ point below which further decrease is not beneficial. Thus, our modification of YDS consists of the following steps:

1. In order to decide if it is beneficial to further decrease the frequency, and in this way avoid the problem when the increase in energy consumed by the static power leads to the total energy increase, we use the simple slope-based method presented in [72].

2. In order to support the multicore system, we propose two different heuristics for allocating the tasks to different cores: the load balanced solution and the solution where a task allocation leads to the minimal frequency increase. After the allocation, the YDS algorithm is applied to each core.

3. In order to adapt the algorithm so that it assigns only the frequencies supported by the system, we propose to divide the computational load into two parts and execute them on two supported frequencies in a way the total execution time remains (almost) the same.

A Solution to the Static Power Issue of YDS As mentioned before, we use the simple slope-based method presented in [72]. The only requirement for its application is the availability of a power model where the static and dynamic power are separated. The main idea of the method is the following. If we fix the voltage, the power is a linear function of the frequency, and after applying some simple numeric transformations (explained in [72] in detail), it can be expressed in this way:

$$
P_{f}=P_{f_{\min }}+m \cdot\left(f-f_{\text {min }}\right)
$$

where $P_{f}$ denotes that the power depends on frequency $f$, and $f_{\min }$ is the minimal possible frequency, assumed to be the one which permits the execution of tasks to finish at their deadlines, and $m$ is called the slope of the power function. If we can compare energies at different frequencies, we will know if it is energy efficient to decrease the frequency. Since power is a function of $m$, the same applies to energy, and thus it determines the decision of decreasing the frequency. In theory, there should exist a slope at which energy is equal for all frequencies. This slope is called the critical power slope:

$$
m_{\text {critical }}=\frac{P_{f_{\min }}-P_{\text {idle }}}{f_{\min }}
$$

If the actual slope $m$ (calculated from Eq. 5.5) is greater than the critical one then we can decrease the frequency in order to save energy. However, if the slope is lower 
than the critical one, then the frequency should be increased in order to save the energy. Since the voltage can also change, the slope should be calculated for each $\left(V_{x}, f_{x}\right)$ point for each frequency $f_{x}$ :

$$
m_{c r i t i c a l}^{f_{x}}=\frac{P_{f_{x}}-P_{i d l e}}{f_{x}}
$$

This value is then compared with the actual slope $m^{f_{x}}$ at each $\left(V_{x}, f_{x}\right)$ point with frequency $f_{x}$. Again, if $m^{f_{x}}>m_{\text {critical }}^{f_{x}}$, we should decrease the frequency in order to save the energy. However, if $m^{f_{x}}<m_{\text {critical }}^{f_{x}}$, the frequency should be increased in order to save the energy.

Optimal Task-Core Allocation Other aspects of adapting YDS to a multicore environment consist of finding an optimal number of cores, and allocation, i.e., assignments of tasks to cores. In this work we do not deal with the first part of the problem, we just show that an optimal number of cores exists and it is not necessarily equal to the maximal possible number of cores. Regarding the second part, we have tested two possibilities:

1. Assign tasks to the cores so that the load is equally distributed between them.

2. Assign tasks in the way its addition assumes minimal change in frequency: a task $t$ is assigned to the core with minimal activity, measured as the number of clock cycles in its active period $\left(t \_r e l e a s e \_t i m e, t \_d e a d l i n e\right)$.

Assigning Frequencies Supported by the System If the frequency $f$ calculated by YDS is not supported by the system, the total number of cycles $\omega_{i}$ is divided in two parts, $\omega_{i 1}$ and $\omega_{i 2}$, which are executed on two frequencies $f_{1}$ and $f_{2}\left(f_{1} \leq f \leq f_{2}\right)$ supported by the underlying system. The values of $\omega_{i 1}$ and $\omega_{i 2}$ are calculated by solving the following system of equations:

$$
\begin{aligned}
& \frac{\omega_{i}}{f} \approx \frac{\omega_{i 1}}{f_{1}}+\frac{\omega_{i 2}}{f_{2}} \\
& \omega_{i}=\omega_{i 1}+\omega_{i 12}
\end{aligned}
$$

\section{Energy Static Analysis as Input}

In order to estimate the energy consumed by programs without actually running them we use the static analysis described in Chapter 2. The functions inferred by the static analysis are arithmetic functions (polynomial, exponential, logarithmic, etc.) that depend on input data sizes (natural numbers). We use them in our scheduling and allocation algorithm to estimate the energy consumed by the different tasks involved. Such estimation can be computed very efficiently once the input data sizes of the tasks are known, since all the basic arithmetic functions involved can be evaluated in little bounded time. 
Table 5.1: Viable $(V, f)$ pairs for XMOS chips.

\begin{tabular}{|l|l|l|l|l|l|l|}
\hline Voltage $(V)$ & 0.95 & 0.87 & 0.8 & 0.8 & 0.75 & 0.7 \\
frequency $(\mathrm{MHz})$ & 500 & 400 & 300 & 150 & 100 & 50 \\
\hline
\end{tabular}

\subsubsection{Experimental Evaluation}

XMOS Chips In this work we target the XS1-L architecture of the XMOS chips as a proof of concept. Although these chips are multicore and multithreaded, in this work we assume a single core architecture with 8 threads, which is the architecture for which we have an available energy model. In this case, we can use the algorithm and representation of individuals described in Section 5.2.1 by considering that a thread in our experiments is conceptually equivalent to a core executing tasks sequentially, as described previously. We refer the reader to [11] for a description of a representation of individuals whose allocation codes include three digits, representing: a core, a thread running in parallel on that core, and a $(V, f)$ state.

In the XS1-L architecture, the threads enter a 4-stage pipeline, meaning that only one instruction from a different thread is executed at each pipeline stage. If the pipeline is not full, the empty stages are filled with NOPs (no operation). Effectively, this means that we can assume that the threads are running in parallel, with frequency $F / N$, where $F$ is the frequency of the chip, and $N=\max (4$, numberOfThreads). DVFS is implemented at the chip level, which means that all the cores have the same voltage and frequency at the same time. In order to apply DVFS, we need a list of VoltageFrequency $(V, f)$ pairs or ranges that provide a correct chip functioning. We have experimentally concluded that the XMOS chips can function properly with the voltage and frequency levels given in Table 5.1.

Task Set In order to test our proposed approach, we use two different groups of task sets. The first group is made up of small tasks, where the EA training with the programlevel energy model takes around one day to complete. This group is used to show the difference between the results obtained with the EA trained with the programlevel energy model and the EA trained with the energy estimations obtained by the static analysis. In this group, we use four different arithmetic programs: fact $(\mathrm{N})$, for calculating the factorial of $\mathrm{N}$, fibonacci $(\mathrm{N})$ for calculating the Nth Fibonacci number, sqr (N) for computing $N^{2}$ and power_of two (N) for computing $2^{N}$. In total, we have created a set of 22 tasks to be scheduled, corresponding to the execution of the previous programs with different inputs $N$.

In the second group we use real world programs, where the EA training based on the program-level energy model is not practical: $\mathrm{fir}(\mathrm{N})$, i.e., Finite Impulse Response (FIR) filter, which in essence computes the inner-product of two vectors of dimension $\mathrm{N}$, a vector of input samples, and a vector of coefficients, and biquad $(\mathrm{N})$, which is a part of an equaliser implementation, based on a cascade of Biquad filters, whose consumed energy depends on the number of banks N. We have used four different FIR implementations, with different number of coefficients: 85, 97, 109 and 121. Fur- 
Table 5.2: Energy functions inferred by static analysis for 3 different pairs of voltage (V)/frequency $(\mathrm{MHz})$.

\begin{tabular}{|c|c|c|c|}
\hline & $\mathbf{V = 0 . 7 0}$ & $\mathbf{V = 0 . 7 5}$ & $\mathbf{V}=\mathbf{0 . 8 0}$ \\
& $\mathbf{F = 5 0}$ & $\mathbf{F}=\mathbf{1 0 0}$ & $\mathbf{F}=\mathbf{1 5 0}$ \\
\hline fact $(N)$ & $60.5 N+46$ & $35 N+26.7$ & $27 N+20.5$ \\
\hline$f i b(N)$ & $87.19 \times 1.62^{N}+$ & $50.32 \times 1.62^{N}+$ & $38.68 \times 1.62^{N}+$ \\
& $26.7 \times(-0.62)^{N}-74.7$ & $15.44 \times(-0.62)^{N}-43$ & $11.85 \times(-0.62)^{N}-33.2$ \\
\hline $\operatorname{sqr}(N)$ & $21.3 N^{2}+121 N$ & $12.3 N^{2}+69.8 N$ & $9.48 N^{2}+53.7 N$ \\
& +39.1 & +22.5 & +17.3 \\
\hline powerOf $(N)$ & $55.1 \times 2^{N}-39$ & $63.7 \times 2^{N}-39$ & $24.49 \times 2^{N}-30$ \\
\hline \hline $\operatorname{fir}(N)$ & $74.93 N+124.5$ & $43.36 N+71.9$ & $33.41 N+55.2$ \\
\hline $\operatorname{biquad}(N)$ & $386 N+128$ & $223.6 N+74.2$ & $172.5 N+57.2$ \\
\hline
\end{tabular}

thermore, we have used four implementations of the biquad benchmark, with different number of banks: 5, 7, 10 and 14. We have tested our approach in scenarios of 16 and 32 tasks, each one corresponding to such implementations. The tasks corresponding to the same implementation have different release times and deadlines.

The energy consumed by the programs is inferred at compile time by the static analysis described in Section 5.2.1. Such energy is expressed as a function of a parameter $N$, the size of the input, which is only known at runtime. Such functions are given in Table 5.2 for 3 of the 6 different voltage and frequency levels used in this work (for conciseness, as the functions for each program have the same complexity order, but different coefficients). The static analysis assumes that a single program (task) is running on one thread on the XMOS chip, while all other threads are inactive. In this implementation, the EA algorithm approximates the total energy of a schedule by adding the energies of all the tasks. Although in this way we loose precision, the estimation still provides precise enough information for the EA to decide which schedule is better.

Testing Scenarios In our current implementation, we assume no dependency between the tasks since it is not supported by the available energy models. The release times and deadlines of the different tasks are set in different scenarios in order to experimentally show the benefits of DVFS and optimal scheduling, where all the tasks have different release times and deadlines, with tighter deadlines; and that it is important to take into account the static power, especially in the case of loose deadlines.

Scenario 1: Tasks with Loose Deadlines In this scenario the release time of a task $k$, denoted $T_{r e l}^{k}$ is a random moment between 0 and the total execution time at the maximal frequency of all the tasks executed sequentially on a single core. Also, the deadline of a task is a random moment between $T_{r e l}^{k}+10 \times T_{\operatorname{maxf}}^{k}$ and $T_{r e l}^{k}+20 \times T_{\max f}^{k}$, where $T_{\operatorname{maxf}}^{k}$ denotes the execution time of the task at maximum frequency. This way we achieve a scenario with loose deadlines even at a smaller frequency. 
Table 5.3: Energy savings obtained by the modified YDS vs. the original YDS (\%).

\begin{tabular}{|c|c|c|c|c|}
\hline & \multicolumn{2}{|c|}{ Tight deadlines } & \multicolumn{2}{c|}{ Loose deadlines } \\
\hline \hline \#Cores & Alloc. 1 & Alloc. 2 & Alloc. 1 & Alloc. 2 \\
\hline \hline 1 & 4.18 & 4.18 & 6.21 & 6.21 \\
2 & 1.5 & 4.26 & 14.67 & 14.67 \\
3 & -5.26 & 3.17 & 14.67 & 14.67 \\
4 & 2.22 & 2.77 & 8.8 & 8.8 \\
5 & -3.28 & 3.47 & 11.18 & 11.18 \\
6 & 0.95 & 4.34 & 11.82 & 11.82 \\
7 & 4.8 & 3.03 & 10.9 & 10.9 \\
8 & 19.36 & 5.61 & 10.56 & 10.56 \\
\hline
\end{tabular}

Scenario 2: Tasks with Tight Deadlines Here, the release time is the same as in Scenario 1. However, the deadline of a task is a random moment between $T_{r e l}^{k}+5 \times$ $T_{\operatorname{maxf}}^{k}$ and $T_{\text {rel }}^{k}+7 \times T_{\operatorname{maxf}}^{k}$. This way we get tighter deadlines, but also provide a set of tasks which are schedulable on the given platform. Note that the deadlines become even tighter as the frequency decreases.

Results: The Improved YDS Table 5.3 shows the savings of our improved YDS algorithm presented in Section 5.2.1 compared to the original YDS, for different number of cores and for two different ways of task allocation: Alloc. 1, where the load is evenly distributed between the cores, and Alloc. 2, where the addition of a task implies a minimal increase in the frequency. The energy saving resulting from a particular scheduling is calculated using the following formula:

$$
\frac{Y D S \_ \text {original }-Y D S \_ \text {modified }}{Y D S \_ \text {original }} \cdot 100
$$

In Table 5.3, energy savings are achieved in all, but in two cases with tight deadlines. A possible reason could be the fact that all the threads need to have the same frequency always, which means that the maximal necessary frequency is assigned, which is not necessarily be optimal for all the threads. However, in the case of loose deadlines, the savings are much more significant, since the static power plays a more important role.

Results: EA vs. improved YDS Both EA and YDS are implemented in C++. EA extends the MOGAlib library [30] for multiobjective genetic algorithms. In the EA, the population of 200 individuals is evolved for 150 generations. The probability of both crossover and mutation is 0.9 . The mutation is assigned higher probability than usual due to its important role for reaching a viable solution. Since the result of the optimisation process is a set of possible solutions which form the approximated Pareto front, we take the solution with minimal energy consumption where all task deadlines are met.

Table 5.4 presents results comparing the EA trained with the energy estimations provided by static analysis, versus the improved YDS algorithm presented in Sec- 
tion 5.2.1. In the first column, the energy of the final solution calculated by using the program-level energy model is given $\left(E A_{s}\right)$. The second column gives the energy of the final scheduling obtained by the modified YDS algorithm (referred as $Y D S_{m}$ ) using the program-level energy model, while the third column gives the energy saving of the EA trained with static analysis compared to YDS. Finally, the last column shows the energy saving obtained with the EA trained with the program-level energy $\operatorname{model}\left(E A_{m}\right)$ [10], which is only applicable in the scenarios with a small number of numeric tasks. Each row shows statistics for each scenario taken from 10-20 runs of the algorithm for the same scenario, where CI0.01 and CI0.05 represent $99 \%$ and $95 \%$ confidence intervals, meaning that we can claim with $99(95) \%$ certainty that the final result will fall in these intervals.

In order to perform the comparison between the EA and $Y D S_{m}$, in the case of EA and tight deadlines, we present the results when the EA can find a viable solution. However, the EA does not always provide a viable solution in all the scenarios with tight deadlines created as explained previously. As we can see in Table 5.4, if the EA finds a viable solution, it always performs better than the $Y D S_{m}$. We can also observe that the EA trained with the program-level energy model achieves better results. However, the EA trained with the energy estimations by static analysis still achieves very good results, but with the training process that lasts around 10 minutes, compared to around 24 hours of training the EA with the program-level energy model, which makes it much more practical.

\subsubsection{Section Conclusions and Future Work}

In this section we propose a holistic approach for optimal scheduling, allocation and $\operatorname{voltage}(V)$ and frequency $(f)$ assignment in multicore environments, adapted for multicore XMOS chips. The main part of our approach is based on our custom developed EA-algorithm, which relies on static analysis to efficiently estimate the energy of input tasks. The use of such static analysis improves significantly the speed of the EA training process, thus allowing its real world applicability. Furthermore, since in the case of very tight task deadlines the EA can fail in providing a feasible solution, we add one more stage based on the YDS algorithm, which has been adapted for multicore environments and improved in a way it takes into account the static power. In this way, we have developed an efficient approach which is capable of providing energy savings in each possible scenario.

However, although the use of static analysis based estimations still provides energy savings, we have seen that better results can be achieved with more precise energy estimations. For this reason, we plan to use a static analysis of the energy consumed by concurrent programs, which is expected to provide additional savings. As a future work, we also plan to study the effect of different versions of the crossover and mutation operators in different situations, which could enable adding a heuristic for choosing the optimal version for each possible scenario. 
Table 5.4: EA vs. improved YDS in different scenarios.

\begin{tabular}{|c|c|c|c|c|}
\hline & $E A_{s}(\mu J)$ & $Y D S_{m}(\mu J)$ & $\frac{\left(Y D S_{m}-E A_{s}\right)}{Y D S_{m}}(\%)$ & $\frac{\left(Y D S_{m}-E A_{m}\right)}{Y D S_{m}}(\%)$ \\
\hline \multicolumn{5}{|c|}{ A scenario with 22 small numeric tasks and loose deadlines } \\
\hline Mean & 14.3 & 33.1 & 56.8 & 76.57 \\
CI 0.01 & $11.6-17$ & NA & $48.64-64.95$ & $67.87-85.27$ \\
CI 0.05 & $12.2-16.4$ & NA & $50.45-63.14$ & $70.05-83.09$ \\
\hline \multicolumn{5}{|c|}{ A scenario with 22 small numeric tasks and tight deadlines } \\
\hline Mean & 14.6 & 34.8 & 60.92 & 69.83 \\
CI 0.01 & $11.5-17.7$ & NA & $49.14-66.95$ & $57.18-57.18$ \\
CI 0.05 & $12.2-17$ & NA & $51.15-64.94$ & $60.34-60.34$ \\
\hline A scenario with 16 tasks made of Biquad and FIR filters and loose deadlines \\
\hline Mean & 4.38 & 35.3 & 87.59 & NA \\
CI 0.01 & $3.4-5.3$ & NA & $85-90.37$ & NA \\
CI 0.05 & $3.7-5.1$ & NA & $85.55-89.52$ & NA \\
\hline \multicolumn{7}{|c|}{ A scenario with 16 tasks made of Biquad and FIR and tight deadlines } \\
\hline Mean & 14.5 & 35.4 & 59.04 & NA \\
CI 0.01 & $9.4-19.6$ & NA & $44.63-73.45$ & NA \\
CI 0.05 & $10.6-18.4$ & NA & $48.02-70.06$ & NA \\
\hline A scenario with 32 tasks made of Biquad and FIR filters and loose deadlines \\
\hline Mean & 17.85 & 68.16 & 73.81 & NA \\
CI 0.01 & $10.8-25$ & NA & $63.32-84.15$ & NA \\
CI 0.05 & $12.5-23.3$ & NA & $65.82-81.66$ & NA \\
\hline A scenario with 32 tasks made of Biquad and FIR filters and tight deadlines \\
\hline Mean & 29.43 & 68.16 & 56.82 & NA \\
CI 0.01 & $0.72-51.6$ & NA & $24.3-89.44$ & NA \\
CI 0.05 & $12.5-46.3$ & NA & $32.07-81.66$ & NA \\
\hline
\end{tabular}




\subsection{Trade-off Accuracy vs. Energy for Energy Efficient Scheduling}

In this section we want to deal with optimally scheduling tasks which can permit certain accuracy loss. As a matter of fact, the great majority of today's processors are designed in a way that can provide a high level of accuracy. However, there are numerous applications that allow certain accuracy loss, which still permits them to function properly, such as video streaming, machine learning, etc. Since decreasing the accuracy is usually achieved by reducing the computational load, this can lead to both increase in performance and decrease in energy consumption, so here we deal with a trade-off between accuracy on one side and performance and/or energy on the other. One technique that achieves this is loop perforation [89], which in essence consists in skipping every $n$-th loop iteration, for a given $n$. Broadly speaking, accuracy can be considered as one aspect of quality of service (QoS), so we can say that in this work we deal with the QoS/energy trade-off.

Thus, in this work we solve the following scheduling problem: given a set of tasks with known release time and number of cycles to compute them, find proper allocation and scheduling of the tasks, as well as a $(V, f)$ assignment (i.e., voltage and frequency pair) to the cores in a way the total energy is minimised, while accuracy is maximised, meeting a minimal acceptable level of accuracy. Different levels of accuracy are achieved by applying the loop perforation technique with different $n$, where every $n$-th loop iteration is skipped.

Hence, we deal with two objectives: accuracy and energy. Accuracy is defined in terms of deviations of the output signal after applying the loop perforation, while in order to estimate energy consumption, we use an existing static analysis which, at compile time, with no need of executing the programs, and in a matter of seconds, gives a safe estimation of the energy consumed by programs. The energy consumption often depends on (the size of) input data, which is not known at compile time. For this reason, the static analysis provides the energy as a function of the input parameters, which is evaluated when input values are known at runtime. The energy consumption estimated by using the static analysis for a given scheduling is calculated as the sum of energies of the tasks running on different cores. This gives a safe upper bound on the total energy consumption, which is good enough for deciding which schedule consumes less energy, and can provide acceptable estimations of energy savings.

The rest of the section is organised as follows. Section 5.3.1 gives more details of our proposed approach. Section 5.3.2 presents an experimental evaluation of it. Some related work is discussed in Section 5.3.3 and finally, some conclusions are drawn in Section 5.3.4. 


\begin{tabular}{|c|c|c|c|c|c|c|c|c|c|}
\hline$\ldots$ & -12 & 1 & 48 & 0 & 5 & 77 & 4 & -24 & $\ldots$ \\
\hline & $\begin{array}{l}\text { Core } 1 \\
\text { state } 2\end{array}$ & Task 1 & $\begin{array}{c}48 \\
\text { cycles } \\
\text { of task } 1\end{array}$ & $\begin{array}{l}\text { No loop } \\
\text { perforation }\end{array}$ & Task 5 & $\begin{array}{c}77 \\
\text { cycles } \\
\text { of task } 5\end{array}$ & $\begin{array}{l}\text { Take out } \\
\text { every } 4 \text { th } \\
\text { iteration }\end{array}$ & $\begin{array}{l}\text { Core 2, } \\
\text { state } 4\end{array}$ & \\
\hline
\end{tabular}

Figure 5.5: Representation of an individual

\subsubsection{Proposed Approach}

Loop Perforation. The loop perforation technique consists in skipping some loop iterations, for example skipping every $n$-th iteration [89], where $n$ can be varied in order to trade accuracy with energy, i.e., for higher $n$, less instructions are skipped, so the accuracy is higher, while more energy is saved for lower values of $n$. This tradeoff between accuracy and energy consumption justifies the usage of a multiobjective algorithm. As we will see in the following, in this work the loop perforation technique is implemented as one possibility for the mutation operator.

Evolutionary Algorithm (EA). The work presented in this section is an extension of our previous work where we developed a custom algorithm based on an NSGA-II multiobjective evolutionary algorithm [11]. The conflicting objectives are accuracy and energy consumption, since we want to decrease the energy consumption, while maintaining the accuracy level as high as possible (always above a given threshold).

The non-dominated solutions are generated using the well-known NSGA-II algorithm [23], while the EA follows the standard steps of evolutionary algorithms: initialisation, evolution, where the selection process is implemented as standard tournament selection, and our custom-made crossover and mutation operators are applied. In the following we give more detail on the particular improvements carried out in this work.

Individual. A solution to the problem we are solving has to contain information about scheduling and allocation of each task, how many cycles of each task are executed in the current run (since we support task migration), and voltage and frequency levels of the core at each moment. In this work we add a new dimension to the problem, which is the possibility to decrease accuracy through loop perforation and thus it also has to be encoded in the individual. For this reason, we add one more field after each task, which encodes $n$, i.e., the iterations which can be skipped in one or more loops previously identified in each task. An example of a part of an individual is given in Figure 5.5, and can be read in the following way: on core 1 in state 2 we execute in this order,

- 48 cycles of task 1 , without performing loop perforation on it, and

- 77 cycles of task 5, where we skip every 4th iteration in the loop previously defined.

Population Initialisation. Individuals in the initial population are created by randomly assigning tasks to random cores in random $(V, f)$ settings with equal probability. However, in order to provide a load balanced solution (as much as possible), the probability 
of choosing a core decreases as its load increases. The number of cycles of a task executed in each run, as well as the loop iterations to be skipped are also randomly chosen.

The Crossover Operator. Our custom crossover operator is designed in the following way:

- Each child preserves the order of appearance of the tasks, as well as their allocation from one of the parents,

- But, can take the distribution of the number of cycles, as well as the number of loop iterations to be skipped of one of them with equal probability.

The Mutation Operator. The mutation operator can perform different operations involving one or two tasks (designated as $i$ and $t$ in the following text). In each generation we perform one of the following operations with the same probability:

- Swapping: $i$ and $t$, together with their corresponding number of cycles and loop iterations to be skipped, change their positions in the solution. However, in order to avoid creating solutions which are not viable, $i$ and $t$ have to belong to the cores which are executed in parallel.

- Moving: move $i$ to a random position $j$. For the same reason as before, the position $j$ has to belong to a core being executed in the same state as $i$ 's original state.

- Changing the cycle distribution: Randomly change distribution of the cycles of task $i$ between its appearances on different cores.

- Loop Perforation: For a random task $i$, assign randomly the number of loop iterations to be skipped, update the total number of cycles, i.e., decrease the total number of cycles for the amount corresponding to the cycles of the skipped loops, and share them randomly between the existing appearances of the task $i$ in the solution.

These operators are depicted in Fig.5.6:

- Swapping: Tasks 1 and 2 are swapped between cores 1 and 2 while both in state 1.

- Moving: First part of task 1 (40 cycles) are moved to core 2 before task 2.

- Changing the number of cycles: Task 1 now executes 25 cycles on core 1 in state 1 and 45 cycles on core 2 and state 2 .

- Loop perforation: Task 1, where loop perforation has not been performed, now skips every 20th task in the defined loop, which results in decreased number of cycles, i.e., it has 60 cycles, where the first 35 cycles are executed in the first appearance of the task 1 , while the remaining 25 cycles are executed in its second appearance. 


\begin{tabular}{|c|c|c|c|c|c|c|c|c|c|c|c|c|c|c|c|}
\hline-11 & 1 & 40 & 0 & -21 & 2 & 30 & 10 & -12 & 2 & 50 & 10 & -22 & 1 & 30 & 0 \\
\hline \multicolumn{16}{|c|}{ Swapping: } \\
\hline-11 & 2 & 30 & 10 & -21 & 1 & 40 & 0 & -12 & 2 & 50 & 10 & -22 & 1 & 30 & 0 \\
\hline \multicolumn{16}{|c|}{ Moving: } \\
\hline-11 & -21 & 1 & 40 & 0 & 2 & 30 & 10 & -12 & 2 & 50 & 10 & -22 & 1 & 30 & 0 \\
\hline \multicolumn{16}{|c|}{ Changing the number of cycles: } \\
\hline-11 & 1 & 25 & 0 & -21 & 2 & 30 & 10 & -12 & 2 & 50 & 10 & -22 & 1 & 45 & 0 \\
\hline \multicolumn{16}{|c|}{ Loop perforation: } \\
\hline-11 & 1 & 35 & 20 & -21 & 2 & 30 & 10 & -12 & 2 & 50 & 10 & -22 & 1 & 25 & 20 \\
\hline
\end{tabular}

Figure 5.6: Different possibilities for mutation

Objective Functions: Energy Consumption. This objective represents the total energy consumption of the given schedule, and it should be minimised. It is given with the following formula:

$$
E=\sum_{1 \leq i \leq n}\left(P_{s t, i} \cdot T+\sum_{1 \leq j \leq k}\left(x_{i, j} \cdot p_{i, j} \cdot \tau_{i, j}\right)\right)
$$

where $P_{s t, i}$ is the static power of the core $i, T$ is the total execution time of the schedule, i.e., the moment when the last task finishes its execution, $\tau_{i, j}$ is the execution time of task $j$ on core $i, x_{i, j}$ is a binary value, $x_{i, j} \in\{0,1\}$, that represents whether the task $j$ is executed on the core $i\left(x_{i, j}=1\right)$ or not $\left(x_{i, j}=0\right)$, and $p_{i, j}$ is the power of task $j$ when executed on core $i$.

Objective Functions: Accuracy. In this work accuracy is defined as an average error of the output after applying loop perforation, and it should be minimised. If a task performs some sort of signal processing, where the output is a digital signal consisting of a number of samples, the error is calculated as the Euclidean distance between the outputs obtained with and without loop perforation.

Energy Static Analysis as Input. As described in Section 5.2, in order to statically estimate the energy consumed by programs we use the analysis framework described in Chapter 2.

\subsubsection{Experimental Evaluation}

\section{Testing Environment}

XMOS Chips. In this work we target the XS1-L architecture of the XMOS chips as a proof of concept. Although these chips are multicore and multithreaded, in this work we assume a single core architecture with 8 threads, which is the architecture for which we have an available energy model. All threads have their own register set 
Table 5.5: Viable $(V, f)$ pairs for XMOS chips.

\begin{tabular}{|l|l|l|l|l|l|l|}
\hline Voltage $(\mathrm{V})$ & 0.95 & 0.87 & 0.8 & 0.8 & 0.75 & 0.7 \\
\hline frequency $(\mathrm{MHz})$ & 500 & 400 & 300 & 150 & 100 & 50 \\
\hline
\end{tabular}

and up to 4 instructions per thread can be buffered, which are scheduled in a way to minimize simultaneous memory accesses by consecutive threads. The threads enter a 4-stage pipeline, meaning that only one instruction from a different thread is executed at each pipeline stage. If the pipeline is not full, the empty stages are filled with NOPS (no operation). Effectively, this means that we can assume that the threads are running in parallel, with frequency $F / N$, where $F$ is the frequency of the chip, and $N=\max (4$, numberOfThreads $)$.

DVFS is implemented at the chip level, which means that all the threads have the same voltage and frequency at the same time. All XMOS chips support frequency scaling. However, only the XS1-SU01A-FB96 [100] chip provides the possibility of voltage scaling enabled by two DC-DC converters whose output voltage belongs to the range $(0.6 \mathrm{~V}, 1.3 \mathrm{~V})$. In order to apply DVFS, we need list of Voltage-Frequency $(V, f)$ pairs or ranges that provide a correct chip functioning. We have experimentally concluded that the XMOS chips can function properly with the voltage and frequency levels given in Table 5.5.

Task Set. We use two real world programs for testing:

- $\operatorname{fir}(\mathrm{N})$ : Finite Impulse Response (FIR) filter. In essence, it computes the innerproduct of two vectors: a vector of input samples, and a vector of coefficients.

- biquad (N): Part of an equaliser implementation, which uses a cascade of Biquad filters. The energy consumed depends on the number of filters in the cascade, also known as banks $\mathrm{N}$.

These filters are often used in signal processing, where some certain level of accuracy loss can be permitted. This makes them good candidates for experimenting with the accuracy/energy trade-off. We have used four different FIR implementations, with different number of coefficients: 85, 97, 109 and 121. Furthermore, we have used four implementations of the biquad program, with different number of banks: 5, 7, 10 and 14. We have tested our approach in scenarios with 32 tasks, each one corresponding to one of the above mentioned implementations. The tasks corresponding to the same implementation have different release times.

The energy consumed by the programs is inferred at compile time by the static analysis described in Chapter 2. This energy is expressed as a function of an input parameter $N$, which is known at run time only. In the case of FIR, $N$ is the number of coefficients, while in the case of the Biquad cascade, $N$ is the number of banks. These functions are given in Table 5.6. The analysis assumes that a single program is running on one thread on the XMOS chip, while all other threads are inactive. This means that only the first stage of the pipeline is occupied with an instruction, while the 
Table 5.6: Energy functions for 3 different pairs of voltage (V) / frequency (F, in MHz)

\begin{tabular}{|c|c|c|c|}
\hline & $\mathbf{V}=\mathbf{0 . 7 0}$ & $\mathbf{V}=\mathbf{0 . 7 5}$ & $\mathbf{V}=\mathbf{0 . 8 0}$ \\
& $\mathbf{F}=\mathbf{5 0}$ & $\mathbf{F}=\mathbf{1 0 0}$ & $\mathbf{F}=\mathbf{1 5 0}$ \\
\hline fir $(N)$ & $74.93 N+124.5$ & $43.36 N+71.9$ & $33.41 N+55.2$ \\
\hline $\operatorname{biquad}(N)$ & $386 N+128$ & $223.6 N+74.2$ & $172.5 N+57.2$ \\
\hline & $\mathbf{V}=\mathbf{0 . 8 0}$ & $\mathbf{V}=\mathbf{0 . 8 7}$ & $\mathbf{V}=\mathbf{0 . 9 5}$ \\
& $\mathbf{F}=\mathbf{3 0 0}$ & $\mathbf{F}=\mathbf{4 0 0}$ & $\mathbf{F}=\mathbf{5 0 0}$ \\
\hline $\operatorname{fir}(N)$ & $20.14 N+33.2$ & $18.95 N+31.09$ & $19.15 N+31.3$ \\
\hline $\operatorname{biquad}(N)$ & $104.3 N+34.4$ & $98.31 N+32.4$ & $99.48 N+32.7$ \\
\hline
\end{tabular}

rest are empty, i.e., occupied with NOPs. In this implementation, the EA algorithm approximates the total energy of a schedule taking the sum of the energies of all the tasks running on different cores, i.e., threads, as we have seen in Section 5.3.1. However, in reality if all the threads are active and execute a program, each pipeline stage will contain an instruction from a different thread. For this reason, we can say that the estimation produced by the static analysis of the energy consumed by a set of tasks is an upper bound on the actual energy consumption. However, this estimation provides precise enough information for the EA to decide which schedule is better.

\section{Testing Scenario}

We have tested our approach on a scenario of 32 tasks, where each task implements either an FIR or a Biquad cascade previously described. For the case of FIR, loop perforation takes out a few coefficients, while in the case of Biquad cascade, it takes out a few banks. All tasks have different release time. Task deadlines do not exist. However, we should bear in mind that in the case of DVFS it is not beneficial to scale down voltage and frequency indefinitely, since at some point static power consumption becomes more significant than dynamic power consumption. Thus, if we keep decreasing the dynamic power, the static power is increased at the same time, and as a result, the total energy consumption increases. The input signal to all tasks is a standardised set of input samples for testing in signal processing.

\section{Obtained Results and Discussion}

The EA has been trained with the following parameters: population of 200 individuals, evolved for 150 generations, crossover rate: 0.9 , and mutation rate: 0.9 - since mutation introduces loop perforation, a high rate is needed.

In order to illustrate the energy savings provided by loop perforation (referred to as Case 1 in the following text), we have trained another EA, where the objectives are to minimize energy and execution time, without the possibility of loop perforation (referred to as Case 2 in the following). This algorithm has been trained with the same parameters given above. Since both algorithms are multiobjective, the result of the training of both is a Pareto front of possible solutions with different trade-off between the objectives. Examples of Pareto fronts obtained in Case 1 and Case 2 are given in Figures 5.7 and 5.8 respectively. In Case 1 we have picked a solution with the smallest energy objective value, whose maximal deviation from the final result (accuracy) is 


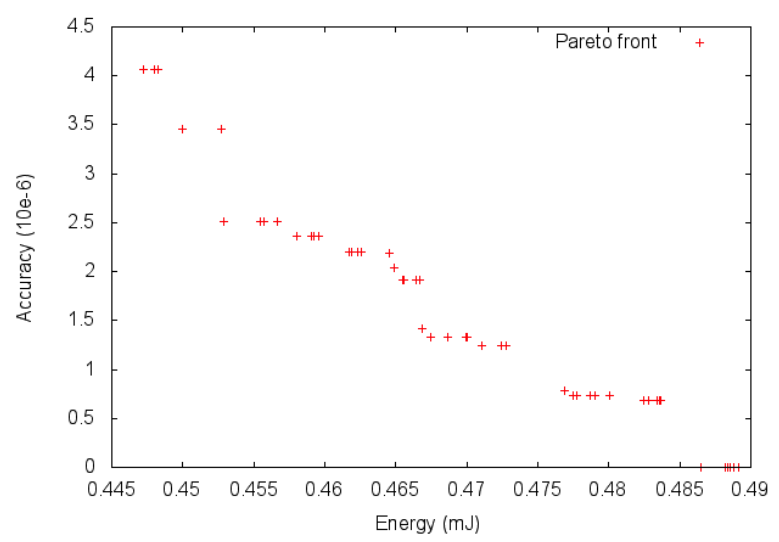

Figure 5.7: Pareto front for Energy/Accuracy trade-off EA (Case1)

Table 5.7: Obtained savings with different levels of minimal acceptable accuracy.

\begin{tabular}{|c|c|c|c|c|}
\hline Max. & Case 1: & Case 2: & \multicolumn{2}{|c|}{ Savings(\%) } \\
\cline { 4 - 5 } Avg. Error & Avg. En.(mJ) & Avg. En.(mJ) & Avg. & CI0.05 \\
\hline $10^{-6}$ & 0.487 & 0.721 & 16.18 & $0.93-31.42$ \\
\hline $2 \cdot 10^{-6}$ & 0.461 & 0.597 & 18.21 & $3.54-32.87$ \\
\hline $3 \cdot 10^{-6}$ & 0.434 & 0.666 & 31.04 & $13.72-48.37$ \\
\hline
\end{tabular}

below (above) a given threshold, while in Case 2 we have chosen a solution with the smallest energy objective. The results are presented in Table 5.7, with the following columns:

- Column 1: Maximal acceptable average error (or equivalently, minimal acceptable level of accuracy) of the final result.

- Column 2: Average energy of the final schedule obtained in a set of experiments of Case 1 estimated by static analysis given in $m J$ (mili Joules).

- Column 3: Average energy of the final schedule obtained in a set of experiments of Case 2 estimated by static analysis given in $m J$ (mili Joules).

- Column 4: Obtained savings expressed as \% and calculated as $\frac{\text { Column } 3-\text { Column } 2}{\text { Column } 3} \cdot 100$.

- Column 5: Statistics of the experiments expressed as 0.05 confidence interval, i.e., we can claim with $95 \%$ certainty that the final result will belong to this interval.

As we can observe, energy savings that can be obtained with loop perforation are significant and range from 3\% to $40 \%$ in different experiments, even with small permitted level of error. As we increase the accepted level of average error, the savings increase, as expected, which is clearly depicted in Figure 5.9. However, the relationship 


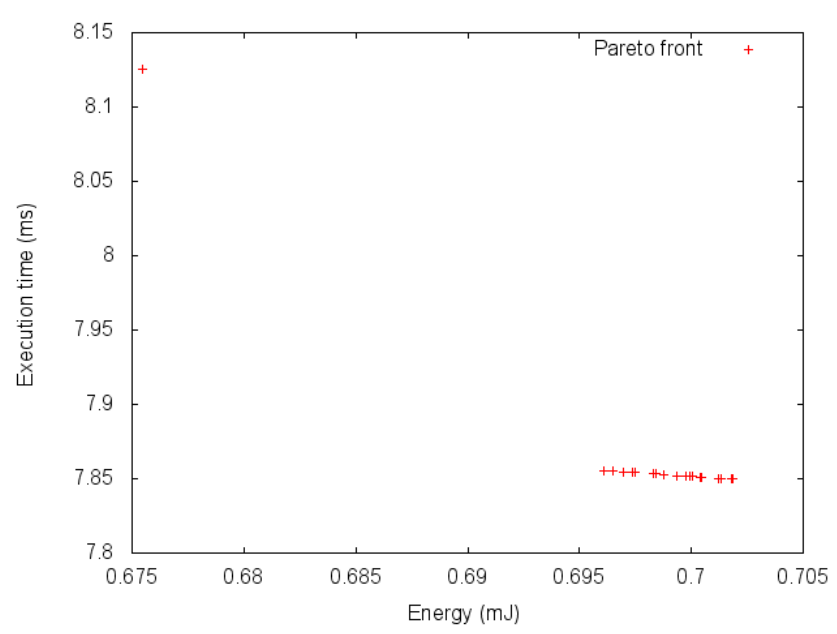

Figure 5.8: Pareto front for Energy/Time trade-off EA (Case 2)

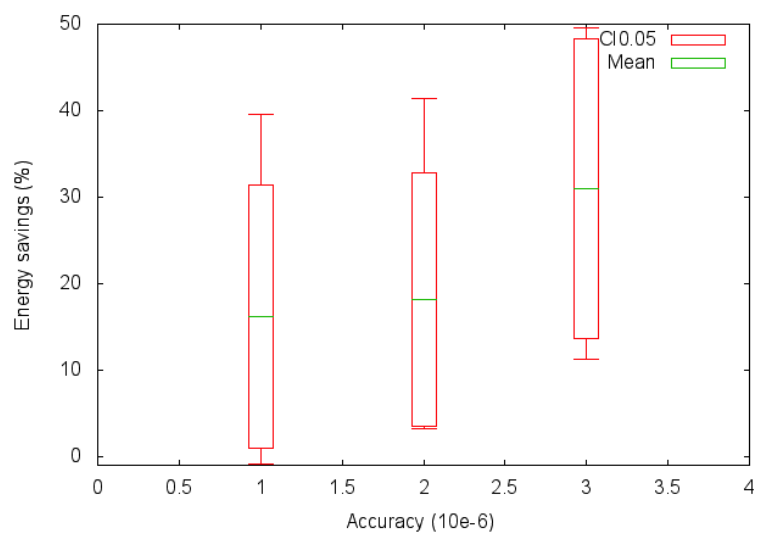

Figure 5.9: Energy savings for different accuracy levels

between the accuracy and the energy savings depends on the application: some applications can preserve acceptable accuracy by skipping more loop iterations (and hence achieve bigger energy savings) than others that lose acceptable accuracy by skipping less loop iterations (and hence achieve smaller energy savings).

Fluctuations in the final result in different experiments appear due to the imprecision of the static analysis, since currently it gives an upper bound, rather than a realistic estimation of energy consumption. This can explain the big confidence intervals. Since the acceptable level of error is small, we could observe that in the final result only tasks that perform FIR could skip a few iterations, while some of the tasks that perform biquad could skip one iteration at most, since the number of iterations is bigger in FIR than in the case of the biquad cascade. In Table 5.8 we present an example of a part of an output containing tasks where loop perforation was applied, where the maximal acceptable error is $10^{-6}$. In the table, for each task, we show the original number of loop iterations, the number of loop iterations after applying loop perforation, and $N$, 
Table 5.8: Result of an experiment: tasks whose final number of loop iterations has been changed.

\begin{tabular}{|c|c|c|c|}
\hline Task & $\begin{array}{c}\text { Original num. of } \\
\text { loop iterations }\end{array}$ & $\begin{array}{c}\text { Final num. of } \\
\text { loop iterations }\end{array}$ & N \\
\hline FIR97-1 & 97 & 87 & 9 \\
\hline FIR85-1 & 85 & 76 & 9 \\
\hline FIR121-1 & 121 & 108 & 9 \\
\hline FIR109-1 & 109 & 104 & 21 \\
\hline FIR97-2 & 97 & 96 & 96 \\
\hline FIR85-2 & 85 & 84 & 84 \\
\hline FIR121-2 & 121 & 120 & 120 \\
\hline FIR109-2 & 109 & 108 & 108 \\
\hline FIR97-3 & 97 & 87 & 9 \\
\hline FIR85-3 & 85 & 76 & 9 \\
\hline FIR121-3 & 121 & 108 & 9 \\
\hline FIR109-3 & 109 & 97 & 9 \\
\hline FIR85-4 & 85 & 84 & 1 \\
\hline FIR121-3 & 121 & 81 & 3 \\
\hline FIR109-3 & 109 & 97 & 9 \\
\hline
\end{tabular}

where every $N$-th loop is skipped. The actual error of this example is $7.8 \cdot 10^{-7}$, but we still achieve significant energy savings.

\subsubsection{Related Work}

In the existing literature techniques that include QoS as an objective in scheduling are mainly designed for Grid or Cloud Computing environments, where QoS is measured as either execution time, cost, etc., which has to be provided according to the signed Service Level Agreement (SLA) between the provider and the customer [104, 103, 105]. Multiobjective genetic algorithms were used in [105] to minimize cost and execution time, since they can be in conflict. A similar approach is presented in [104]. However, in the recent past, energy consumption has become a bottleneck, so it has become very important to reduce it. One such work is given in [103], where the authors try to minimize energy and maximize QoS at the same time in a Cloud Computing environment. The multiobjective optimisation problem is solved using particle swarm optimisation.

However, as far as we know, none of the approaches in the literature propose to trade off QoS (accuracy in our case) with energy or performance in a scheduling problem by transforming the code, in our case by using loop perforation. 


\subsubsection{Section Conclusions}

In this section we have presented an approach for energy efficient scheduling in multicore environments, adapted to multicore XMOS processors, where significant additional energy can be saved if a certain level of accuracy reduction in final result is allowed. Accuracy reduction is performed by using the loop perforation technique. Our experimental results show that, even with small acceptable levels of error in the result, significant energy savings can be obtained.

However, the energy estimation of different schedules is based on a static analysis that can only provide an upper bound. Although it is still capable of providing energy savings, better results could be achieved with more precise energy estimations. For this reason, we are developing an energy analysis of concurrent program, which is expected to provide additional savings. 



\section{Conclusions and Future Work}

The thesis addresses the challenge of promoting energy efficiency to a first class software design goal, providing tools and techniques for energy-aware software development. It mainly focuses on the estimation of lower and upper bounds on the energy consumed by software applications, as well as their energy optimization and the verification that such applications meet a given energy budget.

More concretely, the thesis presents a combination of techniques for static program analysis with techniques for modeling hardware platforms. The energy models are used to represent the effect, regarding energy consumption, of running basic software elements (e.g., low-level instructions or blocks) on the hardware. The static analysis propagates such information through code segments, conditionals, loops, recursions, etc., in order to infer the energy consumption of the whole program. A main contribution of the thesis, in Chapter 2, is a multi-language and multi-platform resource consumption analysis that infers both lower- and upper-bound energy functions at two levels, the Instruction Set Architecture (ISA) and the intermediate code (LLVM IR) levels, and reflects it upwards to the higher source code level. This required the development of translations from both ISA and the LLVM IR to an intermediate representation (Horn clauses) on which the analysis operates on. The experimental assessment of such analysis tool provides insights into the trade-off of precision versus analyzability at these levels, and concludes that the LLVM IR level analysis is a good compromise, since 1) LLVM IR is close enough to the source code level to preserve most of the program information needed by the static analysis, and 2) the LLVM IR is close enough to the ISA level to allow the propagation of the ISA energy model up to the LLVM IR level without significant loss of accuracy for the examples studied, hence making the LLVM IR level reasonably powerful and accurate (less than $6.4 \%$ average deviation w.r.t. hardware measurements).

In our resource usage analysis we have focused on the study of the energy consumption due to computation, so that we have not tested programs where storage and networking are important. However, this could potentially be done in future work, by using the static analysis, which already infers bounds on data sizes, and combining such information with appropriate energy models of communication and storage. 
Moreover, it remains to be seen whether the results would carry over to other classes of programs, such as multi-threaded programs and programs where timing is more important.

We have proposed a combined static/dynamic approach for estimating parametric upper and lower bounds on the energy consumption of a program (Chapter 3). The dynamic part, based on an Evolutionary Algorithm (EA), is a best effort approach to approximating the maximum/minimum energy consumption of the basic blocks (with multiple instructions) in the program, which capture inter-instruction dependencies. Moreover, the basic blocks are branchless, which makes the EA approach quite practical and efficient, and the energy values inferred by it are accurate, since no control flow-related variations occur. Our safe static analysis is then used to combine the energy values obtained for the blocks according to the program control flow, and estimate energy consumption bounds of the whole program that depend on input data sizes. In the experiments we performed on a set of benchmarks, the upper and lower bounds obtained were always safe and quite accurate. Such results suggest that our approach offers a good safety/accuracy compromise, estimating bounds that are practical for its application to energy verification and optimization. On average, the experimental evaluation observed $8.5 \%$ over approximated upper and 9.2\% under approximated lower bounds compared to the worst- and best-case hardware measurements. As already said, the EA captures the instruction dependencies within the blocks, however the inter-block dependencies are not captured, which is a possible source of inaccuracy in the block-level energy models. An improvement left for future work is introducing data analysis of the input values to each block in order to capture the inter-block data dependencies. This will also speedup the EA since it will have bounds on the input values that it can explore.

Traditional static resource analyses estimate the total resource usage of a program without executing it with concrete data. The thesis also presents a novel resource analysis in Chapter 4, whose aim is instead the static profiling of accumulated cost, i.e., to discover, for selected parts of the program (named cost centers), an estimate or bound of the resource usage accumulated in each of those centers, which express how the total cost of a call to the main program is distributed among the different cost centers. Such information is much more useful to the software developer than the traditional resource usage functions, as it allows identifying the parts of a program that should be optimized first, because of their greater impact on the total cost of program executions. We also report on our implementation of the proposed technique using the CiaoPP analysis framework and provide some experimental results. The preliminary experimental results with the tool support the usefulness of our approach where precise accumulated upper bound cost functions were inferred for parts of the program for which the standard analysis was not able to infer precise information.

Be it traditional or accumulated cost analysis, the underlined static analysis infers sound bound representations in the form of recurrence equations, sometimes the external solvers it uses are not able to find closed form functions for such equations. This is a limitation in applications where such closed forms are needed. Techniques to ad- 
dress such limitation are an important line for future work. Our static analysis will also benefit from any improvement of the Computer Algebra Systems used for solving recurrence equations.

Different types of optimisations at different levels of the system stack can be performed by taking advantage of the energy estimations provided by the multi-level and multi-language energy consumption analysis already described. The thesis also proposed novel techniques, in Chapter 5, based on Evolutionary Algorithms to improve energy-efficient task allocation and scheduling for DVFS-enabled multicore environments. These techniques are able to deal with task migration and preemption. For applications that allow certain levels of variability in the accuracy of their computations, the trade-off between Quality of Service (QoS) and energy is investigated as well. The main part of our developed approach is based on a custom EA that relies on static analysis to efficiently estimate the energy of input tasks. The use of such static analysis improves significantly the speed of the EA training process, thus allowing its real world applicability. Other optimisations include the use of energy analysis to choose software parameters in order to transform programs to ensure that an energy target is met while minimizing the loss in QoS.

Finally, as an overall conclusion, the thesis suggests that it is feasible to automatize many tasks in energy-aware software development, and helps promoting energy efficiency to a first class software design goal. 



\section{Bibliography}

[1] S. Albers, F. Müller, and S. Schmelzer. Speed scaling on parallel processors. In Proceedings of the Nineteenth Annual ACM Symposium on Parallel Algorithms and Architectures, SPAA '07, pages 289-298, New York, NY, USA, 2007. ACM.

[2] E. Albert, P. Arenas, S. Genaim, I. Herraiz, and G. Puebla. Comparing cost functions in resource analysis. In 1st International Workshop on Foundational and Practical Aspects of Resource Analysis (FOPARA'09), volume 6234 of Lecture Notes in Computer Science, pages 1-17. Springer, 2010.

[3] E. Albert, P. Arenas, S. Genaim, and G. Puebla. Closed-Form Upper Bounds in Static Cost Analysis. Journal of Automated Reasoning, 46(2):161-203, February 2011.

[4] E. Albert, P. Arenas, S. Genaim, G. Puebla, and D. Zanardini. Cost Analysis of Java Bytecode. In R. D. Nicola, editor, 16th European Symposium on Programming, ESOP'07, volume 4421 of Lecture Notes in Computer Science, pages 157-172. Springer, March 2007.

[5] E. Albert, S. Genaim, and A. N. Masud. More Precise yet Widely Applicable Cost Analysis. In 12th Verification, Model Checking, and Abstract Interpretation (VMCAI'11), volume 6538 of Lecture Notes in Computer Science, pages 38-53. Springer Verlag, January 2011.

[6] D. E. Alonso-Blas and S. Genaim. On the Limits of the Classical Approach to Cost Analysis. In A. Miné and D. Schmidt, editors, Static Analysis, 19th International Symposium (SAS 2012), volume 7460 of Lecture Notes in Computer Science, pages 405-421. Springer Berlin Heidelberg, 2012.

[7] Z. Banković, U. Liqat, and P. López-García. A Practical Approach for Energy Efficient Scheduling in Multicore Environments by combining Evolutionary and 
YDS Algorithms with Faster Energy Estimation. In The 11th International Conference on Artificial Intelligence Applications and Innovations (AIAI'15), volume 458 of IFIP Advances in Information and Communication Technology, pages 478-493. Springer, 2015.

[8] Z. Banković, U. Liqat, and P. López-García. Trading-off Accuracy vs. Energy in Multicore Processors via Evolutionary Algorithms Combining Loop Perforation and Static Analysis-based Scheduling. In E. Onieva, I. Santos, E. Osaba, H. Quintián, and E. Corchado, editors, Hybrid Artificial Intelligent Systems (HAIS 2015), volume 9121 of Lecture Notes in Computer Science, pages 690701. Springer International Publishing, 2015.

[9] Z. Banković, U. Liqat, and P. López-García. A General Methodology for Energy-efficient Scheduling in Multicore Environments based on Evolutionary Algorithms. Journal of Multiple-Valued Logic and Soft Computing (JMVLSC), SOCO’15 Special Issue, 2016.

[10] Z. Banković and P. López-García. Energy Efficient Allocation and Scheduling for DVFS-enabled Multicore Environments using a Multiobjective Evolutionary Algorithm. In Genetic and Evolutionary Computation Conference (GECCO 2015), pages 1353-1354. ACM, 2015.

[11] Z. Banković and P. Lopez-Garcia. Stochastic vs. Deterministic Evolutionary Algorithm-based Allocation and Scheduling for XMOS Chips. Neurocomputing, 150:82-89, February 2015.

[12] N. Bjørner, F. Fioravanti, A. Rybalchenko, and V. Senni, editors. Workshop on Horn Clauses for Verification and Synthesis, July 2014. Electronic Proceedings in Theoretical Computer Science.

[13] C. Boogerd and L. Moonen. On the use of data flow analysis in static profiling. In Source Code Analysis and Manipulation, 2008 Eighth IEEE International Working Conference on, pages 79-88, Sept 2008.

[14] F. Bourdoncle. Abstract debugging of higher-order imperative languages. In Programming Languages Design and Implementation'93, pages 46-55, 1993.

[15] F. Brandner, S. Hepp, and A. Jordan. Static profiling of the worst-case in realtime programs. In Proceedings of the 20th International Conference on RealTime and Network Systems, RTNS 2012, pages 101-110, New York, NY, USA, 2012. ACM.

[16] F. Bueno, P. Deransart, W. Drabent, G. Ferrand, M. V. Hermenegildo, J. Maluszynski, and G. Puebla. On the Role of Semantic Approximations in Validation and Diagnosis of Constraint Logic Programs. In Proc. of the 3rd. Int'l Workshop on Automated Debugging-AADEBUG'97, pages 155-170, Linköping, Sweden, May 1997. U. of Linköping Press. 
[17] S. Chakravarty, Z. Zhao, and A. Gerstlauer. Automated, Retargetable Backannotation for Host Compiled Performance and Power Modeling. In Proceedings of the Ninth IEEE/ACM/IFIP International Conference on Hardware/Software Codesign and System Synthesis, CODES+ISSS '13, pages 36:1-36:10, USA, 2013. IEEE Press.

[18] Y. Chang-tian and Y. Jiong. Energy-aware genetic algorithms for task scheduling in cloud computing. In 7th ChinaGrid Annual Conference (CHINAGRID'12), pages 43-48, 2012.

[19] M. Comini, G. Levi, M. C. Meo, and G. Vitiello. Abstract diagnosis. Journal of Logic Programming, 39(1-3):43-93, 1999.

[20] M. Comini, G. Levi, and G. Vitiello. Declarative diagnosis revisited. In 1995 International Logic Programming Symposium, pages 275-287, Portland, Oregon, December 1995. MIT Press, Cambridge, MA.

[21] P. Cousot. Automatic Verification by Abstract Interpretation, Invited Tutorial. In Fourth International Conference on Verification, Model Checking and Abstract Interpretation (VMCAI), number 2575 in LNCS, pages 20-24. Springer, January 2003.

[22] L. M. de Moura and N. Bjørner. Z3: An Efficient SMT Solver. In C. R. Ramakrishnan and J. Rehof, editors, Tools and Algorithms for the Construction and Analysis of Systems, 14th International Conference, TACAS 2008, volume 4963 of Lecture Notes in Computer Science, pages 337-340. Springer, 2008.

[23] K. Deb, A. Pratap, S. Agarwal, and T. Meyarivan. A fast elitist multi-objective genetic algorithm: Nsga-ii. IEEE Transactions on Evolutionary Computation, 6:182-197, 2000.

[24] S. Debray, P. López-García, M. V. Hermenegildo, and N.-W. Lin. Estimating the Computational Cost of Logic Programs. In Static Analysis Symposium, SAS'94, number 864 in LNCS, pages 255-265, Namur, Belgium, September 1994. Springer-Verlag.

[25] S. K. Debray and N. W. Lin. Cost Analysis of Logic Programs. ACM Transactions on Programming Languages and Systems, 15(5):826-875, November 1993.

[26] S. K. Debray, N.-W. Lin, and M. V. Hermenegildo. Task Granularity Analysis in Logic Programs. In Proc. 1990 ACM Conf. on Programming Language Design and Implementation (PLDI), pages 174-188. ACM Press, June 1990.

[27] S. K. Debray, P. López-García, M. V. Hermenegildo, and N.-W. Lin. Lower Bound Cost Estimation for Logic Programs. In 1997 International Logic Programming Symposium, pages 291-305. MIT Press, Cambridge, MA, October 1997. 
[28] K. Eder, J. Gallagher, P. Lopez-Garcia, H. Muller, Z. Banković, K. Georgiou, R. Haemmerlé, M. V. Hermenegildo, B. Kafle, S. Kerrison, M. Kirkeby, M. Klemen, X. Li, U. Liqat, J. Morse, M. Rhiger, and M. Rosendahl. ENTRA: Whole-Systems energy transparency. Microprocessors and Microsystems, 47, Part B:278-286, November 2016.

[29] C. Edwards. Lack of software support marks the low power scorecard at DAC. In Electronics Weekly (http://www.electronicsweekly.com)., volume 2472, pages 15-21, June 2011.

[30] B. Gaál. Multi-Level Genetic Algorithms and Expert System for Health Promotion. PhD thesis, Univ. of Panonia, Faculty of Information Technology, 12 2009.

[31] K. Georgiou, S. Kerrison, and K. Eder. On the Value and Limits of Multi-level Energy Consumption Static Analysis for Deeply Embedded Single and Multithreaded Programs, Oct. 2015. Technical report arXiv:1510.07095.

[32] M. Gerards, J. Hurink, P. Holzenspies, J. Kuper, and G. Smit. Analytic clock frequency selection for global dvfs. In Parallel, Distributed and Network-Based Processing (PDP), 2014 22nd Euromicro International Conference on, pages 512-519, Feb 2014.

[33] J. Giesl, T. Ströder, P. Schneider-Kamp, F. Emmes, and C. Fuhs. Symbolic evaluation graphs and term rewriting: a general methodology for analyzing logic programs. In PPDP, pages 1-12. ACM, 2012.

[34] S. Grebenshchikov, A. Gupta, N. P. Lopes, C. Popeea, and A. Rybalchenko. HSF(C): A Software Verifier Based on Horn Clauses - (Competition Contribution). In C. Flanagan and B. König, editors, TACAS, volume 7214 of LNCS, pages 549-551. Springer, 2012.

[35] N. Grech, K. Georgiou, J. Pallister, S. Kerrison, J. Morse, and K. Eder. Static analysis of energy consumption for LLVM IR programs. In Proceedings of the 18th International Workshop on Software and Compilers for Embedded Systems, SCOPES 2015, pages 12-21, New York, NY, USA, 2015. ACM.

[36] B. Grobauer. Cost recurrences for DML programs. In Proceedings of the Sixth ACM SIGPLAN International Conference on Functional Programming, ICFP '01, pages 253-264, New York, NY, USA, 2001. ACM.

[37] A. Gurfinkel, T. Kahsai, and J. A. Navas. Seahorn: A framework for verifying C programs (competition contribution). In Proc. of TACAS 2015, volume 9035 of $L N C S$, pages 447-450. Springer, 2015. 
[38] R. Haemmerlé, P. Lopez-Garcia, U. Liqat, M. Klemen, J. P. Gallagher, and M. V. Hermenegildo. A Transformational Approach to Parametric Accumulated-cost Static Profiling. In O. Kiselyov and A. King, editors, 13th International Symposium on Functional and Logic Programming (FLOPS 2016), volume 9613 of LNCS, pages 163-180. Springer, March 2016.

[39] K. S. Henriksen and J. P. Gallagher. Abstract Interpretation of PIC Programs through Logic Programming. In SCAM'06: Proceedings of the Sixth IEEE International Workshop on Source Code Analysis and Manipulation, pages 184196. IEEE Computer Society, 2006.

[40] M. V. Hermenegildo, F. Bueno, M. Carro, P. López, E. Mera, J. Morales, and G. Puebla. An Overview of Ciao and its Design Philosophy. Theory and Practice of Logic Programming, 12(1-2):219-252, January 2012. http://arxiv.org/abs/1102.5497.

[41] M. V. Hermenegildo, G. Puebla, and F. Bueno. Using Global Analysis, Partial Specifications, and an Extensible Assertion Language for Program Validation and Debugging. In K. R. Apt, V. Marek, M. Truszczynski, and D. S. Warren, editors, The Logic Programming Paradigm: a 25-Year Perspective, pages 161192. Springer-Verlag, July 1999.

[42] M. V. Hermenegildo, G. Puebla, F. Bueno, and P. Lopez-Garcia. Integrated Program Debugging, Verification, and Optimization Using Abstract Interpretation (and The Ciao System Preprocessor). Science of Computer Programming, 58(1-2):115-140, October 2005.

[43] C. Herrmann, A. Bonenfant, K. Hammond, S. Jost, H.-W. Loidl, and R. Pointon. Automatic Amortised Worst-Case Execution Time Analysis. In 7th International Workshop on Worst-Case Execution Time Analysis (WCET'07), volume 6 of OASIcs. Schloss Dagstuhl-Leibniz-Zentrum fuer Informatik, 2007.

[44] J. Hoffmann, K. Aehlig, and M. Hofmann. Multivariate amortized resource analysis. ACM Transactions on Programming Languages and Systems, 34(3):14:1-14:62, 2012.

[45] H. Hojjat, F. Konecný, F. Garnier, R. Iosif, V. Kuncak, and P. Rümmer. A Verification Toolkit for Numerical Transition Systems - Tool Paper. In D. Giannakopoulou and D. Méry, editors, FM 2012: Formal Methods - 18th International Symposium, Paris, France, August 27-31, 2012. Proceedings, volume 7436 of Lecture Notes in Computer Science, pages 247-251. Springer, 2012.

[46] A. Igarashi and N. Kobayashi. Resource usage analysis. In Proceedings of the 29th ACM SIGPLAN-SIGACT Symposium on Principles of Programming Languages, POPL '02, pages 331-342, New York, NY, USA, 2002. ACM. 
[47] R. Jayaseelan, T. Mitra, and X. Li. Estimating the worst-case energy consumption of embedded software. In IEEE Real Time Technology and Applications Symposium, pages 81-90. IEEE Computer Society, 2006.

[48] R. Jayaseelan, T. Mitra, and X. Li. Estimating the Worst-Case Energy Consumption of Embedded Software. In IEEE Real-Time and Embedded Technology and Applications Symposium (RTAS 2006), pages 81-90. IEEE Computer Society, 2006.

[49] R. Jejurikar, C. Pereira, and R. Gupta. Leakage aware dynamic voltage scaling for real-time embedded systems. In Proceedings of the 41st Annual Design Automation Conference, DAC '04, pages 275-280, New York, NY, USA, 2004. ACM.

[50] S. Kerrison and K. Eder. Measuring and modelling the energy consumption of multithreaded, multi-core embedded software. ICT Energy Letters, pages 1819, July 2014.

[51] S. Kerrison and K. Eder. Energy Modeling of Software for a Hardware Multithreaded Embedded Microprocessor. ACM Transactions on Embedded Computing Systems, 14(3):1-25, April 2015.

[52] V. Kianzad, S. Bhattacharyya, and G. Qu. Casper: an integrated energy-driven approach for task graph scheduling on distributed embedded systems. In 16th IEEE International Conference on Application-Specific Systems, Architecture Processors (ASAP'05), pages 191-197, 2005.

[53] P. Kumar and S. Palani. A dynamic voltage scaling with single power supply and varying speed factor for multiprocessor system using genetic algorithm. In 2012 International Conference on Pattern Recognition, Informatics and Medical Engineering (PRIME), pages 342-346. IEEE, 2012.

[54] S. Lafond and J. Lilius. Energy consumption analysis for two embedded java virtual machines. J. Syst. Archit., 53(5-6):328-337, 2007.

[55] C. Lattner and V. Adve. LLVM: A compilation framework for lifelong program analysis and transformation. In Proc. of the 2004 International Symposium on Code Generation and Optimization (CGO), pages 75-88. IEEE Computer Society, March 2004.

[56] J. Lee, T. Avgerinos, and D. Brumley. TIE: Principled Reverse Engineering of Types in Binary Programs. In Proceedings of the Network and Distributed System Security Symposium, NDSS 2011. The Internet Society, February 2011.

[57] Y. Lichtenstein and E. Y. Shapiro. Abstract algorithmic debugging. In R. A. Kowalski and K. A. Bowen, editors, Fifth International Conference and Symposium on Logic Programming, pages 512-531, Seattle, Washington, August 1988. MIT. 
[58] U. Liqat, Z. Banković, P. Lopez-Garcia, and M. V. Hermenegildo. Inferring Energy Bounds Statically by Evolutionary Analysis of Basic Blocks. In Workshop on High Performance Energy Efficient Embedded Systems (HIP3ES 2016), 2016. arXiv: 1601.02800.

[59] U. Liqat, Z. Banković, P. Lopez-Garcia, and M. V. Hermenegildo. An Evolutionary Scheduling Approach for Trading-off Accuracy vs. Verifiable Energy in Multicore Processors. Logic Journal of the IGPL, 25(6):1006-1019, December 2017.

[60] U. Liqat, Z. Banković, P. Lopez-Garcia, and M. V. Hermenegildo. Inferring Energy Bounds via Static Program Analysis and Evolutionary Modeling of Basic Blocks. In Pre-proceedings of the 27th International Symposium on LogicBased Program Synthesis and Transformation (LOPSTR'17), October 2017. arXiv:1601.02800.

[61] U. Liqat, K. Georgiou, S. Kerrison, P. Lopez-Garcia, M. V. Hermenegildo, J. P. Gallagher, and K. Eder. Inferring Parametric Energy Consumption Functions at Different Software Levels: ISA vs. LLVM IR. In M. V. Eekelen and U. D. Lago, editors, Foundational and Practical Aspects of Resource Analysis: 4th International Workshop, FOPARA 2015, London, UK, April 11, 2015. Revised Selected Papers, volume 9964 of Lecture Notes in Computer Science, pages 81-100. Springer, 2016.

[62] U. Liqat, S. Kerrison, A. Serrano, K. Georgiou, P. Lopez-Garcia, N. Grech, M. V. Hermenegildo, and K. Eder. Energy Consumption Analysis of Programs based on XMOS ISA-level Models. In G. Gupta and R. Peña, editors, LogicBased Program Synthesis and Transformation, 23rd International Symposium, LOPSTR 2013, Revised Selected Papers, volume 8901 of Lecture Notes in Computer Science, pages 72-90. Springer, 2014.

[63] P. López-García, L. Darmawan, and F. Bueno. A Framework for Verification and Debugging of Resource Usage Properties. In M. V. Hermenegildo and T. Schaub, editors, Technical Communications of the 26th Int'l. Conference on Logic Programming (ICLP'10), volume 7 of Leibniz International Proceedings in Informatics (LIPIcs), pages 104-113, Dagstuhl, Germany, July 2010. Schloss Dagstuhl-Leibniz-Zentrum fuer Informatik.

[64] P. Lopez-Garcia, L. Darmawan, F. Bueno, and M. V. Hermenegildo. IntervalBased Resource Usage Verification: Formalization and Prototype. In R. P. na, M. Eekelen, and O. Shkaravska, editors, Foundational and Practical Aspects of Resource Analysis. Second International Workshop FOPARA 2011, Revised Selected Papers, volume 7177 of Lecture Notes in Computer Science, pages 54-71. Springer-Verlag, 2012. 
[65] P. Lopez-Garcia, R. Haemmerlé, M. Klemen, U. Liqat, and M. V. Hermenegildo. Towards Energy Consumption Verification via Static Analysis. In Workshop on High Performance Energy Efficient Embedded Systems (HIP3ES), arXiv:1501.03064, 2015. arXiv:1512.09369.

[66] D. May. The XMOS XS1 architecture. available online: http://www.xmos.com/published/xmos-xs1-architecture, 2013.

[67] M. Méndez-Lojo, J. Navas, and M. Hermenegildo. A Flexible (C)LP-Based Approach to the Analysis of Object-Oriented Programs. In 17th International Symposium on Logic-based Program Synthesis and Transformation (LOPSTR 2007), number 4915 in Lecture Notes in Computer Science, pages 154-168. Springer-Verlag, August 2007.

[68] E. Mera, P. López-García, M. Carro, and M. V. Hermenegildo. Towards Execution Time Estimation in Abstract Machine-Based Languages. In 10th Int'l. ACM SIGPLAN Symposium on Principles and Practice of Declarative Programming (PPDP'08), pages 174-184. ACM Press, July 2008.

[69] E. Mera, T. Trigo, P. López-García, and M. V. Hermenegildo. Profiling for RunTime Checking of Computational Properties and Performance Debugging. In Practical Aspects of Declarative Languages (PADL'11), volume 6539 of Lecture Notes in Computer Science, pages 38-53. Springer-Verlag, January 2011.

[70] M. Mezmaz, Y. C. Lee, N. Melab, E. Talbi, and A. Zomaya. A bi-objective hybrid genetic algorithm to minimize energy consumption and makespan for precedence-constrained applications using dynamic voltage scaling. In IEEE Congress on Evolutionary Computation (CEC'10), pages 1-8. IEEE, 2010.

[71] M.-S. Mezmaz, Y. Kessaci, Y. Lee, N. Melab, E.-G. Talbi, A. Zomaya, and D. Tuyttens. A parallel island-based hybrid genetic algorithm for precedenceconstrained applications to minimize energy consumption and makespan. In Grid Computing (GRID), 2010 11th IEEE/ACM International Conference on, pages 274-281, Oct 2010.

[72] A. Miyoshi, C. Lefurgy, E. Van Hensbergen, R. Rajamony, and R. Rajkumar. Critical power slope: Understanding the runtime effects of frequency scaling. In Proceedings of the 16th International Conference on Supercomputing, ICS '02, pages 35-44, New York, NY, USA, 2002. ACM.

[73] R. G. Morgan and S. A. Jarvis. Profiling Large-Scale Lazy Functional Programs. Journal of Functional Programing, 8(3):201-237, 1998.

[74] K. Muthukumar and M. Hermenegildo. Compile-time Derivation of Variable Dependency Using Abstract Interpretation. Journal of Logic Programming, 13(2/3):315-347, July 1992. 
[75] J. Navas, M. Méndez-Lojo, and M. Hermenegildo. Safe Upper-bounds Inference of Energy Consumption for Java Bytecode Applications. In The Sixth NASA Langley Formal Methods Workshop (LFM 08), pages 29-32, April 2008. Extended Abstract.

[76] J. Navas, M. Méndez-Lojo, and M. V. Hermenegildo. User-Definable Resource Usage Bounds Analysis for Java Bytecode. In Proceedings of the Workshop on Bytecode Semantics, Verification, Analysis and Transformation (BYTECODE'09), volume 253 of Electronic Notes in Theoretical Computer Science, pages 65-82. Elsevier - North Holland, March 2009.

[77] J. Navas, E. Mera, P. López-García, and M. Hermenegildo. User-Definable Resource Bounds Analysis for Logic Programs. In 23rd International Conference on Logic Programming (ICLP'07), volume 4670 of Lecture Notes in Computer Science. Springer, 2007.

[78] F. Nielson, H. Nielson, and C. Hankin. Principles of Program Analysis. Springer-Verlag, 1999.

[79] F. Nielson, H. Nielson, and H. Seidl. Automatic complexity analysis. In Programming Languages and Systems, volume 2305 of Lecture Notes in Computer Science, pages 243-261. Springer Berlin Heidelberg, 2002.

[80] J. Pallister, S. Kerrison, J. Morse, and K. Eder. Data Dependent Energy Modeling for Worst Case Energy Consumption Analysis. In S. Stuijk, editor, Proc. of the 20th International Workshop on Software and Compilers for Embedded Systems (SCOPES 2017), pages 51-59. ACM, 2017.

[81] F. Paterna, A. Acquaviva, A. Caprara, F. Papariello, G. Desoli, and L. Benini. An efficient on-line task allocation algorithm for qos and energy efficiency in multicore multimedia platforms. In Design, Automation Test in Europe Conference Exhibition (DATE), 2011, pages 1-6, March.

[82] G. Puebla, F. Bueno, and M. V. Hermenegildo. An Assertion Language for Constraint Logic Programs. In P. Deransart, M. V. Hermenegildo, and J. Maluszynski, editors, Analysis and Visualization Tools for Constraint Programming, number 1870 in LNCS, pages 23-61. Springer-Verlag, September 2000.

[83] G. Puebla and M. V. Hermenegildo. Optimized Algorithms for the Incremental Analysis of Logic Programs. In International Static Analysis Symposium (SAS 1996), number 1145 in Lecture Notes in Computer Science, pages 270-284. Springer-Verlag, September 1996.

[84] M. Rosendahl. Automatic Complexity Analysis. In 4th ACM Conference on Functional Programming Languages and Computer Architecture (FPCA'89), pages 144-156. ACM Press, 1989. 
[85] P. M. Sansom and S. L. P. Jones. Time and Space Profiling for Non-Strict, Higher-Order Functional Languages. In R. K. Cytron and P. Lee, editors, Proceedings of the 22nd ACM SIGPLAN-SIGACT Symposium on Principles of Programming Languages, POPL'95, pages 355-366, New York, NY, USA, 1995. ACM.

[86] A. Serrano, P. Lopez-Garcia, F. Bueno, and M. V. Hermenegildo. Sized Type Analysis for Logic Programs (technical communication). In T. Swift and E. Lamma, editors, Theory and Practice of Logic Programming, 29th Int'l. Conference on Logic Programming (ICLP'13) Special Issue, On-line Supplement, volume 13, pages 1-14. Cambridge U. Press, August 2013.

[87] A. Serrano, P. Lopez-Garcia, and M. V. Hermenegildo. Resource Usage Analysis of Logic Programs via Abstract Interpretation Using Sized Types. Theory and Practice of Logic Programming, 30th Int'l. Conference on Logic Programming (ICLP'14) Special Issue, 14(4-5):739-754, 2014.

[88] S. A. Seshia and J. Kotker. Gametime: A toolkit for timing analysis of software. In P. A. Abdulla and K. R. M. Leino, editors, TACAS, volume 6605 of Lecture Notes in Computer Science, pages 388-392. Springer, 2011.

[89] S. Sidiroglou-Douskos, S. Misailovic, H. Hoffmann, and M. Rinard. Managing performance vs. accuracy trade-offs with loop perforation. In Proceedings of the 19th ACM SIGSOFT symposium and the 13th European conference on Foundations of software engineering, pages 124-134. ACM, 2011.

[90] V. Tiwari, S. Malik, and A. Wolfe. Power Analysis of Embedded Software: a First Step Towards Software Power Minimization. IEEE Trans. VLSI Syst., 2(4):437-445, 1994.

[91] V. Tiwari, S. Malik, A. Wolfe, and M. T. C. Lee. Instruction level power analysis and optimization of software. In Proceedings of VLSI Design, pages 326-328, 1996.

[92] P. Vasconcelos and K. Hammond. Inferring Cost Equations for Recursive, Polymorphic and Higher-Order Functional Programs. In Proceedings of the International Workshop on Implementation of Functional Languages, volume 3145 of Lecture Notes in Computer Science, pages 86-101. Springer-Verlag, Sept. 2003.

[93] C. Vaucheret and F. Bueno. More Precise yet Efficient Type Inference for Logic Programs. In 9th International Static Analysis Symposium (SAS'02), volume 2477 of Lecture Notes in Computer Science, pages 102-116. Springer-Verlag, September 2002.

[94] P. Wagemann, T. Distler, T. Honig, H. Janker, R. Kapitza, and W. SchroderPreikschat. Worst-case energy consumption analysis for energy-constrained 
embedded systems. In Real-Time Systems (ECRTS), 2015 27th Euromicro Conference on, pages 105-114, July 2015.

[95] D. Watt. Programming XC on XMOS Devices. XMOS Limited, 2009.

[96] B. Wegbreit. Mechanical Program Analysis. Communications of the ACM, 18(9):528-539, September 1975.

[97] R. Wilhelm, J. Engblom, A. Ermedahl, N. Holsti, S. Thesing, D. Whalley, G. Bernat, C. Ferdinand, R. Heckmann, T. Mitra, F. Mueller, I. Puaut, P. Puschner, J. Staschulat, and P. Stenström. The worst-case execution-time problem - Overview of methods and survey of tools. ACM Trans. Embedded Comput. Syst., 7(3), 2008.

[98] XMOS. Use xTIMEcomposer and XSCOPE to trace data in real-time, 2013. Available online at: https://www.xmos.com/download/public/Trace-data-withXScope(X9923H).pdf.

[99] XMOS. xTAG v3.0 Hardware Manual, June 2015. Available online at: https://www.xmos.com/download/private/xTAG-3-Hardware-Manual

[100] XMOS-Ltd. XS1-SU01A-FB96 Datasheet, November 2012.

[101] L. Xu, F. Sun, and Z. Su. Constructing Precise Control Flow Graphs from Binaries. University of California, Davis, Tech. Rep, 2009.

[102] F. Yao, A. Demers, and S. Shenker. A scheduling model for reduced cpu energy. 2013 IEEE 54th Annual Symposium on Foundations of Computer Science, 0:374, 1995.

[103] S. Yassa, R. Chelouah, and B. Granado. Multi-objective approach for energyaware workflow scheduling in cloud computing environments. The Scientific World Journal, 2013. Article ID: 350934.

[104] G. Ye, R. Rao, and M. Li. A multiobjective resources scheduling approach based on genetic algorithms in grid environment. In Fifth International Conference on Grid and Cooperative Computing Workshops, 2006. GCCW'06, pages 504509. IEEE, Oct 2006.

[105] J. Yu, M. Kirley, and R. Buyya. Multi-objective planning for workflow execution on grids. In Proceedings of the 8th IEEE/ACM International Conference on Grid Computing, GRID '07, pages 10-17, Washington, DC, USA, 2007. IEEE Computer Society.

[106] F. Zuleger, S. Gulwani, M. Sinn, and H. Veith. Bound analysis of imperative programs with the size-change abstraction (extended version). CoRR, abs/1203.5303, 2012. 